\title{
Traditional knowledge in semi-rural close to industrial areas: ethnobotanical studies in western Gironès (Catalonia, Iberian Peninsula)
}

\author{
Airy Gras ${ }^{1,2,3}$, Ginesta Serrasolses ${ }^{1}$, Joan Vallès ${ }^{1,3,4}$ and Teresa Garnatje $2^{2^{*}}$
}

\begin{abstract}
Background: The western Gironès is a district located in NE Catalonia (NE Iberian Peninsula). This area comprising $186.55 \mathrm{~km}^{2}$ and 10,659 inhabitants is composed of 5 municipalities encompassing 29 villages, located in the hydrographic basins of the Ter and Llémena rivers.

Methods: Following the methodology based on the semi-structured interviews, we carried out 40 interviews with 57 informants, 31 were women and the remaining 26 were men, with an average age of 78.6 years.

Results: In the present study, data from 316 taxa (301 angiosperms, 8 gymnosperms, and 7 pteridophytes) belonging to 89 botanical families were collected. The interviewed informants referred 3776 UR of 298 taxa, 1933 $(51.19 \%)$ of them corresponding to the food category, 949 (25.13\%) to the medicinal ones, and 894 (23.68\%) to other uses. In addition, 581 vernacular names for 306 species, subspecies, and varieties have also been collected.

Conclusions: These results reveal the validity of traditional knowledge in the studied area, which can be seriously threatened by the loss of its rural condition and its proximity to industrialized areas.
\end{abstract}

Keywords: Ethnobotany, Ethnoflora, Gironès, Medicinal uses, Plant uses, Traditional knowledge

\section{Introduction}

The Catalan-speaking territories constitute a cultural unity that has attracted the interest of researchers from various disciplines. Since two pioneering $\mathrm{PhD}$ theses $[1$, 2], several similar studies have been devoted to ethnobotanical research in these areas ([3-14], among others), and papers derived from these academic works) with the common objective of collecting, inventorying, preserving, and disseminating the popular uses of plants. The so-called acculturation process taking place in the industrialized areas, in other words the adoption of modern culture to the detriment of the traditional one [15], is the main cause of the loss of this knowledge, which must be available for future generations.

\footnotetext{
* Correspondence: tgarnatje@ibb.csic.es

${ }^{2}$ Institut Botànic de Barcelona (IBB, CSIC-ICUB), Passeig del Migdia s/n, Parc de Montjuïc, 08038 Barcelona, Catalonia, Spain

Full list of author information is available at the end of the article
}

For this reason, the research that was initially focused on non-industrialized areas [16-22] has now been expanded in industrialized areas due to their rapid loss of traditional knowledge [23-27].

Although ethnobotany, as defined by Harshberger [28], was conceived to study the plants used by a particular human group-not limited to any type of usemost studies have placed special interest in medicinal plants [29-31] and secondly in those used for food purposes [32-36]. The studies comprising the whole ethnobotanical knowledge of an industrialized area are less frequent. This situation is explained, according to Gras et al. [37], due to the fact that medicinal and food uses are most related to human health, which is still valid despite the above-mentioned acculturation process. In addition, according to these authors, plants with medicinal and food uses are more susceptible to being potentially used or transformed into commercial products.

(c) The Author(s). 2019 Open Access This article is distributed under the terms of the Creative Commons Attribution 4.0 International License (http://creativecommons.org/licenses/by/4.0/), which permits unrestricted use, distribution, and 


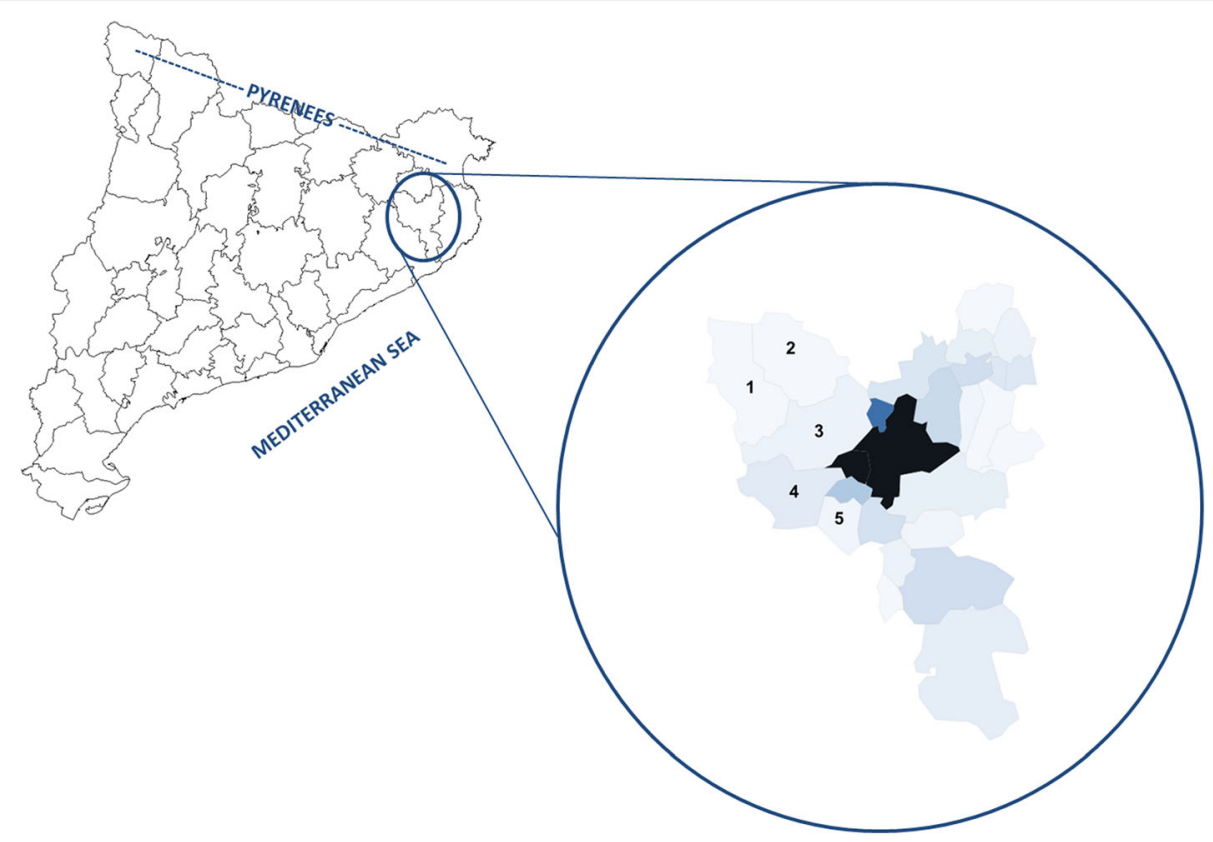

Fig. 1 Location of the studied areas. In blue, Gironès district, including the study area (western Gironès). The figures correspond to the municipalities studied. 1: Sant Martí de Llémena, 2: Canet d'Adri, 3: Sant Gregori, 4: Bescanó, and 5: Aiguaviva. The intensity of colors is related to population density

The district (in Catalan "comarca") of Gironès is located in NE Catalonia (Fig. 1), in its turn situated in the NE Iberian Peninsula. Our study was centered on the western part of this district, considering the natural unit constituted by territories under the influence of the hydrographic basins of the Ter and Llémena rivers. The western Gironès is composed of 5 municipalities encompassing 29 villages. The study area comprises $186.55 \mathrm{~km}^{2}$ and 10,659 inhabitants [38] representing a density of 57.14 inhabitants $/ \mathrm{km}^{2}$. The altitudes range from $102 \mathrm{~m}$ a.s.l. in the locality of Bescanó to $256 \mathrm{~m}$ a.s.l. in Sant Martí de Llémena.

The Gironès district has a Mediterranean climate with an irregular rain distribution with relatively wet springs and autumns and dry summers and winters. The mean rainfall increases in SE-NW direction, with values around $1000 \mathrm{~mm}$ per year in the North-Western edge. Winters are moderately cold and summers are hot, with an annual mean of $14.4{ }^{\circ} \mathrm{C}$ [39].

The landscape of the area was described by Girbal [40] and is very heterogeneous, the low areas are occupied by dry lands, with herbal communities from the alliances Diplotaxion erucoidis and Secalion cerealis. In the mountainous regions, there is an altitudinal gradient, from the calcicolous scrubs of Rosmarino-Ericion with Pinus halepensis. in the lowlands to the beeches with Pyrenean squill (Scillo liliohyacinthi-Fagetum sylvaticae) in the highlands. The intermediate zones are occupied by holm oak forests (Viburno tini-Quercetum ilicis subass. pistacietosum and
Asplenio-Quercetum ilicis) and by a narrow belt of oak (Quercus pubescens) in the upper part connecting with beech (Fagus sylvatica).

Economically, this area has evolved through different historical periods: prior to the industrial era, it was based on agriculture-mainly cereals-livestock, and forest management for timber and charcoal production and a second period based on textile industry. Currently, these villages do not have their own economy and they have become dormitories for people working in Girona, the capital of the district, with an important economic activity [38]. To sum up, western Gironès is still at least what can be called a semi-rural area, since agriculture is still alive there, but three of its municipalities (Aiguaviva, Bescanó, Sant Gregori) play the above-mentioned role of dormitory to the close metropolitan, industrial area. Additionally, the river Llémena valley hosts an important number of secondary residences for people from the neighboring territory, especially from Girona, the 11th biggest city in Catalonia, with a population very close to 100,000 , and head of one of the four Catalonian administrative units (province) including several districts, as among which the one here considered [38].

The main goals of the present study were (i) to collect plant uses and their vernacular names in a semi-rural area, to inventory and preserve this knowledge in order for it to be available to future generations, and (ii) to analyze the obtained results in order to establish some comparisons with similar territories. 


\section{Material and methods}

\section{Field work}

The fieldwork took place from June 2013 to August 2014. We carried out 40 interviews to 57 informants: 23 were individual and 17 concerned 2 people, no one implying a bigger group. Out of the interviewed people, 31 (54\%) were women and the remaining 26 (46\%) were men. The methodology used was based on the semi-structured interviews [41] avoiding closed questionnaires and direct questions that could have an implicit answer so as not to coerce informants' answers (Fig. 2). Conversations were developed in the Catalan language, common to interviewers and interviewees. During the ethnobotanical surveys, we not only focused on medicinal and food uses but also asked for knowledge of plants with other uses. The popular names of plants, in Catalan, were also collected.

We have recorded information on both wild and cultivated plants, and also on plants that can be bought through commerce. Results are presented according to the classification of the folk uses of the species in three main categories: medicinal, food, and other uses. Within the food category, we distinguish the human and animal uses. To define the types of medicinal plant uses, we basically follow Cook's Economic Botany Data Collection Standard [42].
The plant taxa cited by the informants were identified using the Flora dels Països Catalans [43] and the Flora Manual dels Països Catalans [44], which we basically follow for nomenclature. The allocation of families has been done following the APG IV [45]. The herbarium vouchers have been deposited in the herbarium $\mathrm{BCN}$ (Centre de Documentació de Biodiversitat Vegetal, Universitat de Barcelona).

The field work respected the ethical principles of the International Society of Ethnobiology [46] and we had the prior oral informed consent of the informants [47].

\section{Data analysis}

The interviews were recorded and subsequently transcribed, and all the information obtained was entered into the database of our research group (www.etnobotanica.cat). The analyses were carried out with Excel (Microsoft Excel 2007) and XLSTAT (v2007.5, Addinsoft SARL) programs. To analyze the results, we have used the use report (hereinafter, UR) [48].

With the aim of assessing the state of knowledge, studies of quantitative ethnobotany were also performed and the following indices were calculated: ethnobotanicity index (EI; [49]), which is the quotient between the number of plants used and the total number of plants that constitute the flora of the territory, expressed as a
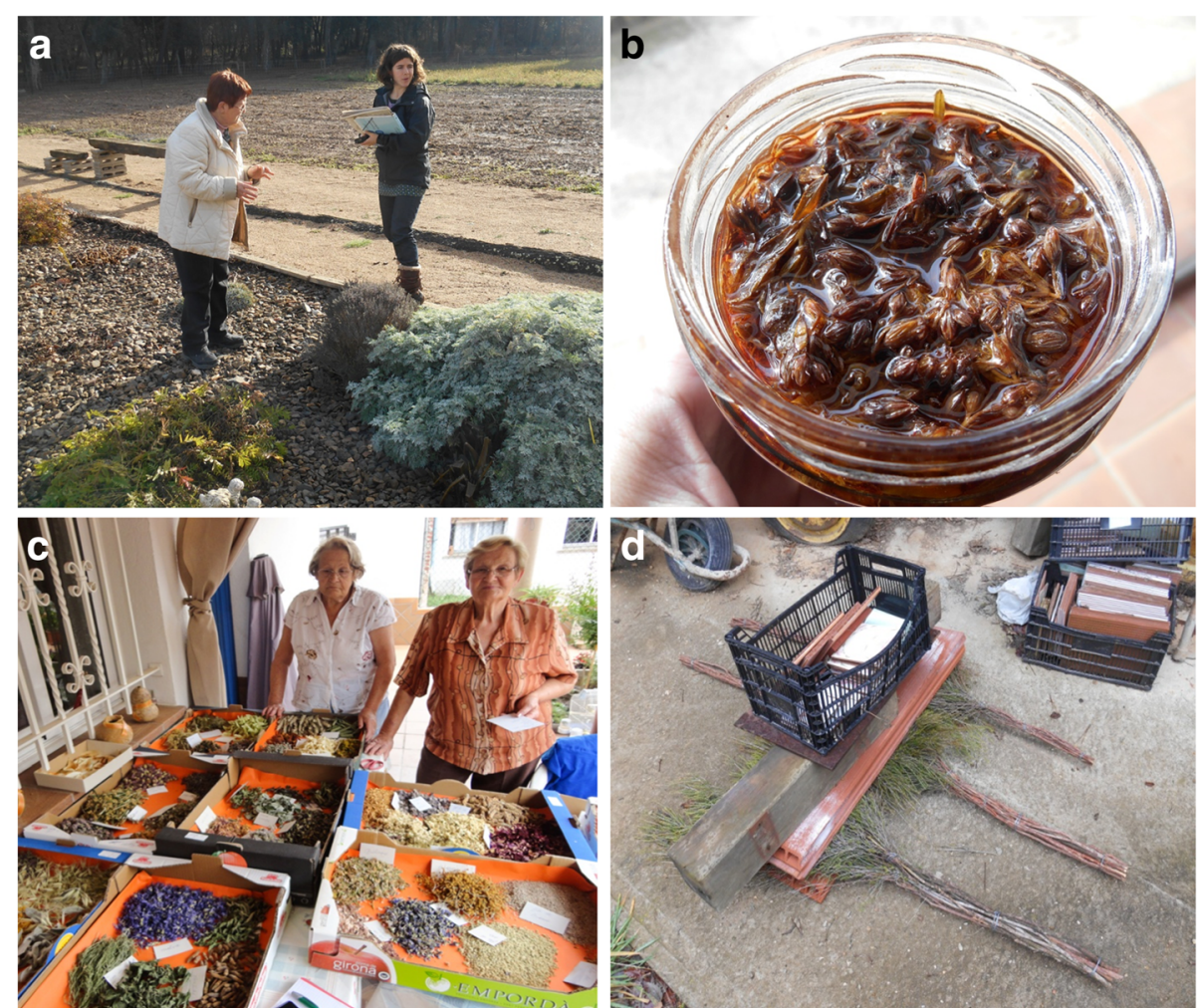

Fig. 2 Exemples of ethnobotanical interviews and some products derived from plants. a Interview in a homegarden. b Hypericum perforatum oil for burns and bumps. c Informants with ingredients to prepare ratafia. d Erica scoparia brooms 
percentage; the informant consensus factor ( $\mathrm{F}_{\mathrm{IC}}$; [50]), which is the quotient between the number of medicinal use reports minus the number of used medicinal plants and the number of medicinal use reports minus one. This indicates the degree of reliability of the uses claimed (higher when closer to 1).

Number of medicinal plants used per informant $(\mathrm{P} / \mathrm{I})$, per inhabitant $(\mathrm{P} / \mathrm{H})$, and per unit of area $\left(\mathrm{P} / \mathrm{km}^{2}\right)$ were calculated, in order to compare with other territories from which this information is provided only for this kind of useful plants. The linguistic diversity index [51], obtained by dividing the number of folk names by the number of taxa reported, has been calculated to illustrate the cultural richness of the folk plant knowledge.

Finally, we calculated the recently proposed index of taxon usefulness in mixtures (ITUM; [52]), which is the quotient between the number of citation of this taxon in mixtures and its total citations, whether with simple or complex presentation. This index indicates the exclusiveness of taxa in mixtures when the value is one or closer to one.

\section{Results and discussion}

This study contributes information to complete the ethnobotanical knowledge in the North Eastern Catalan linguistic and cultural area, where still a territory is to be investigated before being able to perform a meta-analytic work. It also enlarges the ethnofloristic knowledge of the Iberian territories, which are among the most studied in Europe [27]. We believe that, in general, increasing data on Catalan and Iberian folk plant knowledge provides them with a bigger robustness, apart from contributing new or rare uses and taxa used. Plants having appeared not very long time ago in European folk phytotherapy constitute not the only but a good example of such additions that prospects as the present one can bring to the ethnoflora. Although when first contacting the informants we indicate that we are interested in orally-transmitted traditional uses, in some cases, they report to us that a certain knowledge on a plant use is recently acquired. Just as a case example, Aloe vera does not appear in the pioneering works on Catalan ethnobotany $[1,2]$, but is importantly present, with ten use reports, in this one. Even if these data may have not been considered in some occasions, a reflection should be initiated on the new incorporations to folk knowledge, which will become tradition and will lead to a renewed paradigm in plant uses.

\section{Characteristics of the interviewees}

The average age of the informants is 78.6 years, ranging from 58 to 92 , the interval between 78 and 80 years being the one that accumulates a greater number of informants. This average is one of the highest values found in the recent studies carried out in similar areas and only surpassed in the island of Formentera [11].

Most informants were native (74\%) and the remaining ones have lived in the area for more than half of their lives. Only $10 \%$ are native from the neighboring district of la Selva.

Regarding their work, most of the men have been farmers $(18 \%)$ or shepherds (5\%), while most of the women have combined the farm work with household affairs (23\%). Other professions linked to the territory are textile (16\%) and hotel (7\%) industries, both important economic activities in this area.

\section{Plant species, use reports, and botanical families}

Data from 316 taxa (301 angiosperms, 8 gymnosperms, and 7 pteridophytes) belonging to 89 botanical families were collected in the present study. Thirteen taxa have only been determined at generic level and 19 present infraspecific categories. In the first case, taxa-in fact ethnotaxa-were referred to by the informants without specific category. It could be due to several or all species of the genus being used, or to the fact that they were not able to distinguish the taxa. The complete catalog of the recorded useful plants in the studied area is contained in Serrasolses [13], and the data concerning all plants, shown later, are synthesized, arranged by large use categories.

The five best represented families are Lamiaceae (12.39\%), Poaceae (9.25\%), Rosaceae (7.35\%), Asteraceae (6.84\%), and Fabaceae (5.55\%), which partially coincides with the findings in other territories with similar characteristics $[6,10,12,53]$ and at the same time represents the most common botanical families, apart from Apiaceae and Rutaceae, of the Mediterranean flora [44]. This fact links with the idea that the closer to civilization a plant grows, the more it is used by local people [54-57].

The interviewed informants refer 3776 UR of 298 taxa, $1933(51.19 \%)$ of them corresponding to the food category, 949 (25.13\%) to the medicinal ones, and 894 $(23.68 \%)$ to other uses. The mean of UR per informant is 66.25 , and 5.23 taxa per informant are cited, but these values show very large deviations due to the differences in knowledge that exist between the informants.

Medicinal plants are the most reported in the majority of ethnobotanical works carried out in the Catalan Countries $[6,10,58]$. However, in the present study, food uses are the most cited by the informants due to the collection of a large number of recipes devoted to the preparation of ratafia (see comments on this beverage in 3.5). This traditional Catalan liqueur [59], prepared with the immature fruit of Juglans regia and numerous species of preferably aromatic plants, is still consumed in areas close to the study area [60]. 


\section{Quantitative ethnobotany}

Some quantitative ethnobotany indexes concerning ten territories (the one here studied included) of the Catalan linguistic area are presented in Table 1 . The ethnobotanicity index, not having into account the 50 taxa of allochthonous plants recorded, is $22.56 \%$ for the studied area; this roughly meaning that between one-fifth and one-quarter of the plants of the area have been claimed as useful by the informants. It occupies an intermediate position in the range of the values obtained for other Catalan-language studied areas The informant consensus factor $\left(\mathrm{F}_{\mathrm{IC}}\right)$ of medicinal information obtained for our interviewees $(0.86)$ is close to the highest values in the quoted areas. Interestingly, this value, accounting for the consistency (thus, reliability) of plant use within a cultural and geographical group, which is an indicative of a generationally transmitted knowledge is higher to those obtained in Mexican areas $(0.75,0.79 ;[61,62])$. Recently, an ethnobotanical study of medicinal foods used by practitioners in an Indian area shows $\mathrm{F}_{\mathrm{IC}}$ for the different ailments treated ranging from 0 to 1 , but low in mean value $(0.26 ;[63])$. The results are similar (with a highest value of 0.72 ) in a study of medicinal plants in the Greek Aegean Islands [64]. This indicates that the traditional pool of knowledge on plant use and management is still alive in the studied area. Consequently, we can state that there is a high consistency in folk plant knowledge in the industrial European zone considered, where it could have been hypothesized it would be lower, even as compared with less industrialized Asian or American territories, where ethnobiological data are a priori supposed to be high, robust, and less eroded.

\section{Medicinal uses}

Our informants mentioned 137 species with medicinal uses and 949 use reports, $81.66 \%$ of which are referring to human medicine, $7.06 \%$ to veterinary, and $1.37 \%$ to both human and veterinary medicines (Table 2). No information was reported for the remaining 9.91\%. The mean of medicinal taxa cited by informant is 2.40 . This number of medicinal plants, quoted by the 57 informants, is close (slightly lower in ratio taxa/informant) to the one found in an area covering a part of the island of Mallorca, with a comparable number of interviewees as well: 121 taxa quoted by 42 informants [57]. Conversely, a recent study in a Turkish region [65] reports 92 taxa (35\% of which with medicinal uses) quoted by 123 informants, i.e., a clearly lower ratio. Similarly, a research in a Myanmar area [66] records 75 medicinal taxa cited by 206 informants. This is also the case in Europe: in the Greek Aegean Islands, 200 informants reported uses of 109 medicinal plants [64]; the authors state that these plants are used, but they do not mention any other plant quoted by the informants and not currently used. This reinforces the above-exposed argument that the ethnobotanical corpus is still relevant in industrialized areas, even in comparison with non- or less-industrialized territories, where the weight of this knowledge is a priori supposed to be higher. There is still time left (probably in its very end) to collect the traditional knowledge on plant uses in industrialized zones, which is basic in order to reintroduce it to the younger generations, or to use certain information to develop a new useful product of higher reach.

The 20 most cited species are included in Table 3. Sambucus nigra and Thymus vulgaris, with 81 and 78 UR, respectively, are the species heading the ranking. These taxa are among the most cited in other Catalan territories $[6,10,12,53,58]$. Concerning the families, Lamiaceae (164 UR; 17.28\%) and Adoxaceae (81 UR; $8.54 \%$ ) are the most reported ones followed by Asteraceae (79 UR; 8.32\%), Rutaceae (48 UR; 5.06\%), and Oleaceae (41 UR; 4.32\%). Lamiaceae and Asteraceae have a high number of representatives in the Mediterranean flora and Rutaceae include the citrus fruit species, whereas Adoxaceae is among the most cited families

Table 1 Quantitative ethnobotany indexes in ten territories (in italic, the one here studied) in the Catalan linguistic area. El: ethnobotanicity index; $F_{I C}$ : informant consensus factor; MP: number of medicinal plants

\begin{tabular}{|c|c|c|c|c|c|}
\hline Territory & $\mathrm{El}$ & $F_{1 C}$ & MP/informant & MP/inhabitant & $\mathrm{MP} / \mathrm{km}^{2}$ \\
\hline Alt Empordà [10] & 25.90 & 0.91 & 1.88 & $0.28 \times 10^{-2}$ & 0.25 \\
\hline Castelló [1] & 15.00 & - & 2.34 & $0.06 \times 10^{-2}$ & 0.06 \\
\hline Cerdanya $[2,7]$ & - & 0.93 & 1.11 & $0.82 \times 10^{-2}$ & 0.23 \\
\hline Segarra [76] & - & - & 3.17 & $0.54 \times 10^{-2}$ & 0.13 \\
\hline Eastern Mallorca [57] & 15.51 & 0.71 & 2.88 & $0.38 \times 10^{-2}$ & 0.51 \\
\hline Western Gironès (this paper) & 22.56 & 0.86 & 2.40 & $1.29 \times 10^{-2}$ & 0.73 \\
\hline Guilleries [4] & 20.00 & - & 5.64 & $0.58 \times 10^{-2}$ & 0.27 \\
\hline Montseny [6] & 23.20 & 0.91 & 1.95 & $0.44 \times 10^{-2}$ & 0.42 \\
\hline Pallars Jussà and Pallars Sobirà [5] & 29.10 & 0.87 & 1.66 & $2.32 \times 10^{-2}$ & 0.16 \\
\hline Ripollès [58] & 28.60 & 0.96 & 1.73 & $1.10 \times 10^{-2}$ & 0.29 \\
\hline
\end{tabular}


Table 2 Medicinal plants reported in the studied area

\begin{tabular}{|c|c|c|c|c|c|c|}
\hline Family & Taxon (voucher) & Catalan vernacular names & Medicinal use & Part used & Pharmaceutical form & $\overline{U R}$ \\
\hline Adoxaceae & $\begin{array}{l}\text { Sambucus nigra L. } \\
\text { (BCN113595) }\end{array}$ & Sabuquer. Saüc. Saüquer & $\begin{array}{l}\text { Anticatarrhal. antidiarrhoeal. } \\
\text { anti-inflammatory. antipneumo- } \\
\text { nic. antipyretic. buccal antisep- } \\
\text { tic. external antiseptic. for } \\
\text { amygdalitis. for earache. for } \\
\text { headache. emmenagogue. ex- } \\
\text { pectorant. not reported. ocular } \\
\text { antiseptic, refrigerant. stomachic }\end{array}$ & $\begin{array}{l}\text { Fruit. } \\
\text { inflorescence. } \\
\text { not reported }\end{array}$ & $\begin{array}{l}\text { Aerosol. bath. essence. } \\
\text { eyedrops. fumigation. } \\
\text { medicinal wine. not } \\
\text { reported. poultice. } \\
\text { syrup. tisane }\end{array}$ & 81 \\
\hline Amaranthaceae & $\begin{array}{l}\text { Beta vulgaris L. } \\
\text { subsp. vulgaris } \\
\text { var. crassa (Alef.) } \\
\text { Helm (BCN50761) }\end{array}$ & $\begin{array}{l}\text { Bleda. Polpa (elaborated } \\
\text { product). Remolatxa. Sucre } \\
\text { (elaborated product). Sucre } \\
\text { candi (elaborated product) }\end{array}$ & $\begin{array}{l}\text { Against taeniasis. anticatarrhal. } \\
\text { antihelminthic. expectorant }\end{array}$ & Root & $\begin{array}{l}\text { Decoction. direct use. } \\
\text { ointment }\end{array}$ & 8 \\
\hline \multirow[t]{2}{*}{ Amaryllidaceae } & $\begin{array}{l}\text { Allium cepa L. } \\
\text { (BCN28655) }\end{array}$ & Ceba & $\begin{array}{l}\text { Antitussive. expectorant. for } \\
\text { aphonia. not reported. } \\
\text { resolutive }\end{array}$ & Bulb & $\begin{array}{l}\text { Direct use. gargle. } \\
\text { poultice }\end{array}$ & 7 \\
\hline & $\begin{array}{l}\text { Allium sativum L. } \\
\text { (BCN29832) }\end{array}$ & All & $\begin{array}{l}\text { Analgesic. callicide. for earache. } \\
\text { hematocathartic }\end{array}$ & Bulb & $\begin{array}{l}\text { Alcoholic tincture. } \\
\text { direct use. embrocation }\end{array}$ & 29 \\
\hline Anacardiaceae & $\begin{array}{l}\text { Pistacia lentiscus } \\
\text { L. (BCN29907) }\end{array}$ & Llentiscle & Analgesic. teeth strengthening & $\begin{array}{l}\text { Aerial part. } \\
\text { not reported }\end{array}$ & Collutorium & 2 \\
\hline \multirow[t]{4}{*}{ Apiaceae } & $\begin{array}{l}\text { Conium } \\
\text { maculatum L. } \\
\text { (BCN32171) }\end{array}$ & Cicuta & Anticolitic & Aerial part & Bath & 1 \\
\hline & $\begin{array}{l}\text { Eryngium } \\
\text { campestre L. } \\
\text { (BCN31274) }\end{array}$ & Espinacal & $\begin{array}{l}\text { Anticholesterolemic. buccal } \\
\text { antiseptic }\end{array}$ & $\begin{array}{l}\text { Aerial part. } \\
\text { root }\end{array}$ & Collutorium. tisane & 5 \\
\hline & $\begin{array}{l}\text { Foeniculum } \\
\text { vulgare Mill. } \\
\text { (BCN26350) }\end{array}$ & Fonoll & $\begin{array}{l}\text { Anticolitic. antidiarrhoeal. } \\
\text { digestive. galactogene }{ }^{a} \text {. internal } \\
\text { antiseptic } c^{\mathrm{a}} \text { laxative. postpartum } \\
\text { coadjuvant }^{\mathrm{a}} \text {. stomachic }{ }^{\mathrm{b}}\end{array}$ & Aerial part & $\begin{array}{l}\text { Direct use. emulsion. } \\
\text { tisane }\end{array}$ & 15 \\
\hline & $\begin{array}{l}\text { Petroselinum } \\
\text { crispum (Mill.) Hill } \\
\text { (BCN29905) }\end{array}$ & Julivert & Abortive. hypoglycaemic & $\begin{array}{l}\text { Aerial part. } \\
\text { stem }\end{array}$ & Direct use & 2 \\
\hline Araceae & $\begin{array}{l}\text { Arum italicum } \\
\text { Mill. (BCN32358) }\end{array}$ & Xàrria. Xèrria & $\begin{array}{l}\text { Against tinea }^{a} \text {. } \\
\text { antihaemorrhoidal. anti- } \\
\text { inflammatory. antipyrotic }{ }^{b} \text {. for } \\
\text { amygdalitis. for skin disorders }^{a}\end{array}$ & Bulb. fruit & $\begin{array}{l}\text { Embrocation. not } \\
\text { reported. ointment }\end{array}$ & 6 \\
\hline Araliaceae & $\begin{array}{l}\text { Hedera helix L. } \\
\text { (BCN29869) }\end{array}$ & Heura. Heura d'alzina & Antihypertensive. antipyrotic & Leaf & Poultice. tisane & 3 \\
\hline \multirow[t]{2}{*}{ Asparagaceae } & $\begin{array}{l}\text { Agave americana } \\
\text { L. (BCN46860) }\end{array}$ & Figuerassa & Not reported & Leaf & Not reported & 1 \\
\hline & $\begin{array}{l}\text { Ruscus aculeatus } \\
\text { L. (BCN29939) }\end{array}$ & Galzeran. Galleranc & Cardiotonic & Root & Not reported & 1 \\
\hline \multirow[t]{2}{*}{ Asphodelaceae } & $\begin{array}{l}\text { Aloe maculata All. } \\
\text { (BCN50760) }\end{array}$ & - & Antipyrotic & Leaf & Direct use & 1 \\
\hline & $\begin{array}{l}\text { Aloe vera (L.) } \\
\text { Burm.f. } \\
\text { (BCN27242) }\end{array}$ & Àloe. Àloe vera & Antipyrotic. laxative. vulnerary & $\begin{array}{l}\text { Leaf. } \\
\text { inflorescence }\end{array}$ & Direct use. embrocation & 10 \\
\hline Aspleniaceae & $\begin{array}{l}\text { Ceterach } \\
\text { officinarum DC. in } \\
\text { Lam. et DC. } \\
\text { (BCN29850) }\end{array}$ & Dauradella & $\begin{array}{l}\text { Antihypertensive. blood } \\
\text { pressure regulator }\end{array}$ & Frond & Not reported. tisane & 2 \\
\hline \multirow[t]{3}{*}{ Asteraceae } & $\begin{array}{l}\text { Achillea ageratum } \\
\text { L. (BCN113701) }\end{array}$ & Herba del fàstic & Purgative & Inflorescence & Tisane & 1 \\
\hline & $\begin{array}{l}\text { Achillea } \\
\text { millefolium L. } \\
\text { (BCN113708) }\end{array}$ & $\begin{array}{l}\text { Cordonet. Herba de les } \\
\text { milfulles }\end{array}$ & Antineoplastic. emmenagogue & Inflorescence & Tisane & 2 \\
\hline & $\begin{array}{l}\text { Arnica montana L. } \\
\text { subsp. montana }\end{array}$ & Àrnica & $\begin{array}{l}\text { Anti-ecchymotic. antalgic/anti- } \\
\text { ecchymotic/anti-inflammatory. }\end{array}$ & Inflorescence & $\begin{array}{l}\text { Embrocation. lotion. } \\
\text { not reported }\end{array}$ & 13 \\
\hline
\end{tabular}


Table 2 Medicinal plants reported in the studied area (Continued)

\begin{tabular}{|c|c|c|c|c|c|c|}
\hline Family & Taxon (voucher) & Catalan vernacular names & Medicinal use & Part used & Pharmaceutical form & UR \\
\hline & (BCN29628) & & external antiseptic. for stings & & & \\
\hline & $\begin{array}{l}\text { Artemisia } \\
\text { absinthium L. } \\
\text { (BCN29837) }\end{array}$ & Artemisa. Donzell & $\begin{array}{l}\text { Abortive. antihelminthic. for } \\
\text { alcohol dishabituation }\end{array}$ & Aerial part & $\begin{array}{l}\text { Alcoholic tincture. not } \\
\text { reported }\end{array}$ & 3 \\
\hline & $\begin{array}{l}\text { Calendula arvensis } \\
\text { L. (BCN29637) }\end{array}$ & Lligamans & Ocular antiseptic & Aerial part & Bath & 1 \\
\hline & $\begin{array}{l}\text { Calendula } \\
\text { officinalis L. } \\
\text { (BCN29977) }\end{array}$ & Calèndula & $\begin{array}{l}\text { Anti-ecchymotic. } \\
\text { hepatoprotective }\end{array}$ & Inflorescence & Liniment. tisane & 2 \\
\hline & $\begin{array}{l}\text { Centaurea aspera } \\
\text { L. (BCN113579) }\end{array}$ & $\begin{array}{l}\text { Caps de burro. Flor del sucre. } \\
\text { Travalera }\end{array}$ & Hypoglycaemic & Aerial part & Not reported. tisane & 3 \\
\hline & $\begin{array}{l}\text { Inula helvetica } \\
\text { Weber } \\
\text { (BCN24668) }\end{array}$ & Àrnica borda & Anti-ecchymotic & Inflorescence & Lotion & 1 \\
\hline & $\begin{array}{l}\text { Matricaria recutita } \\
\text { L. (BCN113594) }\end{array}$ & Camamilla. Camamilla romana & $\begin{array}{l}\text { Analgesic. anticatarrhal. } \\
\text { antihelminthic. anti-nauseating. } \\
\text { digestive. external antiseptic. in- } \\
\text { ternal antiseptic. ocular antisep- } \\
\text { tic. stomachic }{ }^{\text {b. }}\end{array}$ & $\begin{array}{l}\text { Aerial part. } \\
\text { inflorescence. } \\
\text { not reported }\end{array}$ & Bath. emulsion. tisane & 46 \\
\hline & $\begin{array}{l}\text { Santolina } \\
\text { chamaecyparissus } \\
\text { L. (BCN113709) }\end{array}$ & Espernallac. Santolina & Digestive. not reported & $\begin{array}{l}\text { Inflorescence. } \\
\text { not reported }\end{array}$ & Tisane. not reported & 2 \\
\hline & $\begin{array}{l}\text { Sonchus oleraceus } \\
\text { L. (BCN113723) }\end{array}$ & Lletissó. Llipsó. Llistó & Diuretic & Aerial part & Not reported & 1 \\
\hline & $\begin{array}{l}\text { Tanacetum } \\
\text { vulgare L. } \\
\text { (BCN113712) }\end{array}$ & Camamilla de muntanya & Purgative $^{a}$ & Aerial part & Direct use & 1 \\
\hline & $\begin{array}{l}\text { Taraxacum } \\
\text { officinale Weber } \\
\text { in Wiggers } \\
\text { (BCN25948) }\end{array}$ & Dent de lleó. Xicoia & Hepatoprotective & Leaf & Direct use & 1 \\
\hline & $\begin{array}{l}\text { Tussilago farfara } \\
\text { L. (BCN29964) }\end{array}$ & Pota de cavall & $\begin{array}{l}\text { Antipyrotic. for undetermined } \\
\text { illnesses }\end{array}$ & Leaf & $\begin{array}{l}\text { Embrocation. not } \\
\text { reported }\end{array}$ & 2 \\
\hline Boraginaceae & $\begin{array}{l}\text { Lithospermum } \\
\text { officinale L. } \\
\text { (BCN113576) }\end{array}$ & Herba pedrera & Hepatic lithotriptic & Aerial part & Tisane & 1 \\
\hline & $\begin{array}{l}\text { Symphytum } \\
\text { tuberosum L. } \\
\text { (BCN22606) }\end{array}$ & Consolta & Vulnerary & Bulb & Ointment & 1 \\
\hline Brassicaceae & $\begin{array}{l}\text { Brassica napus L. } \\
\text { (BCN46856) }\end{array}$ & $\begin{array}{l}\text { Nap. Nap de bou. Nap del } \\
\text { camp }\end{array}$ & Restorative $^{a}$ & Root & Direct use & 1 \\
\hline & $\begin{array}{l}\text { Brassica oleracea } \\
\text { L. subsp. oleracea } \\
\text { (BCN32181) }\end{array}$ & Bròquil. Col. Col aloma & Analgesic & Leaf & Direct use & 2 \\
\hline Buxaceae & $\begin{array}{l}\text { Buxus } \\
\text { sempervirens L. } \\
\text { (BCN29843) }\end{array}$ & Boix & For skin disorders ${ }^{a}$ & Aerial part & Bath & 1 \\
\hline Cannabaceae & $\begin{array}{l}\text { Celtis australis L. } \\
\text { (BCN29845) }\end{array}$ & Lledó (fruit). Lledoner & $\begin{array}{l}\text { Anticholesterolemic. } \\
\text { antihypertensive. blood } \\
\text { pressure regulator. cardiotonic }\end{array}$ & Fruit. leaf & Not reported. tisane & 7 \\
\hline Caprifoliaceae & $\begin{array}{l}\text { Lonicera implexa } \\
\text { Ait. (BCN113802) }\end{array}$ & Lligabosc. Mareselva. Xuclamel & External antiseptic & Flower & Bath & 1 \\
\hline & $\begin{array}{l}\text { Scabiosa } \\
\text { atropurpurea L. } \\
\text { (BCN29947) }\end{array}$ & Escabiosa & $\begin{array}{l}\text { Anti-acne. antitussive. buccal } \\
\text { antiseptic. for scarlet fever. for } \\
\text { measles }\end{array}$ & $\begin{array}{l}\text { Aerial part. } \\
\text { flower. not } \\
\text { reported }\end{array}$ & Collutorium. tisane & 5 \\
\hline & $\begin{array}{l}\text { Valeriana } \\
\text { officinalis } \mathrm{L} \text {. }\end{array}$ & Valeriana & Abortive. sedative & Root & Tisane & 2 \\
\hline
\end{tabular}


Table 2 Medicinal plants reported in the studied area (Continued)

\begin{tabular}{|c|c|c|c|c|c|c|}
\hline Family & Taxon (voucher) & Catalan vernacular names & Medicinal use & Part used & Pharmaceutical form & UR \\
\hline & (BCN29816) & & & & & \\
\hline Caryophyllaceae & $\begin{array}{l}\text { Herniaria glabra L. } \\
\text { (BCN113577) }\end{array}$ & $\begin{array}{l}\text { Herba de les mil granes. Mil } \\
\text { granes }\end{array}$ & Diuretic, renal anti-inflammatory & Aerial part & Tisane & 4 \\
\hline Cistaceae & $\begin{array}{l}\text { Cistus } \\
\text { monspeliensis L. } \\
\text { (BCN36740) }\end{array}$ & Estepa. Mòdega & Antidiarrhoeal & Leaf & Tisane & 1 \\
\hline Clusiaceae & $\begin{array}{l}\text { Hypericum } \\
\text { perforatum L. } \\
\text { (BCN113597) }\end{array}$ & $\begin{array}{l}\text { Flor de Sant Joan. Herba de } \\
\text { cop. Herba de Sant Joan }\end{array}$ & $\begin{array}{l}\text { Anti-ecchymotic. antipyrotic. } \\
\text { gastric anti-inflammatory. renal } \\
\text { anti-inflammatory. vulnerary }\end{array}$ & $\begin{array}{l}\text { Aerial part. } \\
\text { flower. not } \\
\text { reported }\end{array}$ & $\begin{array}{l}\text { Embrocation. liniment. } \\
\text { lotion. not reported }\end{array}$ & 32 \\
\hline Cneoraceae & $\begin{array}{l}\text { Cneorum } \\
\text { tricoccon L. } \\
\text { (BCN51285) }\end{array}$ & Olivereta & Antihypertensive & Leaf & Tisane & 1 \\
\hline \multirow[t]{2}{*}{ Crassulaceae } & $\begin{array}{l}\text { Sedum sediforme } \\
\text { (Jacq.) Pau } \\
\text { (BCN29792) }\end{array}$ & - & Cicatrizing & Leaf & Direct use & 1 \\
\hline & $\begin{array}{l}\text { Sedum telephium } \\
\text { L. (BCN24995) }\end{array}$ & Bàlsam & Antipyrotic & Leaf & Direct use & 1 \\
\hline \multirow[t]{2}{*}{ Cucurbitaceae } & $\begin{array}{l}\text { Cucumis sativus L. } \\
\text { (BCN46850) }\end{array}$ & Cogombre (fruit) & $\begin{array}{l}\text { Antihaemorrhoidal. antivaricose. } \\
\text { gastric anti-inflammatory }\end{array}$ & Fruit & Liniment & 3 \\
\hline & $\begin{array}{l}\text { Cucurbita pepo L. } \\
\text { var. pepo } \\
\text { (BCN49858) }\end{array}$ & $\begin{array}{l}\text { Carbassa (fruit). Carbassera. } \\
\text { Rabequet (fruit) }\end{array}$ & $\begin{array}{l}\text { Antihelminthic. for abscesses. } \\
\text { for skin disorders. prostate anti- } \\
\text { inflammatory. renal lithotriptic }\end{array}$ & Fruit. seed & Not reported. ointment & 6 \\
\hline \multirow[t]{2}{*}{ Cupressaceae } & $\begin{array}{l}\text { Juniperus } \\
\text { communis L. } \\
\text { (BCN113589) }\end{array}$ & Ginebre. Ginebró & Analgesic. for scabies ${ }^{a}$ & $\begin{array}{l}\text { Fructification. } \\
\text { root }\end{array}$ & $\begin{array}{l}\text { Liniment. lotion. } \\
\text { poultice }\end{array}$ & 3 \\
\hline & $\begin{array}{l}\text { Juniperus } \\
\text { oxycedrus L. } \\
\text { (BCN29879) }\end{array}$ & Càdec & Not reported & Aerial part & Not reported & 1 \\
\hline \multirow[t]{3}{*}{ Equisetaceae } & $\begin{array}{l}\text { Equisetum arvense } \\
\text { L. (BCN24767) }\end{array}$ & Cua de cavall. Sangnua & Diuretic. not reported & Aerial part & Direct use. not reported & 2 \\
\hline & Equisetum sp. & Cua de cavall. Sangnua & $\begin{array}{l}\text { Analgesic. antihypertensive. } \\
\text { buccal antiseptic. diuretic. } \\
\text { urinary antiseptic }\end{array}$ & Arial part & Collutorium. tisane & 12 \\
\hline & $\begin{array}{l}\text { Equisetum } \\
\text { telmateia Ehrh. } \\
\text { (BCN113581) }\end{array}$ & Cua de cavall. Sangnua & $\begin{array}{l}\text { Diuretic. for iron-deficiency. } \\
\text { renal lithotriptic. salutiferous }\end{array}$ & Aerial part & Tisane & 8 \\
\hline \multirow[t]{3}{*}{ Euphorbiaceae } & Euphorbia sp. & Lletdetereses. Lletdetresa & For warts & Latex & Direct use & 4 \\
\hline & $\begin{array}{l}\text { Mercurialis annua } \\
\text { L. (BCN29896) }\end{array}$ & Blet. Murcarol & Laxative & Aerial part & Tisane & 1 \\
\hline & $\begin{array}{l}\text { Ricinus communis } \\
\text { L. (BCN46089) }\end{array}$ & Oli de ricí (elaborated product) & Purgative & Fruit & Direct use & 1 \\
\hline \multirow[t]{3}{*}{ Fabaceae } & $\begin{array}{l}\text { Ceratonia siliqua } \\
\text { L. (BCN32177) }\end{array}$ & Garrofa (fruit) & Salutiferous $^{a}$ & Fruit & Direct use & 1 \\
\hline & $\begin{array}{l}\text { Medicago sativa L. } \\
\text { (BCN29891) }\end{array}$ & Userda & $\begin{array}{l}\text { Analgesic. anti-ecchymotic. not } \\
\text { reported }\end{array}$ & Aerial part & Poultice & 4 \\
\hline & $\begin{array}{l}\text { Spartium junceum } \\
\text { L. (BCN29956) }\end{array}$ & Ginesta & $\begin{array}{l}\text { Anti-ecchymotic. insects } \\
\text { repellent }^{\mathrm{a}}\end{array}$ & Flower & Liniment & 2 \\
\hline Fagaceae & $\begin{array}{l}\text { Quercus ilex L. } \\
\text { (BCN113730) }\end{array}$ & $\begin{array}{l}\text { Aglà (fruit). Alzina. Aulina. Gla } \\
\text { (fruit) }\end{array}$ & $\begin{array}{l}\text { Antibronchitic. antidiarrhoeal }{ }^{\mathrm{a}} \text {. } \\
\text { cicatrizing }^{\mathrm{b}} \text {. for amygdalitis }\end{array}$ & $\begin{array}{l}\text { Bark. in situ } \\
\text { living plant. } \\
\text { leaf. stem }\end{array}$ & $\begin{array}{l}\text { Bath. colloidal solution. } \\
\text { direct use }\end{array}$ & 5 \\
\hline Gesneriaceae & $\begin{array}{l}\text { Ramonda myconi } \\
\text { (L.) Reichenb. } \\
\text { (BCN46088) }\end{array}$ & Orella d'os & $\begin{array}{l}\text { Anticatarrhal. } \\
\text { antihaemorrhoidal. antiherpes. } \\
\text { antipneumonic. } \\
\text { antipyretic, antitussive. } \\
\text { pharyngeal anti-inflammatory. } \\
\text { postpartum coadjuvant }{ }^{\mathrm{a}} \text {. } \\
\text { stomachic }\end{array}$ & $\begin{array}{l}\text { Aerial part. } \\
\text { leaf }\end{array}$ & $\begin{array}{l}\text { Embrocation. not } \\
\text { reported. tisane }\end{array}$ & 15 \\
\hline
\end{tabular}


Table 2 Medicinal plants reported in the studied area (Continued)

\begin{tabular}{|c|c|c|c|c|c|c|}
\hline Family & Taxon (voucher) & Catalan vernacular names & Medicinal use & Part used & Pharmaceutical form & $\overline{U R}$ \\
\hline Juglandaceae & $\begin{array}{l}\text { Juglans regia L. } \\
\text { (BCN29877) }\end{array}$ & $\begin{array}{l}\text { Noguer. Nou (fruit). Nou verda } \\
\text { (fruit) }\end{array}$ & Antialopecia. antihypertensive & Leaf & Bath. tisane & 2 \\
\hline \multirow[t]{21}{*}{ Lamiaceae } & $\begin{array}{l}\text { Hyssopus } \\
\text { officinalis L. } \\
\text { (BCN29709) }\end{array}$ & Hisop & Anticatarrhal & Aerial part & Tisane & 1 \\
\hline & $\begin{array}{l}\text { Lavandula } \\
\text { dentata L. } \\
\text { (BCN29715) }\end{array}$ & Lavanda & Anti-inflammatory & Flower & Direct use & 1 \\
\hline & $\begin{array}{l}\text { Lavandula } \\
\text { stoechas L. } \\
\text { (BCN113714) }\end{array}$ & Cap d'ase. Tomanyí & Stomachic & Flower & Tisane & 2 \\
\hline & $\begin{array}{l}\text { Melissa officinalis } \\
\text { L. (BCN113713) }\end{array}$ & Melissa. Tarongina & Tranquilizer & Aerial part & Tisane & 1 \\
\hline & $\begin{array}{l}\text { Mentha xpiperita } \\
\text { L. (BCN113813) }\end{array}$ & $\begin{array}{l}\text { Menta. Menta de la xocolata. } \\
\text { Menta piperita. Menta romana }\end{array}$ & Stomachic & Aerial part & Tisane & 1 \\
\hline & $\begin{array}{l}\text { Mentha pulegium } \\
\text { L. (BCN113598) }\end{array}$ & Poliol. Poniol & $\begin{array}{l}\text { Antidiarrhoeal. antihypertensive. } \\
\text { digestive. intestinal anti- } \\
\text { inflammatory. tranquilizer }\end{array}$ & $\begin{array}{l}\text { Aerial part. } \\
\text { flower }\end{array}$ & Tisane & 24 \\
\hline & $\begin{array}{l}\text { Mentha spicata L. } \\
\text { (BCN113812) }\end{array}$ & $\begin{array}{l}\text { Menta. Menta de la sopa. } \\
\text { Menta silvestre. Menta espicata. } \\
\text { Menta verdadera }\end{array}$ & $\begin{array}{l}\text { Emmenagogue. for stings. } \\
\text { intestinal anti-inflammatory }\end{array}$ & $\begin{array}{l}\text { Aerial part. } \\
\text { leaf }\end{array}$ & $\begin{array}{l}\text { Direct use. emulsion. } \\
\text { poultice. tisane }\end{array}$ & 8 \\
\hline & $\begin{array}{l}\text { Origanum } \\
\text { majorana L. } \\
\text { (BCN113585) }\end{array}$ & Marduix & For earache & Aerial part & $\begin{array}{l}\text { Embrocation. not } \\
\text { reported }\end{array}$ & 2 \\
\hline & $\begin{array}{l}\text { Origanum vulgare } \\
\text { L. (BCN113705) }\end{array}$ & Orenga & Restorative. stomachic & Aerial part & Tisane & 3 \\
\hline & $\begin{array}{l}\text { Prunella vulgaris } \mathrm{L} . \\
\text { (BCN113578) }\end{array}$ & Herba del traïdor & Anti-acne & Aerial part & Tisane & 1 \\
\hline & $\begin{array}{l}\text { Rosmarinus } \\
\text { officinalis L. } \\
\text { (BCN113599) }\end{array}$ & Romaní & $\begin{array}{l}\text { Analgesic. anticatarrhal. } \\
\text { antidepressant. anti-ecchymotic }\end{array}$ & Aerial part & $\begin{array}{l}\text { Liniment. lotion. } \\
\text { medicinal wine. tisane }\end{array}$ & 14 \\
\hline & $\begin{array}{l}\text { Salvia officinalis L. } \\
\text { subsp. officinalis } \\
\text { (BCN113583) }\end{array}$ & Sàlvia. Sàlvia de fulla ampla & $\begin{array}{l}\text { Analgesic. antihypertensive. for } \\
\text { fatigue. hematocathartic. not } \\
\text { reported. sedative }\end{array}$ & $\begin{array}{l}\text { Aerial part. } \\
\text { not reported }\end{array}$ & $\begin{array}{l}\text { Collutorium. not } \\
\text { reported. tisane }\end{array}$ & 10 \\
\hline & $\begin{array}{l}\text { Salvia verbenaca } \\
\text { L. (BCN113580) }\end{array}$ & Herba de les iaies & Antipyertensive & Flower & Tisane & 1 \\
\hline & $\begin{array}{l}\text { Satureja } \\
\text { calamintha (L.) } \\
\text { Scheele } \\
\text { (BCN113737) }\end{array}$ & Menta blava & Digestive & Aerial part & Tisane & 1 \\
\hline & $\begin{array}{l}\text { Satureja montana } \\
\text { L. (BCN113741) }\end{array}$ & Sajolida & Hematocathartic & Aerial part & Tisane & 1 \\
\hline & $\begin{array}{l}\text { Sideritis hirsuta L. } \\
\text { (BCN113582) }\end{array}$ & Herba de Sant Antoni & Vasotonic & Aerial part & Tisane & 1 \\
\hline & $\begin{array}{l}\text { Stachys byzantina } \\
\text { C. Koch } \\
\text { (BCN113707) }\end{array}$ & $\begin{array}{l}\text { Fulles de la mare de Déu. } \\
\text { Planta de vellut }\end{array}$ & $\begin{array}{l}\text { Antipyrotic. cicatrizing. } \\
\text { vulnerary }\end{array}$ & Leaf & Direct use. embrocation & 8 \\
\hline & $\begin{array}{l}\text { Stachys officinalis } \\
\text { (L.) Trevisan } \\
\text { (BCN25011) }\end{array}$ & Brotònica & Antihypertensive & Aerial part & Tisane & 2 \\
\hline & $\begin{array}{l}\text { Teucrium } \\
\text { chamaedrys L. } \\
\text { (BCN29806) }\end{array}$ & Brotònica & Anticatarrhal & Aerial part & Not reported & 1 \\
\hline & $\begin{array}{l}\text { Thymus serpyllum } \\
\text { L. (BCN113719) }\end{array}$ & $\begin{array}{l}\text { Farigola de pastor. Farigoleta. } \\
\text { Salsa de pastor }\end{array}$ & $\begin{array}{l}\text { Internal antiseptic. not reported. } \\
\text { stomachic }\end{array}$ & Aerial part & Tisane & 3 \\
\hline & $\begin{array}{l}\text { Thymus vulgaris } \mathrm{L} \text {. } \\
\text { (BCN113590) }\end{array}$ & Farigola & $\begin{array}{l}\text { Anticatarrhal. anti- } \\
\text { inflammatory }{ }^{\mathrm{a}} \text {. buccal antiseptic. }\end{array}$ & Aerial part & $\begin{array}{l}\text { Bath. collutorium. direct } \\
\text { use, emulsion. }\end{array}$ & 78 \\
\hline
\end{tabular}


Table 2 Medicinal plants reported in the studied area (Continued)

\begin{tabular}{|c|c|c|c|c|c|c|}
\hline Family & Taxon (voucher) & Catalan vernacular names & Medicinal use & Part used & Pharmaceutical form & UR \\
\hline & & & 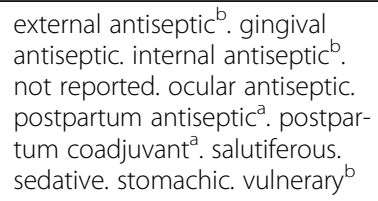 & & $\begin{array}{l}\text { fumigation. gargle. } \\
\text { liniment. tisane }\end{array}$ & \\
\hline \multirow[t]{2}{*}{ Lauraceae } & $\begin{array}{l}\text { Cinnamomum } \\
\text { zeylanicum Nees } \\
\text { (BCN47283) }\end{array}$ & Canyella & Anticholesterolemic & Bark & Direct use & 1 \\
\hline & $\begin{array}{l}\text { Laurus nobilis L. } \\
\text { (BCN113717) }\end{array}$ & Llorer. Llort & $\begin{array}{l}\text { Analgesic. anticatarrhal. } \\
\text { expectorant. not reported }\end{array}$ & Leaf & $\begin{array}{l}\text { Aerosol. bath. not } \\
\text { reported }\end{array}$ & 6 \\
\hline Liliaceae & $\begin{array}{l}\text { Lilium candidum } \\
\text { L. (BCN46841) }\end{array}$ & $\begin{array}{l}\text { Lliri de Sant Antoni. Lliri de } \\
\text { Sant Josep }\end{array}$ & $\begin{array}{l}\text { Antipyrotic. external antiseptic. } \\
\text { vulnerary }\end{array}$ & Flower. leaf & $\begin{array}{l}\text { Embrocation. not } \\
\text { reported }\end{array}$ & 8 \\
\hline Linaceae & $\begin{array}{l}\text { Linum } \\
\text { usitatissimum L. } \\
\text { (BCN47281) }\end{array}$ & $\begin{array}{l}\text { Farina de llinet (elaborated } \\
\text { product). Llinet }\end{array}$ & 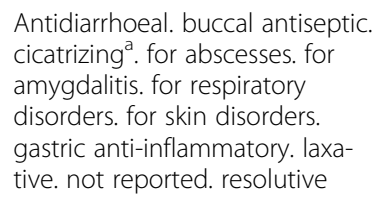 & Seed & Decoction. poultice & 15 \\
\hline Lythraceae & $\begin{array}{l}\text { Punica granatum } \\
\text { L. (BCN29764) }\end{array}$ & $\begin{array}{l}\text { Magrana (fruit). Magraner. } \\
\text { Magraner agre. Magraner bord. } \\
\text { Magraner dolç }\end{array}$ & Antihelminthic & Fruit. root & Decoction. direct use & 3 \\
\hline \multirow[t]{5}{*}{ Malvaceae } & $\begin{array}{l}\text { Althaea officinalis } \\
\text { L. (BCN113799) }\end{array}$ & Malví & Not reported & Root & Not reported & 1 \\
\hline & $\begin{array}{l}\text { Malva sylvestris L. } \\
\text { (BCN29889) }\end{array}$ & Malva. Malva rosa & $\begin{array}{l}\text { Anticatarrhal. antipyrotic. not } \\
\text { reported }\end{array}$ & $\begin{array}{l}\text { Aerial part. } \\
\text { flower. leaf }\end{array}$ & $\begin{array}{l}\text { Not reported. poultice. } \\
\text { tisane }\end{array}$ & 3 \\
\hline & $\begin{array}{l}\text { Theobroma cacao } \\
\text { L. (BCN30763) }\end{array}$ & Xocolata (elaborated product) & Antihelminthic & Seed & Direct use & 1 \\
\hline & $\begin{array}{l}\text { Tilia cordata Mill. } \\
\text { (BCN26784) }\end{array}$ & Tilla & $\begin{array}{l}\text { For headache. not reported. } \\
\text { tranquilizer }\end{array}$ & $\begin{array}{l}\text { Bract with } \\
\text { inflorescence }\end{array}$ & Tisane & 5 \\
\hline & $\begin{array}{l}\text { Tilia platyphyllos } \\
\text { Scop. } \\
\text { (BCN113739) }\end{array}$ & Tei. Til.la. Til.ler de bosc & $\begin{array}{l}\text { Anticatarrhal. antihypertensive. } \\
\text { tranquilizer }\end{array}$ & $\begin{array}{l}\text { Bract with } \\
\text { inflorescence }\end{array}$ & Tisane & 8 \\
\hline Moraceae & $\begin{array}{l}\text { Ficus carica L. } \\
\text { (BCN24887) }\end{array}$ & $\begin{array}{l}\text { Figa (infructescence). Figa } \\
\text { d'Alacant (infructescence). Figa } \\
\text { de coll de senyora } \\
\text { (infructescence). Figa de coll } \\
\text { llarg blanca (infructescence). } \\
\text { Figa de coll llarg negra } \\
\text { (infructescence). Figa de pota } \\
\text { de cavall (infructescence). Figa } \\
\text { de Sant Joan (infructescence). } \\
\text { Figa negra (infructescence). } \\
\text { Figuera. Figuera de coll de } \\
\text { senyora }\end{array}$ & For warts & Latex & Direct use & 5 \\
\hline Myrtaceae & $\begin{array}{l}\text { Eucalyptus } \\
\text { globulus Labill. } \\
\text { (BCN29696) }\end{array}$ & Eucaliptu. Eucaliptus & $\begin{array}{l}\text { Anticatarrhal. expectorant. for } \\
\text { respiratory disorders }\end{array}$ & Leaf & Aerosol. tisane & 18 \\
\hline Oleaceae & $\begin{array}{l}\text { Olea europaea L. } \\
\text { subsp. europaea } \\
\text { (BCN29898) }\end{array}$ & $\begin{array}{l}\text { Oli (elaborated product). Oli } \\
\text { d'oliva (elaborated product). } \\
\text { Olivera. Oliva (fruit) }\end{array}$ & $\begin{array}{l}\text { Antihelminthic. } \\
\text { antihypertensive. } \\
\text { antihypotensive. antipyrotic. } \\
\text { blood pressure regulator. } \\
\text { cicatrizing. external antiseptic. } \\
\text { for earache. for mastitis. for skin } \\
\text { disorders. vulnerary }\end{array}$ & $\begin{array}{l}\text { Flower. fruit. } \\
\text { leaf }\end{array}$ & $\begin{array}{l}\text { Direct use. } \\
\text { embrocation. emulsion. } \\
\text { fumigation. not } \\
\text { reported. ointment. } \\
\text { tisane }\end{array}$ & 41 \\
\hline Paeoniaceae & $\begin{array}{l}\text { Paeonia officinalis } \\
\text { L. (BCN29320) }\end{array}$ & Peònia & Not reported & Root & Not reported & 1 \\
\hline Papaveraceae & $\begin{array}{l}\text { Chelidonium } \\
\text { majus L. } \\
\text { (BCN113742) }\end{array}$ & $\begin{array}{l}\text { Berruguera. Celoni. Herba de } \\
\text { les orenetes. Llet de Santa } \\
\text { Teresa }\end{array}$ & For warts & Latex & Direct use & 4 \\
\hline
\end{tabular}


Table 2 Medicinal plants reported in the studied area (Continued)

\begin{tabular}{|c|c|c|c|c|c|c|}
\hline Family & Taxon (voucher) & Catalan vernacular names & Medicinal use & Part used & Pharmaceutical form & UR \\
\hline & $\begin{array}{l}\text { Papaver rhoeas L. } \\
\text { (BCN29903) }\end{array}$ & $\begin{array}{l}\text { Gallaret. Pipiripip. Quiquiriquí. } \\
\text { Rosella }\end{array}$ & Analgesic. sedative & Seed & Direct use. not reported & 2 \\
\hline & $\begin{array}{l}\text { Papaver } \\
\text { somniferum L. } \\
\text { (BCN24941) }\end{array}$ & Cascall & Analgesic. sedative & $\begin{array}{l}\text { Flower. fruit. } \\
\text { seed }\end{array}$ & $\begin{array}{l}\text { Collutorium. direct use. } \\
\text { not reported. tisane }\end{array}$ & 10 \\
\hline \multirow[t]{4}{*}{ Pinaceae } & $\begin{array}{l}\text { Pinus halepensis } \\
\text { Mill. (BCN113592) }\end{array}$ & $\begin{array}{l}\text { Pi. Pi blanc. Pi bord. Pi de pinya } \\
\text { llarga. Pi petit. Pinya } \\
\text { (fructification) }\end{array}$ & $\begin{array}{l}\text { Antibronchitic. anticatarrhal. } \\
\text { antipneumonic. antitussive. } \\
\text { expectorant. for abscesses. not } \\
\text { reported. vulnerary }{ }^{\mathrm{a}}\end{array}$ & $\begin{array}{l}\text { Aerial part. } \\
\text { flower. fruit. } \\
\text { gum/resin. } \\
\text { leaf. pollen }\end{array}$ & $\begin{array}{l}\text { Decoction. fumigation. } \\
\text { liniment. lotion. not } \\
\text { reported. syrup. tisane }\end{array}$ & 33 \\
\hline & $\begin{array}{l}\text { Pinus pinaster Ait. } \\
\text { (BCN36559) }\end{array}$ & Pi bord. Pi melis & Antibronchitic. antirheumatic & Fruit & Decoction. syrup & 2 \\
\hline & $\begin{array}{l}\text { Pinus pinea L. } \\
\text { (BCN26751) }\end{array}$ & $\begin{array}{l}\text { Pi. Pi de llei. Pi de pinya. Pi } \\
\text { pinyer }\end{array}$ & Antibronchitic & Fruit. leaf & Aerosol. syrup & 2 \\
\hline & Pinus sp. & $\begin{array}{l}\text { Pi. Trementina (elaborated } \\
\text { product) }\end{array}$ & Anti-ecchymotic & Gum/resin & Not reported & 1 \\
\hline \multirow[t]{2}{*}{ Plantaginaceae } & $\begin{array}{l}\text { Plantago } \\
\text { lanceolata L. } \\
\text { (BCN32138) }\end{array}$ & $\begin{array}{l}\text { Plantatge de fulla estreta. } \\
\text { Plantatge estret }\end{array}$ & Gingival antiseptic & Leaf & Collutorium & 2 \\
\hline & $\begin{array}{l}\text { Plantago major L. } \\
\text { (BCN29910) }\end{array}$ & $\begin{array}{l}\text { Plantatge. Plantatge ample. } \\
\text { Plantatge de fulla ampla }\end{array}$ & $\begin{array}{l}\text { Buccal antiseptic. external } \\
\text { antiseptic. for amygdalitis. } \\
\text { gingival antiseptic. not } \\
\text { reported. vulvar anti- } \\
\text { inflammatory }\end{array}$ & $\begin{array}{l}\text { Aerial part. } \\
\text { leaf }\end{array}$ & $\begin{array}{l}\text { Bath. collutorium. } \\
\text { gargle. not reported }\end{array}$ & 8 \\
\hline \multirow[t]{4}{*}{ Poaceae } & $\begin{array}{l}\text { Arundo donax L. } \\
\text { (BCN29825) }\end{array}$ & $\begin{array}{l}\text { Canya. Canya americana. } \\
\text { Canyer }\end{array}$ & For trauma & Stem & Direct use & 1 \\
\hline & $\begin{array}{l}\text { Phleum phleoides } \\
\text { (L.) Karsten } \\
\text { (BCN113804) }\end{array}$ & Herba de les pedres & Analgesic & Inflorescence & Tisane & 1 \\
\hline & $\begin{array}{l}\text { Triticum aestivum } \\
\text { L. (BCN29963) }\end{array}$ & $\begin{array}{l}\text { Blat. Farina (elaborated } \\
\text { product). Pa (elaborated } \\
\text { product). Palla (elaborated } \\
\text { product). Segó (bran) }\end{array}$ & $\begin{array}{l}\text { Antidiarrhoeal }{ }^{a} \text {. antihelminthic. } \\
\text { internal antiseptic }{ }^{a} \text {. postpartum } \\
\text { coadjuvant }^{\mathrm{a}} \text {. restorative } \mathrm{e}^{\mathrm{a}}\end{array}$ & Bran. fruit & $\begin{array}{l}\text { Direct use. emulsion. } \\
\text { poultice. solution }\end{array}$ & 14 \\
\hline & $\begin{array}{l}\text { Zea mays L. } \\
\text { (BCN29830) }\end{array}$ & $\begin{array}{l}\text { Blat de morassa. Blat de moret. } \\
\text { Blat de moro. Farro (elaborated } \\
\text { product) }\end{array}$ & $\begin{array}{l}\text { Diuretic. renal anti- } \\
\text { inflammatory.. renal lithotriptic. } \\
\text { urinary antiseptic }\end{array}$ & $\begin{array}{l}\text { Styles and } \\
\text { stigmas }\end{array}$ & Tisane & 20 \\
\hline \multirow[t]{2}{*}{ Ranunculaceae } & $\begin{array}{l}\text { Anemone } \\
\text { hepatica L. } \\
\text { (BCN29834) }\end{array}$ & Herba fetgera & $\begin{array}{l}\text { For undetermined } \\
\text { illnesses }^{a} \text {, hepatoprotective }\end{array}$ & Flower. leaf & $\begin{array}{l}\text { Direct use. not } \\
\text { reported. tisane }\end{array}$ & 11 \\
\hline & $\begin{array}{l}\text { Clematis } \\
\text { flammula L. } \\
\text { (BCN29856) }\end{array}$ & Viadella. Virobella & For warts. not reported & Leaf & Direct use. poultice & 3 \\
\hline \multirow[t]{6}{*}{ Rosaceae } & $\begin{array}{l}\text { Agrimonia } \\
\text { eupatoria L. (BCN- } \\
\text { E-193) }\end{array}$ & Herba cuquera & Antihelminthic & Flower & Tisane & 2 \\
\hline & $\begin{array}{l}\text { Crataegus } \\
\text { monogyna Jacq. } \\
\text { (BCN29858) }\end{array}$ & Arç. Arç blanc & Antihypertensive. cardiotonic & Flower & Tisane & 3 \\
\hline & $\begin{array}{l}\text { Cydonia oblonga } \\
\text { Mill. (BCN46849) }\end{array}$ & $\begin{array}{l}\text { Codony (fruit). Codonyat } \\
\text { (elaborated product). Codonyer }\end{array}$ & $\begin{array}{l}\text { Antidiarrhoeal. antitussive. not } \\
\text { reported. stomachic }\end{array}$ & Fruit & $\begin{array}{l}\text { Alcoholic } \\
\text { tincture, decoction, not } \\
\text { reported. syrup }\end{array}$ & 15 \\
\hline & $\begin{array}{l}\text { Potentilla reptans } \\
\text { L. (BCN29754) }\end{array}$ & Gram negre & Antihypertensive & Root & Tisane & 1 \\
\hline & $\begin{array}{l}\text { Prunus avium (L.) } \\
\text { L. (BCN29827) }\end{array}$ & Cirera (fruit). Cirerer & Diuretic, for the influenza & Stem & Tisane & 2 \\
\hline & Pyrus malus L. & Poma (fruit). Poma aspra (fruit). & Anticatarrhal. restorative & Fruit & Direct use & 3 \\
\hline
\end{tabular}

subsp. mitis Poma cambusina (fruit). Poma

(Wallr.) O.Bolòs et camosa (fruit). Poma capçana

J.Vigo (BCN46830) (fruit). Poma del ciri (fruit). 
Table 2 Medicinal plants reported in the studied area (Continued)

\begin{tabular}{|c|c|c|c|c|c|c|}
\hline Family & Taxon (voucher) & Catalan vernacular names & Medicinal use & Part used & Pharmaceutical form & UR \\
\hline & & $\begin{array}{l}\text { Poma del ciri groga (fruit). } \\
\text { Poma del ciri vermella (fruit). } \\
\text { Poma golden (fruit). Poma } \\
\text { rodona (fruit). Poma royal } \\
\text { (fruit). Pomer. Pomer del ciri. } \\
\text { Pomera. Pomera del ciri }\end{array}$ & & & & \\
\hline & $\begin{array}{l}\text { Rosa canina L. } \\
\text { (BCN29772) }\end{array}$ & Rosa. Rosa de pastor. Roser & Anticatarrhal & Fruit & Tisane & 1 \\
\hline & $\begin{array}{l}\text { Rubus ulmifolius } \\
\text { Schott } \\
\text { (BCN29938) }\end{array}$ & $\begin{array}{l}\text { Bardissa. Mora (fruit). Mora } \\
\text { negra (fruit). Romeguera }\end{array}$ & $\begin{array}{l}\text { Antidiarrhoeal. for stings. } \\
\text { pharyngeal anti-inflammatory. } \\
\text { vulnerary }\end{array}$ & $\begin{array}{l}\text { Leaf. young } \\
\text { shoot }\end{array}$ & Direct use. gargle & 4 \\
\hline & $\begin{array}{l}\text { Sanguisorba } \\
\text { minor Scop. } \\
\text { (BCN113728) }\end{array}$ & Esparcet bord & Antdiarrhoeal & Aerial part & Tisane & 1 \\
\hline \multirow[t]{2}{*}{ Rubiaceae } & $\begin{array}{l}\text { Asperula } \\
\text { cynanchica L. } \\
\text { (BCN29634) }\end{array}$ & Herba prima & $\begin{array}{l}\text { Diuretic. intestinal anti- } \\
\text { inflammatory. renal lithotriptic. } \\
\text { urinary antiseptic }\end{array}$ & Aerial part & Tisane & 16 \\
\hline & $\begin{array}{l}\text { Coffea arabica L. } \\
(\mathrm{BCN} 46852)^{\mathrm{C}}\end{array}$ & Cafè & Antihypotensive & Seed & Tisane & 2 \\
\hline \multirow[t]{3}{*}{ Rutaceae } & $\begin{array}{l}\text { Citrus limon (L.) } \\
\text { Burm. (BCN46853) }\end{array}$ & Llimona (fruit). Llimoner & $\begin{array}{l}\text { Anticatarrhal. anti-eccymotic. } \\
\text { antihypertensive. digestive. for } \\
\text { amygdalitis. restorative }\end{array}$ & Fruit & $\begin{array}{l}\text { Direct use. gargle. not } \\
\text { reported. tisane }\end{array}$ & 9 \\
\hline & $\begin{array}{l}\text { Citrus sinensis (L.) } \\
\text { Osbeck } \\
\text { (BCN24752) }\end{array}$ & $\begin{array}{l}\text { Taronger. Taronger dolç. } \\
\text { Taronja (fruit) }\end{array}$ & Anticatarrhal & Fruit & Direct use & 1 \\
\hline & $\begin{array}{l}\text { Ruta chalepensis } \\
\text { L. (BCN29940) }\end{array}$ & Ruda & $\begin{array}{l}\text { Abortive }^{\mathrm{b}} \text {. analgesic } \\
\text { antihelminthic }^{\mathrm{b}} \text {. } \text { anti- }^{-} \\
\text {inflammatory/antiseptic/cicatriz- } \\
\text { ing mucronal. } \\
\text { amygdiuretic, for } \\
\text { orders. laxative }^{\mathrm{b}} \text {. not reported. } \\
\text { ocular antiseptic. } \\
\text { ruminant antistatic }\end{array}$ & Aerial part & $\begin{array}{l}\text { Bath. direct use. } \\
\text { liniment. poultice. not } \\
\text { reported. tisane }\end{array}$ & 38 \\
\hline Smilacaceae & $\begin{array}{l}\text { Smilax aspera L. } \\
\text { (BCN29951) }\end{array}$ & Arítjol & Analgesic. antihypertensive & Root & Decoction. liniment & 2 \\
\hline \multirow[t]{3}{*}{ Solanaceae } & $\begin{array}{l}\text { Nicotiana } \\
\text { tabacum L. } \\
\text { (BCN48711) }\end{array}$ & Tabac & Antihelminthic. antitussive & Leaf & Direct use. fumigation & 3 \\
\hline & $\begin{array}{l}\text { Solanum } \\
\text { melongena L. } \\
\text { (BCN25004) }\end{array}$ & Albergínia & For warts & Fruit & Direct use & 1 \\
\hline & $\begin{array}{l}\text { Solanum } \\
\text { tuberosum L. } \\
\text { (BCN29797) }\end{array}$ & Patata. Patatera. Trumfera & Antipyrotic & Tuber & Direct use & 1 \\
\hline Thymelaeaceae & $\begin{array}{l}\text { Daphne gnidium } \\
\text { L. (BCN29687) }\end{array}$ & - & Antidiarrhoeal ${ }^{a}$ & Aerial part & Direct use & 1 \\
\hline Ulmaceae & $\begin{array}{l}\text { Ulmus minor Mill. } \\
\text { (BCN113729) }\end{array}$ & Om & Anticholesterolemic. antipyrotic & Bark. leaf & Bath. tisane & 3 \\
\hline \multirow[t]{2}{*}{ Urticaceae } & $\begin{array}{l}\text { Parietaria } \\
\text { officinalis L. } \\
\text { subsp. judaica (L.) } \\
\text { Béguinot } \\
\text { (BCN113715) }\end{array}$ & Blet de paret. Mollerosa & $\begin{array}{l}\text { Analgesic. anticatarrhal. } \\
\text { antihaemorrhoidal. buccal } \\
\text { antiseptic. digestive. for } \\
\text { digestive disorders. for stings. } \\
\text { for urticaria. postpartum } \\
\text { coadjuvant. urinary antiseptic. } \\
\text { vaginal antiseptic }\end{array}$ & Aerial part & $\begin{array}{l}\text { Bath. collutorium. direct } \\
\text { use. not reported. } \\
\text { poultice. tisane }\end{array}$ & 15 \\
\hline & $\begin{array}{l}\text { Urtica dioica L. } \\
\text { (BCN29814) }\end{array}$ & Ortiga & $\begin{array}{l}\text { Against prurigo. } \\
\text { antieritematous. } \\
\text { antihypertensive. }\end{array}$ & $\begin{array}{l}\text { Aerial part. } \\
\text { root }\end{array}$ & $\begin{array}{l}\text { Bath. decoction. direct } \\
\text { use. not reported. } \\
\text { tisane }\end{array}$ & 10 \\
\hline
\end{tabular}


Table 2 Medicinal plants reported in the studied area (Continued)

\begin{tabular}{|c|c|c|c|c|c|c|}
\hline Family & Taxon (voucher) & Catalan vernacular names & Medicinal use & Part used & Pharmaceutical form & UR \\
\hline & & & $\begin{array}{l}\text { hemathocathartic. not reported. } \\
\text { vasotonic }\end{array}$ & & & \\
\hline & $\begin{array}{l}\text { Urtica urens L. } \\
\text { (BCN29966) }\end{array}$ & Ortiga de fulla petita & Anticatarrhal & Aerial part & Tisane & 1 \\
\hline Verbenaceae & $\begin{array}{l}\text { Lippia triphylla } \\
\text { (L'Hér.) O. Kuntze } \\
\text { (BCN29886) }\end{array}$ & Marialluïsa & $\begin{array}{l}\text { Digestive. emmenagogue. for } \\
\text { headache. laxative. not } \\
\text { reported. postpartum } \\
\text { coadjuvant }^{\mathrm{a}} \text {. stomachic }\end{array}$ & Leaf & $\begin{array}{l}\text { Decoction. emulsion. } \\
\text { tisane }\end{array}$ & 21 \\
\hline Violaceae & $\begin{array}{l}\text { Viola alba Besser } \\
\text { (BCN27286) }\end{array}$ & Viola. Violeta & For the influenza & Flower & Tisane & 1 \\
\hline Vitaceae & $\begin{array}{l}\text { Vitis vinifera L. } \\
\text { (BCN29972) }\end{array}$ & $\begin{array}{l}\text { Raïm (fruit). Sarment. Vi } \\
\text { (elaborated product). Vinagre } \\
\text { (elaborated product). Vinya }\end{array}$ & $\begin{array}{l}\text { Analgesic. anticholesterolemic. } \\
\text { antieritematous. antipyretic. } \text { antitussive }{ }^{\mathrm{a}} \text {. for abscesses. for } \\
\text { blood disorders. for stings. } \\
\text { partum coadjuvant }\end{array}$ & Fruit. leaf & $\begin{array}{l}\text { Bath. collutorium. direct } \\
\text { use. medicinal wine. } \\
\text { poultice. tisane }\end{array}$ & 14 \\
\hline
\end{tabular}

${ }^{a}$ Ethnoveterinary

bHuman medicine and ethnoveterinary. No superscripted letter: human medicine

${ }^{c}$ In our country. most coffee industrial presentations are based on C. arabica. the other taxa. such as C. canephora Pierre ex A.Froehner and C. liberica Hiern being clearly minority

because of the medicinal importance of Sambucus nigra. Although Olea europaea, the most relevant species of the Oleaceae, presents medicinal uses as antihypertensive and antihelminthic among others, its high number of use reports is mainly due to the properties of its fruit's oil as an excipient. One of the 20 top medicinal plants,
Hypericum perforatum, is illustrated, prepared for use, in Fig. 2.

Our informants referred 50 (36.5\%) allocthonous taxa with medicinal uses. This high percentage of allocthonous taxa in the western Gironès is an evidence of both the acculturation process and global market influence.

Table 3 List of the 20 most cited species, representing $61.12 \%$ of use reports

\begin{tabular}{|c|c|c|c|c|}
\hline Taxon & Herbarium voucher & Family & UR & $\%$ \\
\hline Sambucus nigra L. & BCN113595 & Adoxaceae & 81 & 8.54 \\
\hline Thymus vulgaris $\mathrm{L}$. & BCN113590 & Lamiaceae & 78 & 8.22 \\
\hline Matricaria recutita $\mathrm{L}$. & BCN113594 & Asteraceae & 46 & 4.85 \\
\hline Olea europaea L. & BCN29898 & Oleaceae & 41 & 4.32 \\
\hline Ruta chalepensis L. & BCN29940 & Rutaceae & 38 & 4.00 \\
\hline Pinus halepensis Mill. & BCN113592 & Pinaceae & 33 & 3.48 \\
\hline Hypericum perforatum L. & BCN113597 & Hypericaceae & 32 & 3.37 \\
\hline Allium sativum $\mathrm{L}$. & BCN29832 & Amaryllidaceae & 29 & 3.06 \\
\hline Mentha pulegium L. & BCN113598 & Lamiaceae & 24 & 2.53 \\
\hline Lippia triphylla (L’Hér.) Kuntze & BCN29886 & Verbenaceae & 21 & 2.21 \\
\hline Zea mays $\mathrm{L}$. & BCN29830 & Poaceae & 20 & 2.11 \\
\hline Eucalyptus globulus Labill. & BCN29696 & Myrtaceae & 18 & 1.90 \\
\hline Asperula cynanchica L. & BCN29634 & Rubiaceae & 16 & 1.69 \\
\hline Cydonia oblonga Mill. & BCN46849 & Rosaceae & 15 & 1.58 \\
\hline Foeniculum vulgare Mill. & BCN26350 & Apiaceae & 15 & 1.58 \\
\hline Linum usitatissimum L. & BCN47281 & Linaceae & 15 & 1.58 \\
\hline Parietaria officinalis L. & BCN113715 & Urticaceae & 15 & 1.58 \\
\hline Ramonda myconi (L.) Rchb. & BCN46088 & Gesneriaceae & 15 & 1.58 \\
\hline Rosmarinus officinalis L. & BCN113599 & Lamiaceae & 14 & 1.48 \\
\hline Triticum aestivum L. & BCN29963 & Poaceae & 14 & 1.48 \\
\hline
\end{tabular}


About two-thirds (32) of allochthonous plants recorded are archaeophytes, and only ca. one-third are neophytes (15) or plants not present in the territory and purchased through commerce (three). This nuances the effect of globalization, which is, nevertheless, significant, with 18 out of 137 taxa (13.1\%) having been introduced in relatively recent times. In addition, some taxa that could be classified as archaeophytes, since their expansion is not related to the Columbian exchange [67], have been recently introduced, in fact, in popular medicine. This is the case of Aloe vera (and other species of the genus used for similar purposes), which could have been introduced as ornamental (and in some cases escaped to the wild) in rather remote times, but are used as medicinal only in recent times; in some cases surely by direct essay in plants cultivated as ornamental and in other cases probably influenced by commercial products based on these plants. In any case, the presence of so-called exotic (allochthonous) plants in European folk medicine would undoubtedly be a good subject for further studies.

The results from the most reported parts are concordant with other Catalan language areas $[5,6,12]$, the aerial part being the most cited (306 UR; 32.24\%), including young aerial, sterile aerial, flowering aerial, and fructified aerial parts; followed by flowers and inflorescences (213; $22.44 \%)$, and fronds or leaves $(147 ; 15.49 \%)$.

A total of 101 types of medicinal uses have been compiled, in which anticatarrhal (59 UR; 6.22\%) and stomachic $(58 ; 6.11 \%)$ are the most frequent. Conversely, grouped by organic systems disorders, the first positions are exchanged, i.e., digestive system disorders occupy the first position, followed by respiratory system disorders (Fig. 3). The seven first histograms in this figure, altogether accounting for almost three-quarters (73.2\%) of medicinal uses, basically represent the kind of remedies mostly used in pharmaceutical ethnobotany or folk medicine and in phytotherapy in general, importantly focused on chronic and usually not very severe illnesses [50,68,69]. This does not at all preclude ethnobotany from being a solid basis for drug development, as already stated by Chadwick \& Marsh [70] and recently confirmed, for instance, with the application of artemisinin and derivatives as the most important antimalarial drugs [71]. In this respect, some of the uses recorded in the present study, apart from those addressing mild ailments-which are also important for everyday lifecould be worthy of further research related to the development of drugs against cardiovascular, mental or immune system disorders, or as a cancer preventive.

The mode of preparation is not very variable. With just two preparation forms, we collect almost $50 \%$ of reports. Tisane, including infusion and decoction, is the most commonly used form, reported 324 times, and represents $34.14 \%$ of forms, followed by remedies without pharmaceutical form $(146 ; 15.38 \%)$.

The number of medicinal plants per informant (MP/I) is 2.40, comparable to the results obtained in Castelló and Ripollès, but lower than in Guilleries (Table 1). This is due to the fact that the number of medicinal plants cited does not increase proportionally to the number of informants but, as the sample grows, the citation of new species becomes less and less frequent. In the same direction, the average of citations for plants does not grow when we increase the number of informants, but tends to stabilize [72].

The number of medicinal plants per inhabitant is slightly higher value than neighboring areas such as Cerdanya or Ripollès and lower than Alt Empordà (Table 1). The number of medicinal plants per $\mathrm{km}^{2}\left(\mathrm{MP} / \mathrm{km}^{2}\right)$ is 0.73, much bigger than those calculated in Cerdanya, Alt Empordà, les Guilleries, Ripollès, and Montseny. We believe that these data are useful to compare territories,

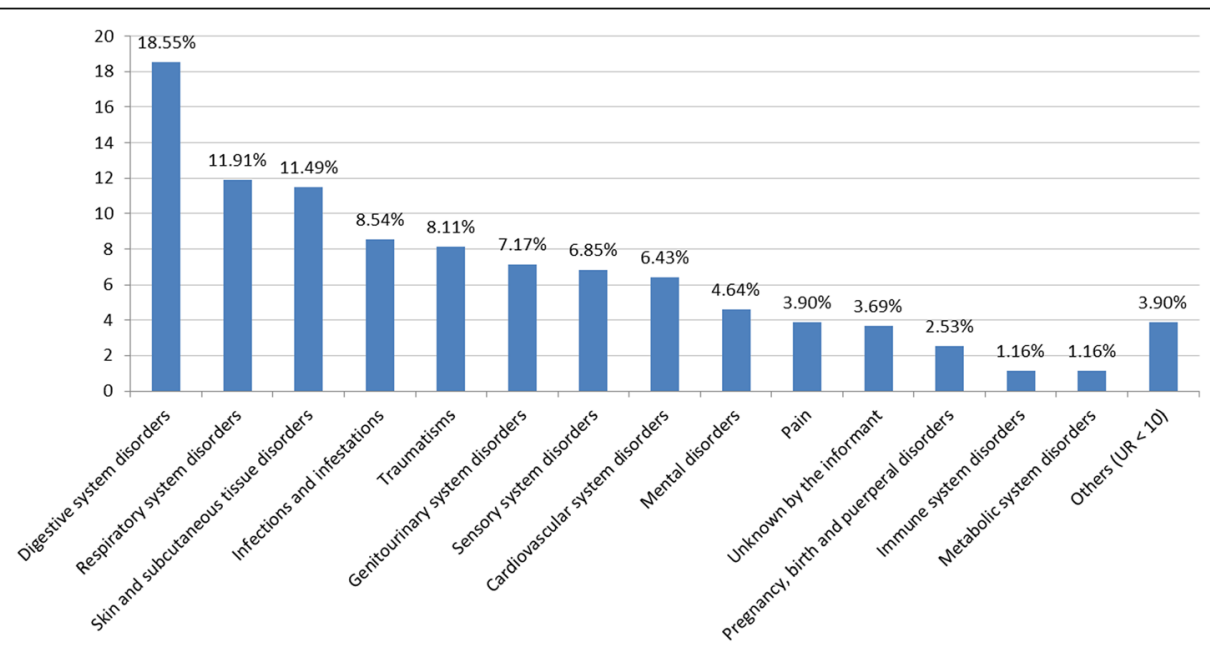

Fig. 3 Organic systems disorders, in percentage, quoted in Gironès 
but it has to be taken into account that they do not basically depend on the informant sampling but on the total population or extension of each studied area.

This study has also inventoried 55 plant mixtures with 80 different taxa; the 3 most reported species are Thymus vulgaris with 16 citations and present in a $29.09 \%$ of mixtures, Triticum aestivum (14; 25.45\%) and Rosmarinus officinalis $(13 ; 23.64 \%)$. These mixtures were used to treat 28 diseases, and if we group the diseases by organic system disorders, respiratory, and pregnancy, birth and puerperal disorders are ranked in first position, both with $20 \%$ of mixtures. The first disorder is associated with human use and the second one with veterinary use as a postpartum coadjuvant, results that in accordance with those mentioned in Gras et al. [52]. The number of species per mixture varies largely from 1 mixture to another, ranging from 2 to 28 species per mixture and the average number of taxa or ingredients is 3.31 . The mixture with the highest number of taxa is a poultice for pneumonia treatment.

The informant consensus factor $\left(\mathrm{F}_{\mathrm{IC}}\right)$ for mixtures data is 0.56 , a lower value than in the recent before-mentioned study, devoted to plant mixtures in two Catalan territories [52]. The index of taxon usefulness in mixtures (ITUM), calculated for all taxa with more than three use reports in mixtures, is lower than 0.50 in almost all cases, except in Triticum aestivum (0.50) and Lavandula stoechas (0.67). The results show that there is not a trend of exclusiveness of taxa use in mixtures, but taxa used in mixtures are also employed alone.

\section{Food uses}

Food uses are the most reported by the informants in this area: 224 taxa belonging to 66 families constitute an important dataset, accumulating 1933 use reports. Six of these taxa have only been determined at the genus level, and from the remaining 218 ones, 3 of them have infraspecific categories. All of these results show a clear difference with other studied areas, where the medicinal uses predominate over food uses $[6,10,58]$. We do not have a clear hypothesis that explains this surprising result, but although some biases could have occurred in the interviewing process, the importance of crops and the proximity of the markets are two factors that need to be taken into account in this area.

Concerning the destination, a $70.67 \%$ of these taxa are used in human food, a $28.30 \%$ in animal feed, and there is no information for the remaining $1.03 \%$. The mean of food taxa cited by informant is 3.42 for human food and 1.42 for animal feed.

The most reported families for human food consumption (Table 4) are Lamiaceae (272 UR), Rosaceae (174 UR), and Asteraceae (126 UR). These are families well represented in the Mediterranean flora as in the case of medicinal plants, and some of them comprising relevant categories for human food such as aromatic plants and fruit trees. The rare families concerning both the number of species and number of use reports usually correspond to taxa acquired through commerce. The five species that have received more citations are Mentha spicata (50 UR), Reichardia picroides (37 UR), Laurus nobilis (36 UR), Arbutus unedo (35 UR), and Rubus ulmifolius (34 UR). These are wild species, but Laurus nobilis is also very frequently cultivated near houses. Additionally, Reichardia picroides, one of the most well-known food (and also feed) plants in the Catalan linguistic area $[34,58]$ and in other Mediterranean areas $[32,73]$, is a wild species, which, as reported by Maggini et al. [74] in a study in Tuscany involving cultivation of several ecotypes, seems to be a promising vegetable crop, with nutraceutical properties. Further research in this sense in different areas would be desirable to confirm this point.

Fruit (including fructification in the gymnosperms) and infructescence are the most used plant parts (29.28\%), followed by aerial part, including the whole plant sometimes used in the same way by our informants (27.6\%), flower and inflorescence (17.28\%), and leaf (14.2\%). Regarding the preparation forms, two of them are nearly tied at the top of the ranking; the first one, the beverage prepared with alcohol (39.02\%) for the importance of traditional liqueur called ratafia, and the second one, the raw plant (20.79\%), which is not really a form of preparation because it implies the direct use of raw material. The plant cooked in several ways (16.76\%) and condiments $(16.25 \%)$ are the two categories that follow them.

As an example of alcoholic beverages, apart from punctual quotations, we collected 6 complete receipts of ratafia in the studied area, the most diverse in terms of plant taxa comprising 76 species. All of them (including the young Juglans regia fruits with a few incisions made) are put together in maceration in a big glass bottle with an anisate alcohol, conserved typically 40 days in an external part of the house, and then filtered and, if necessary, corrected in sugar. After this, and with a final graduation around $23^{\circ}$, it may be consumed as a pleasure and medicinal liqueur. Some of the plants ready to prepare ratafia are shown in Fig. 2.

To illustrate a few food elaborations, Urtica dioica, usually known as a medicinal plant-also employed as such in the studied area-appears as one of the wild plants with more variation: it may be scalded and then either seasoned and consumed as a vegetable or prepared in omelet, and it may be boiled and eaten in soup. Sambucus nigra flowers are consumed in a very common way in northeast Catalonian areas [10, 
Table 4 Food plants reported in the studied area

\begin{tabular}{|c|c|c|c|c|c|c|}
\hline Family & Taxon (voucher) & Catalan vernacular names & Preparation & Used part & $\begin{array}{l}\text { Wild }(\mathrm{W}) / \\
\text { cultivated }(\mathrm{C})\end{array}$ & $\begin{array}{l}\text { Use } \\
\text { reports }\end{array}$ \\
\hline Adoxaceae & $\begin{array}{l}\text { Sambucus nigra L. } \\
\text { (BCN113595) }\end{array}$ & Sabuquer. Saüc. Saüquer & $\begin{array}{l}\text { Alcoholic beverage made } \\
\text { with wine. Cooked in oil. } \\
\text { Cooked in wine. Cooked } \\
\text { with sugar. High-grade } \\
\text { alcoholic beverage }\end{array}$ & Fruit. Inflorescence & W & 15 \\
\hline Amaranthaceae & $\begin{array}{l}\text { Beta vulgaris L. } \\
\text { subsp. vulgaris var. } \\
\text { crassa (Alef.) Helm } \\
\text { (BCN50761) }\end{array}$ & $\begin{array}{l}\text { Bleda. Polpa (elaborated product). } \\
\text { Remolatxa. Sucre (elaborated } \\
\text { product). Sucre candi (elaborated } \\
\text { product) }\end{array}$ & $\begin{array}{l}\text { Air dried. Alcoholic } \\
\text { beverage made with } \\
\text { wine. High-grade alco- } \\
\text { holic beverage }\end{array}$ & Root & C & 9 \\
\hline \multirow[t]{3}{*}{ Amaryllidaceae } & $\begin{array}{l}\text { Allium cepa L. } \\
\text { (BCN28655) }\end{array}$ & Ceba & $\begin{array}{l}\text { Boiled in water. Cooked. } \\
\text { Raw }\end{array}$ & Bulb & C & 4 \\
\hline & $\begin{array}{l}\text { Allium porrum L. } \\
\text { (BCN28791) }\end{array}$ & Porro & Condiment & Leaf & C & 2 \\
\hline & $\begin{array}{l}\text { Allium sativum L. } \\
\text { (BCN29832) }\end{array}$ & All & $\begin{array}{l}\text { Boiled in water. } \\
\text { Condiment. Cooked in } \\
\text { oil. Raw }\end{array}$ & Bulb & C & 10 \\
\hline \multirow[t]{8}{*}{ Apiaceae } & $\begin{array}{l}\text { Apium nodiflorum } \\
\text { (L.) Lag. } \\
\text { (BCN113736) }\end{array}$ & Créixec. Creixen & Raw & Aerial part & W & 4 \\
\hline & $\begin{array}{l}\text { Carum carvi L. } \\
\text { (BCN29642) }\end{array}$ & Comí & $\begin{array}{l}\text { High-grade alcoholic } \\
\text { beverage }\end{array}$ & Flower & W & 1 \\
\hline & $\begin{array}{l}\text { Cuminum cyminum } \\
\text { L. (BCN-E-194) }\end{array}$ & Comí & $\begin{array}{l}\text { High-grade alcoholic } \\
\text { beverage }\end{array}$ & Seed & W & 1 \\
\hline & $\begin{array}{l}\text { Daucus carota L. } \\
\text { subsp. sativus } \\
\text { (Hoffm.) Arcang. } \\
\text { (BCN46847) }\end{array}$ & Pastanaga & Boiled in water & Root & C & 1 \\
\hline & $\begin{array}{l}\text { Eryngium campestre } \\
\text { L. (BCN31274) }\end{array}$ & Espinacal & $\begin{array}{l}\text { High-grade alcoholic } \\
\text { beverage }\end{array}$ & Flower & W & 1 \\
\hline & $\begin{array}{l}\text { Foeniculum vulgare } \\
\text { Mill. (BCN26350) }\end{array}$ & Fonoll & $\begin{array}{l}\text { Boiled in water. } \\
\text { Condiment. High-grade } \\
\text { alcoholic beverage }\end{array}$ & $\begin{array}{l}\text { Aerial part. } \\
\text { Inflorescence }\end{array}$ & W & 27 \\
\hline & $\begin{array}{l}\text { Petroselinum } \\
\text { crispum (Mill.) Hill } \\
\text { (BCN29905) }\end{array}$ & Julivert & Condiment & Leaf & C & 4 \\
\hline & $\begin{array}{l}\text { Pimpinella anisum L. } \\
\text { (BCN47278) }\end{array}$ & Anís verd. Matafaluga & $\begin{array}{l}\text { Condiment. High-grade } \\
\text { alcoholic beverage }\end{array}$ & Fruit & C & 6 \\
\hline Apocynaceae & $\begin{array}{l}\text { Vinca major L. } \\
\text { (BCN25039) }\end{array}$ & Vinca. Vincapervinca & $\begin{array}{l}\text { High-grade alcoholic } \\
\text { beverage }\end{array}$ & Flower & W & 2 \\
\hline \multirow[t]{2}{*}{ Asparagaceae } & $\begin{array}{l}\text { Aphyllanthes } \\
\text { monspeliensis L. } \\
\text { (BCN29627) }\end{array}$ & Llonsa. Pa de cucut & Raw & Aerial part. Flower & W & 3 \\
\hline & $\begin{array}{l}\text { Asparagus acutifolius } \\
\text { L. (BCN29976) }\end{array}$ & Espàrgol. Esparreguera. Espàrrec & $\begin{array}{l}\text { Cooked in oil. High-grade } \\
\text { alcoholic beverage }\end{array}$ & Leaf. Young shoot & W & 12 \\
\hline Aspleniaceae & $\begin{array}{l}\text { Asplenium } \\
\text { adiantum-nigrum L. } \\
\text { (BCN113596) }\end{array}$ & Fulleta & $\begin{array}{l}\text { High-grade alcoholic } \\
\text { beverage }\end{array}$ & Frond & W & 1 \\
\hline \multirow[t]{4}{*}{ Asteraceae } & $\begin{array}{l}\text { Achillea ageratum L. } \\
\text { (BCN113701) }\end{array}$ & Herba del fàstic & $\begin{array}{l}\text { High-grade alcoholic } \\
\text { beverage }\end{array}$ & Inflorescence & W & 1 \\
\hline & $\begin{array}{l}\text { Achillea millefolium } \\
\text { L. (BCN113708) }\end{array}$ & Cordonet. Herba de les milfulles & $\begin{array}{l}\text { High-grade alcoholic } \\
\text { beverage }\end{array}$ & Aerial part & W & 1 \\
\hline & $\begin{array}{l}\text { Arctium minus } \\
\text { Bernh. (BCN113727) }\end{array}$ & - & Boiled in water & Stem & W & 1 \\
\hline & $\begin{array}{l}\text { Artemisia } \\
\text { abrotanum L. }\end{array}$ & Broida & Condiment & Leaf & W & 1 \\
\hline
\end{tabular}


Table 4 Food plants reported in the studied area (Continued)

\begin{tabular}{|c|c|c|c|c|c|c|}
\hline Family & Taxon (voucher) & Catalan vernacular names & Preparation & Used part & $\begin{array}{l}\text { Wild }(\mathrm{W}) / \\
\text { cultivated }(\mathrm{C})\end{array}$ & $\begin{array}{l}\text { Use } \\
\text { reports }\end{array}$ \\
\hline & $\begin{array}{l}\text { Artemisia } \\
\text { absinthium L. } \\
\text { (BCN29837) }\end{array}$ & Artemisa. Donzell & $\begin{array}{l}\text { High-grade alcoholic } \\
\text { beverage }\end{array}$ & Aerial part & W & 1 \\
\hline & $\begin{array}{l}\text { Bellis perennis L. } \\
\text { (BCN31264) }\end{array}$ & Margaridoia & Raw & Leaf & W & 2 \\
\hline & $\begin{array}{l}\text { Calendula officinalis } \\
\text { L. (BCN29977) }\end{array}$ & Calèndula & $\begin{array}{l}\text { High-grade alcoholic } \\
\text { beverage. Raw }\end{array}$ & Inflorescence & C & 2 \\
\hline & $\begin{array}{l}\text { Centaurea aspera L. } \\
\text { (BCN113579) }\end{array}$ & $\begin{array}{l}\text { Caps de burro. Flor del sucre. } \\
\text { Travalera }\end{array}$ & $\begin{array}{l}\text { High-grade alcoholic } \\
\text { beverage }\end{array}$ & Inflorescence & W & 2 \\
\hline & $\begin{array}{l}\text { Chondrilla juncea L. } \\
\text { (BCN29852) }\end{array}$ & Màstec & Preserved in vinegar. Raw & Leaf & W & 29 \\
\hline & $\begin{array}{l}\text { Cichorium endivia } \mathrm{L} . \\
\text { (BCN46854) }\end{array}$ & Escarola & Condiment & Leaf & C & 1 \\
\hline & $\begin{array}{l}\text { Cichorium intybus L. } \\
\text { (BCN29660) }\end{array}$ & Cama-roja. Xicoia. Xicoina. Xicòria & Raw & Leaf & W & 6 \\
\hline & $\begin{array}{l}\text { Cynara cardunculus } \\
\text { L. (BCN-E-192) }\end{array}$ & $\begin{array}{l}\text { Herba presonera. Presó. Presonera. } \\
\text { Presora }\end{array}$ & Condiment & Inflorescence & C & 18 \\
\hline & $\begin{array}{l}\text { Helichrysum } \\
\text { stoechas (L.) } \\
\text { Moench } \\
\text { (BCN29872) }\end{array}$ & Mançanilla. Sempreviva & $\begin{array}{l}\text { High-grade alcoholic } \\
\text { beverage }\end{array}$ & $\begin{array}{l}\text { Aerial part. } \\
\text { Inflorescence }\end{array}$ & W & 3 \\
\hline & $\begin{array}{l}\text { Matricaria recutita L. } \\
\text { (BCN113594) }\end{array}$ & Camamilla. Camamilla romana & $\begin{array}{l}\text { High-grade alcoholic } \\
\text { beverage }\end{array}$ & $\begin{array}{l}\text { Aerial part. } \\
\text { Inflorescence }\end{array}$ & W & 11 \\
\hline & $\begin{array}{l}\text { Pallenis spinosa (L.) } \\
\text { Cass. (BCN31291) }\end{array}$ & Mala mare & $\begin{array}{l}\text { High-grade alcoholic } \\
\text { beverage }\end{array}$ & Inflorescence & W & 2 \\
\hline & $\begin{array}{l}\text { Reichardia picroides } \\
\text { (L.) Roth } \\
\text { (BCN113704) }\end{array}$ & Cosconilla & $\begin{array}{l}\text { High-grade alcoholic } \\
\text { beverage. Raw }\end{array}$ & Leaf & W & 37 \\
\hline & $\begin{array}{l}\text { Santolina } \\
\text { chamaecyparissus L. } \\
\text { (BCN113709) }\end{array}$ & Espernallac. Santolina & $\begin{array}{l}\text { High-grade alcoholic } \\
\text { beverage. Milk-based } \\
\text { beverage }\end{array}$ & Inflorescence & W & 2 \\
\hline & $\begin{array}{l}\text { Silybum marianum } \\
\text { (L.) Gaertn. } \\
\text { (BCN29958) }\end{array}$ & Card & Boiled in water & Stem & W & 1 \\
\hline & Sonchus sp. & Llepsó. Lletissó. Llistó & Raw & Aerial part & W & 1 \\
\hline & $\begin{array}{l}\text { Stevia rebaudiana } \\
\text { (Bertoni) Bertoni } \\
\text { (BCN30644) }\end{array}$ & Estèvia & Not reported & Leaf & C & 1 \\
\hline & $\begin{array}{l}\text { Taraxacum officinale } \\
\text { Weber in Wiggers } \\
\text { (BCN25948) }\end{array}$ & Dent de lleó. Xicoia & $\begin{array}{l}\text { Beverage made with } \\
\text { water. High-grade alco- } \\
\text { holic beverage. Raw }\end{array}$ & $\begin{array}{l}\text { Leaf. Not } \\
\text { reported. Root }\end{array}$ & W & 3 \\
\hline Betulaceae & $\begin{array}{l}\text { Corylus avellana L. } \\
\text { (BCN29831) }\end{array}$ & $\begin{array}{l}\text { Avellana (fruit). Avellana del queixal } \\
\text { (fruit). Avellaner. Avellaner negret }\end{array}$ & Cooked. Raw & Fruit & W & 7 \\
\hline \multirow[t]{4}{*}{ Brassicaceae } & $\begin{array}{l}\text { Brassica napus } \mathrm{L} \text {. } \\
\text { (BCN46856) }\end{array}$ & Nap. Nap de bou. Nap del camp & Boiled in water & Root & $C$ & 1 \\
\hline & $\begin{array}{l}\text { Brassica oleracea L. } \\
\text { subsp. oleracea } \\
\text { (BCN32181) }\end{array}$ & Bròquil. Col. Col aloma & $\begin{array}{l}\text { Preserved in salt and } \\
\text { water }\end{array}$ & Leaf & C & 1 \\
\hline & $\begin{array}{l}\text { Capsella bursa- } \\
\text { pastoris (L.) Medic. } \\
\text { (BCN46079) }\end{array}$ & Bossa de pastor. Caps blancs & $\begin{array}{l}\text { High-grade alcoholic } \\
\text { beverage. Raw }\end{array}$ & Aerial part & W & 2 \\
\hline & $\begin{array}{l}\text { Raphanus } \\
\text { raphanistrum L. } \\
\text { subsp. sativus (L.) } \\
\text { Domin (BCN49860) }\end{array}$ & Rave & Raw & Stem & C & 1 \\
\hline
\end{tabular}


Table 4 Food plants reported in the studied area (Continued)

\begin{tabular}{|c|c|c|c|c|c|c|}
\hline Family & Taxon (voucher) & Catalan vernacular names & Preparation & Used part & $\begin{array}{l}\text { Wild (W)/ } \\
\text { cultivated (C) }\end{array}$ & $\begin{array}{l}\text { Use } \\
\text { reports }\end{array}$ \\
\hline & $\begin{array}{l}\text { Rorippa nasturtium- } \\
\text { aquaticum (L.) } \\
\text { Hayek subsp. } \\
\text { nasturtium- } \\
\text { aquaticum } \\
\text { (BCN29771) }\end{array}$ & Créixec. Creixen & Raw & Aerial part & W & 6 \\
\hline Cactaceae & $\begin{array}{l}\text { Opuntia maxima } \\
\text { Mill. (BCN46078) }\end{array}$ & Figuera de moro & Condiment & Leaf & C & 1 \\
\hline Campanulaceae & $\begin{array}{l}\text { Campanula } \\
\text { rapunculus L. } \\
\text { (BCN50763) }\end{array}$ & Repunxó & Raw & $\begin{array}{l}\text { Leaf. Root. Whole } \\
\text { plant }\end{array}$ & W & 22 \\
\hline Cannabaceae & $\begin{array}{l}\text { Celtis australis L. } \\
\text { (BCN29845) }\end{array}$ & Lledó (fruit). Lledoner & $\begin{array}{l}\text { High-grade alcoholic } \\
\text { beverage. Raw }\end{array}$ & Fruit & C & 15 \\
\hline \multirow[t]{3}{*}{ Caprifoliaceae } & $\begin{array}{l}\text { Lonicera implexa Ait. } \\
\text { (BCN113802) }\end{array}$ & Lligabosc. Mareselva. Xuclamel & $\begin{array}{l}\text { High-grade alcoholic } \\
\text { beverage. Raw }\end{array}$ & Aerial part. Flower & W & 13 \\
\hline & $\begin{array}{l}\text { Scabiosa } \\
\text { atropurpurea L. } \\
\text { (BCN29947) }\end{array}$ & Escabiosa & $\begin{array}{l}\text { High-grade alcoholic } \\
\text { beverage }\end{array}$ & Flower & W & 5 \\
\hline & $\begin{array}{l}\text { Valerianella locusta } \\
\text { (L.) Laterrade } \\
\text { (BCN49861) }\end{array}$ & Margarideta. Margaridoia. Marieta & Raw & Leaf & w & 5 \\
\hline \multirow[t]{4}{*}{ Caryophyllaceae } & $\begin{array}{l}\text { Dianthus } \\
\text { caryophyllus L. } \\
\text { (BCN31272) }\end{array}$ & Clavell. Clavell domèstic & $\begin{array}{l}\text { High-grade alcoholic } \\
\text { beverage }\end{array}$ & Flower & C & 4 \\
\hline & $\begin{array}{l}\text { Dianthus seguieri } \\
\text { Vill. (BCN113734) }\end{array}$ & Clavell de pastor & $\begin{array}{l}\text { High-grade alcoholic } \\
\text { beverage }\end{array}$ & Flower & W & 1 \\
\hline & $\begin{array}{l}\text { Herniaria glabra L. } \\
\text { (BCN113577) }\end{array}$ & Herba de les mil granes. Mil granes & $\begin{array}{l}\text { High-grade alcoholic } \\
\text { beverage }\end{array}$ & Aerial part & W & 5 \\
\hline & $\begin{array}{l}\text { Silene vulgaris } \\
\text { (Moench) Garcke } \\
\text { (BCN29948) }\end{array}$ & Culivells & Boiled in water & Leaf & W & 5 \\
\hline Cistaceae & $\begin{array}{l}\text { Cistus salviifolius L. } \\
\text { (BCN36767) }\end{array}$ & Estepa. Mòdega & $\begin{array}{l}\text { High-grade alcoholic } \\
\text { beverage }\end{array}$ & Flower & W & 2 \\
\hline Clusiaceae & $\begin{array}{l}\text { Hypericum } \\
\text { perforatum L. } \\
\text { (BCN113597) }\end{array}$ & $\begin{array}{l}\text { Flor de Sant Joan. Herba de cop. } \\
\text { Herba de Sant Joan }\end{array}$ & $\begin{array}{l}\text { High-grade alcoholic } \\
\text { beverage }\end{array}$ & Aerial part. Flower & W & 12 \\
\hline Convolvulaceae & $\begin{array}{l}\text { Convolvulus arvensis } \\
\text { L. (BCN29663) }\end{array}$ & Corretjola & $\begin{array}{l}\text { High-grade alcoholic } \\
\text { beverage }\end{array}$ & Flower & W & 2 \\
\hline Crassulaceae & $\begin{array}{l}\text { Sempervivum } \\
\text { tectorum L. } \\
\text { (BCN26780) }\end{array}$ & Matifoc & $\begin{array}{l}\text { High-grade alcoholic } \\
\text { beverage }\end{array}$ & Leaf & W & 1 \\
\hline \multirow[t]{5}{*}{ Cucurbitaceae } & $\begin{array}{l}\text { Citrullus lanatus } \\
\text { (Thunb.) Matsumara } \\
\text { et Nakai (BCN29662) }\end{array}$ & Síndria. Xíndria & Cooked with sugar & Epicarp. Fruit & C & 2 \\
\hline & $\begin{array}{l}\text { Cucumis melo L. } \\
\text { (BCN46851) }\end{array}$ & Meló (fruit) & Cooked with sugar. Raw & Fruit & $C$ & 6 \\
\hline & $\begin{array}{l}\text { Cucurbita ficifolia } \\
\text { C.D.Bouché in Verh. } \\
\text { (BCN29980) }\end{array}$ & Carabassa de cabell d'àngel & Cooked with sugar & Fruit & $C$ & 1 \\
\hline & $\begin{array}{l}\text { Cucurbita pepo L. } \\
\text { var. oblonga Link } \\
\text { (BCN29859) }\end{array}$ & Carabassó (fruit) & $\begin{array}{l}\text { Boiled in water. Cooked } \\
\text { in oil }\end{array}$ & Flower. Fruit & $C$ & 4 \\
\hline & $\begin{array}{l}\text { Cucurbita pepo L. } \\
\text { var. pepo } \\
\text { (BCN49858) }\end{array}$ & $\begin{array}{l}\text { Carbassa (fruit). Carbassera. Rabequet } \\
\text { (fruit) }\end{array}$ & Boiled in water & Fruit & $C$ & 2 \\
\hline Cupressaceae & Juniperus communis & Ginebre. Ginebró & Air dried. Alcoholic & Fruit & W & 12 \\
\hline
\end{tabular}


Table 4 Food plants reported in the studied area (Continued)

\begin{tabular}{|c|c|c|c|c|c|c|}
\hline Family & Taxon (voucher) & Catalan vernacular names & Preparation & Used part & $\begin{array}{l}\text { Wild (W)/ } \\
\text { cultivated (C) }\end{array}$ & $\begin{array}{l}\text { Use } \\
\text { reports }\end{array}$ \\
\hline & L. (BCN113589) & & $\begin{array}{l}\text { beverage made with } \\
\text { wine. Condiment. High- } \\
\text { grade alcoholic beverage }\end{array}$ & & & \\
\hline & $\begin{array}{l}\text { Juniperus oxycedrus } \\
\text { L. (BCN29879) }\end{array}$ & Càdec & $\begin{array}{l}\text { High-grade alcoholic } \\
\text { beverage }\end{array}$ & Fruit & W & 1 \\
\hline \multirow[t]{3}{*}{ Equisetaceae } & $\begin{array}{l}\text { Equisetum arvense } \mathrm{L} . \\
\text { (BCN24767) }\end{array}$ & Cua de cavall. Sangnua & $\begin{array}{l}\text { High-grade alcoholic } \\
\text { beverage }\end{array}$ & Aerial part & W & 1 \\
\hline & Equisetum sp. & Cua de cavall. Sangnua & $\begin{array}{l}\text { High-grade alcoholic } \\
\text { beverage }\end{array}$ & Aerial part & W & 4 \\
\hline & $\begin{array}{l}\text { Equisetum telmateia } \\
\text { Ehrh. (BCN113581) }\end{array}$ & Cua de cavall. Sangnua & $\begin{array}{l}\text { High-grade alcoholic } \\
\text { beverage }\end{array}$ & Aerial part & W & 3 \\
\hline \multirow[t]{3}{*}{ Ericaceae } & $\begin{array}{l}\text { Arbutus unedo L. } \\
\text { (BCN29836) }\end{array}$ & $\begin{array}{l}\text { Arboç. Bola d'arboç (fruit). Cirera } \\
\text { d'arboç (fruit). Cirerer d'arboç }\end{array}$ & $\begin{array}{l}\text { Cooked with sugar. High- } \\
\text { grade alcoholic beverage. } \\
\text { Raw }\end{array}$ & Fruit & W & 35 \\
\hline & $\begin{array}{l}\text { Calluna vulgaris (L.) } \\
\text { Hull (BCN113722) }\end{array}$ & Bronsa. Bronsó & Raw & Flower & W & 1 \\
\hline & $\begin{array}{l}\text { Erica arborea L. } \\
\text { (BCN113593) }\end{array}$ & $\begin{array}{l}\text { Bruc. Bruc boal. Bruc bord. Bruc d'ull } \\
\text { de bou. Bruc de bou. Bruc de llei }\end{array}$ & $\begin{array}{l}\text { High-grade alcoholic } \\
\text { beverage }\end{array}$ & Flower & W & 3 \\
\hline Euphorbiaceae & Euphorbia sp. & Llet de Tereses. Lletdetresa & Condiment & Latex & W & 1 \\
\hline \multirow[t]{11}{*}{ Fabaceae } & $\begin{array}{l}\text { Acacia dealbata } \\
\text { Link. (BCN29973) }\end{array}$ & Mimosa & $\begin{array}{l}\text { High-grade alcoholic } \\
\text { beverage }\end{array}$ & Flower & C & 2 \\
\hline & $\begin{array}{l}\text { Ceratonia siliqua L. } \\
\text { (BCN32177) }\end{array}$ & Garrofa (fruit) & Air dried & Fruit & $C$ & 5 \\
\hline & $\begin{array}{l}\text { Glycyrrhiza glabra L. } \\
\text { (BCN47276) }\end{array}$ & Regalíssia & $\begin{array}{l}\text { High-grade alcoholic } \\
\text { beverage }\end{array}$ & Root & W & 2 \\
\hline & $\begin{array}{l}\text { Lathyrus latifolius L. } \\
\text { (BCN29712) }\end{array}$ & Pèsol bord & $\begin{array}{l}\text { Boiled with water. High- } \\
\text { grade alcoholic beverage }\end{array}$ & Flower. Fruit & W & 4 \\
\hline & $\begin{array}{l}\text { Onobrychis viciifolia } \\
\text { Scop. (BCN113732) }\end{array}$ & Esparcet. Trepadella & $\begin{array}{l}\text { High-grade alcoholic } \\
\text { beverage }\end{array}$ & Flower & $C$ & 2 \\
\hline & $\begin{array}{l}\text { Phaseolus vulgaris } \mathrm{L} \text {. } \\
\text { (BCN46837) }\end{array}$ & $\begin{array}{l}\text { Fesol de l'ull marrón. Fesol de l'ull } \\
\text { negre. Fesol de Santa Pau. Fesol del } \\
\text { bitxet gros. Fesol de bitxet petit. } \\
\text { Fesol menut. Fesola. Mongeta }\end{array}$ & Boiled in water & Seed & $C$ & 5 \\
\hline & $\begin{array}{l}\text { Robinia } \\
\text { pseudoacacia L. } \\
\text { (BCN31298) }\end{array}$ & Acàcia. Acàcia de jardí & $\begin{array}{l}\text { High-grade alcoholic } \\
\text { beverage }\end{array}$ & Flower & $C$ & 2 \\
\hline & $\begin{array}{l}\text { Spartium junceum L. } \\
\text { (BCN29956) }\end{array}$ & Ginesta & $\begin{array}{l}\text { High-grade alcoholic } \\
\text { beverage }\end{array}$ & Flower & W & 9 \\
\hline & $\begin{array}{l}\text { Trifolium incarnatum } \\
\text { L. (BCN25026) }\end{array}$ & Fenc & $\begin{array}{l}\text { Boiled in water. High- } \\
\text { grade alcoholic beverage }\end{array}$ & Aerial part. Flower & C & 3 \\
\hline & $\begin{array}{l}\text { Vicia faba L. } \\
\text { (BCN46826) }\end{array}$ & Fava & $\begin{array}{l}\text { Boiled in water. Cooked. } \\
\text { Cooked in oil }\end{array}$ & Seed & $C$ & 6 \\
\hline & $\begin{array}{l}\text { Vicia sativa L. } \\
\text { (BCN47746) }\end{array}$ & Veça & Raw & Aerial part & C & 1 \\
\hline \multirow[t]{3}{*}{ Fagaceae } & $\begin{array}{l}\text { Castanea sativa Mill. } \\
\text { (BCN29844) }\end{array}$ & Castanya (fruit). Castanyer & Boiled in water. Cooked & Fruit & $C$ & 9 \\
\hline & $\begin{array}{l}\text { Quercus ilex L. } \\
\text { (BCN113730) }\end{array}$ & Aglà (fruit). Alzina. Aulina. Gla (fruit) & $\begin{array}{l}\text { Cooked. High-grade alco- } \\
\text { holic beverage }\end{array}$ & Flower. Fruit & W & 7 \\
\hline & $\begin{array}{l}\text { Quercus suber L. } \\
\text { (BCN46829) }\end{array}$ & Suro. Alzina surera & Air dried & Fruit & W & 1 \\
\hline Gesneriaceae & $\begin{array}{l}\text { Ramonda myconi } \\
\text { (L.) Reichenb. } \\
\text { (BCN46088) }\end{array}$ & Orella d'os & $\begin{array}{l}\text { High-grade alcoholic } \\
\text { beverage }\end{array}$ & Aerial part. Leaf & W & 4 \\
\hline Hydrangeaceae & Philadelphus & Xeringuilla & High-grade alcoholic & Flower & C & 2 \\
\hline
\end{tabular}


Table 4 Food plants reported in the studied area (Continued)

\begin{tabular}{|c|c|c|c|c|c|c|}
\hline Family & Taxon (voucher) & Catalan vernacular names & Preparation & Used part & $\begin{array}{l}\text { Wild (W)/ } \\
\text { cultivated (C) }\end{array}$ & $\begin{array}{l}\text { Use } \\
\text { reports }\end{array}$ \\
\hline & $\begin{array}{l}\text { coronarius L. } \\
\text { (BCN27261) }\end{array}$ & & beverage & & & \\
\hline Iridaceae & $\begin{array}{l}\text { Crocus sativus L. } \\
\text { (BCN32170) }\end{array}$ & Safrà & Condiment & Styles and stigmas & C & 1 \\
\hline Juglandaceae & $\begin{array}{l}\text { Juglans regia L. } \\
\text { (BCN29877) }\end{array}$ & Noguer. Nou (fruit). Nou verda (fruit) & $\begin{array}{l}\text { Air dried. Alcoholic } \\
\text { beverage made with } \\
\text { wine. Condiment. High- } \\
\text { grade alcoholic beverage }\end{array}$ & Fruit. Leaf & C & 29 \\
\hline \multirow[t]{20}{*}{ Lamiaceae } & $\begin{array}{l}\text { Hyssopus officinalis } \\
\text { L. (BCN29709) }\end{array}$ & Hisop & $\begin{array}{l}\text { High-grade alcoholic } \\
\text { beverage }\end{array}$ & Aerial part. Flower & W & 5 \\
\hline & $\begin{array}{l}\text { Lamium flexuosum } \\
\text { Ten. (BCN26731) }\end{array}$ & Ortiga blanca & $\begin{array}{l}\text { High-grade alcoholic } \\
\text { beverage }\end{array}$ & Flower & W & 2 \\
\hline & $\begin{array}{l}\text { Lavandula dentata } \\
\text { L. (BCN29715) }\end{array}$ & Lavanda & $\begin{array}{l}\text { High-grade alcoholic } \\
\text { beverage }\end{array}$ & Flower & W & 1 \\
\hline & $\begin{array}{l}\text { Lavandula latifolia } \\
\text { Medic. (BCN113740) }\end{array}$ & Barballó. Espígol. Lavanda & $\begin{array}{l}\text { High-grade alcoholic } \\
\text { beverage }\end{array}$ & Aerial part & W & 4 \\
\hline & $\begin{array}{l}\text { Lavandula stoechas } \\
\text { L. (BCN113714) }\end{array}$ & Cap d'ase. Tomanyí & $\begin{array}{l}\text { High-grade alcoholic } \\
\text { beverage }\end{array}$ & Aerial part. Flower & W & 6 \\
\hline & $\begin{array}{l}\text { Melissa officinalis L. } \\
\text { (BCN113713) }\end{array}$ & Melissa. Tarongina & $\begin{array}{l}\text { High-grade alcoholic } \\
\text { beverage }\end{array}$ & Aerial part. Leaf & C & 12 \\
\hline & $\begin{array}{l}\text { Mentha xpiperita L. } \\
\text { (BCN113813) }\end{array}$ & $\begin{array}{l}\text { Menta. Menta de la xocolata. Menta } \\
\text { piperita. Menta romana }\end{array}$ & $\begin{array}{l}\text { Condiment. High-grade } \\
\text { alcoholic beverage }\end{array}$ & Aerial part & C & 15 \\
\hline & $\begin{array}{l}\text { Mentha pulegium L. } \\
\text { (BCN113598) }\end{array}$ & Poliol. Poniol & $\begin{array}{l}\text { High-grade alcoholic } \\
\text { beverage }\end{array}$ & Aerial part. Flower & W & 8 \\
\hline & $\begin{array}{l}\text { Mentha spicata L. } \\
\text { (BCN113812) }\end{array}$ & $\begin{array}{l}\text { Menta. Menta de la sopa. Menta } \\
\text { silvestre. Menta espicata. Menta } \\
\text { verdadera }\end{array}$ & $\begin{array}{l}\text { Boiled with water. } \\
\text { Condiment. High-grade } \\
\text { alcoholic beverage }\end{array}$ & Aerial part & W & 50 \\
\hline & $\begin{array}{l}\text { Mentha suaveolens } \\
\text { Ehrh. (BCN113810) }\end{array}$ & Menta blanca & $\begin{array}{l}\text { High-grade alcoholic } \\
\text { beverage }\end{array}$ & Aerial part & W & 3 \\
\hline & $\begin{array}{l}\text { Nepeta cataria L. } \\
\text { (BCN113798) }\end{array}$ & Gatera. Nepta & $\begin{array}{l}\text { High-grade alcoholic } \\
\text { beverage }\end{array}$ & Aerial part & W & 3 \\
\hline & $\begin{array}{l}\text { Ocimum basilicum L. } \\
\text { (BCN29897) }\end{array}$ & Alfàbrega & $\begin{array}{l}\text { High-grade alcoholic } \\
\text { beverage }\end{array}$ & Aerial part & C & 6 \\
\hline & $\begin{array}{l}\text { Origanum majorana } \\
\text { L. (BCN113585) }\end{array}$ & Marduix & $\begin{array}{l}\text { Condiment. High-grade } \\
\text { alcoholic beverage }\end{array}$ & Aerial part. Flower & C & 27 \\
\hline & $\begin{array}{l}\text { Origanum vulgare L. } \\
\text { (BCN113705) }\end{array}$ & Orenga & $\begin{array}{l}\text { Condiment. High-grade } \\
\text { alcoholic beverage. Not } \\
\text { reported }\end{array}$ & Aerial part & W & 18 \\
\hline & $\begin{array}{l}\text { Prunella grandiflora } \\
\text { (L.) Scholler } \\
\text { (BCN24956) }\end{array}$ & Herba del traïdor & $\begin{array}{l}\text { High-grade alcoholic } \\
\text { beverage }\end{array}$ & Flower & W & 1 \\
\hline & $\begin{array}{l}\text { Prunella laciniata (L.) } \\
\text { L. (BCN29481) }\end{array}$ & Herba del traïdor & $\begin{array}{l}\text { High-grade alcoholic } \\
\text { beverage }\end{array}$ & Flower & W & 1 \\
\hline & $\begin{array}{l}\text { Prunella vulgaris } \mathrm{L} \text {. } \\
\text { (BCN113578) }\end{array}$ & Herba del traïdor & $\begin{array}{l}\text { High-grade alcoholic } \\
\text { beverage }\end{array}$ & Flower & W & 2 \\
\hline & $\begin{array}{l}\text { Rosmarinus } \\
\text { officinalis L. } \\
\text { (BCN113599) }\end{array}$ & Romaní & $\begin{array}{l}\text { Condiment. High-grade } \\
\text { alcoholic beverage. Raw }\end{array}$ & Aerial part. Flower & W & 27 \\
\hline & $\begin{array}{l}\text { Salvia microphylla } \\
\text { Humb., Bonpl. \& } \\
\text { Kunth (BCN113718) }\end{array}$ & $\begin{array}{l}\text { Menta. Menta americana. Menta } \\
\text { romana }\end{array}$ & $\begin{array}{l}\text { High-grade alcoholic } \\
\text { beverage }\end{array}$ & Aerial part & C & 4 \\
\hline & $\begin{array}{l}\text { Salvia officinalis } \mathrm{L} \text {. } \\
\text { subsp. lavandulifolia }\end{array}$ & Sàlvia de fulla estreta & $\begin{array}{l}\text { High-grade alcoholic } \\
\text { beverage }\end{array}$ & Aerial part & W & 1 \\
\hline
\end{tabular}


Table 4 Food plants reported in the studied area (Continued)

\begin{tabular}{|c|c|c|c|c|c|c|}
\hline Family & Taxon (voucher) & Catalan vernacular names & Preparation & Used part & $\begin{array}{l}\text { Wild }(\mathrm{W}) / \\
\text { cultivated }(\mathrm{C})\end{array}$ & $\begin{array}{l}\text { Use } \\
\text { reports }\end{array}$ \\
\hline & $\begin{array}{l}\text { Salvia officinalis L. } \\
\text { subsp. officinalis } \\
\text { (BCN113583) }\end{array}$ & Sàlvia. Sàlvia de fulla ampla & $\begin{array}{l}\text { Condiment. High-grade } \\
\text { alcoholic beverage }\end{array}$ & $\begin{array}{l}\text { Aerial part. Flower. } \\
\text { Leaf }\end{array}$ & C & 11 \\
\hline & $\begin{array}{l}\text { Satureja montana L. } \\
\text { (BCN113741) }\end{array}$ & Sajolida & $\begin{array}{l}\text { Condiment. High-grade } \\
\text { alcoholic beverage }\end{array}$ & Aerial part & W & 12 \\
\hline & $\begin{array}{l}\text { Sideritis hirsuta L. } \\
\text { (BCN113582) }\end{array}$ & Herba de Sant Antoni & $\begin{array}{l}\text { High-grade alcoholic } \\
\text { beverage }\end{array}$ & Aerial part & W & 1 \\
\hline & $\begin{array}{l}\text { Stachys byzantina C. } \\
\text { Koch (BCN113707) }\end{array}$ & $\begin{array}{l}\text { Fulles de la mare de Déu. Planta de } \\
\text { vellut }\end{array}$ & $\begin{array}{l}\text { High-grade alcoholic } \\
\text { beverage }\end{array}$ & Leaf & C & 3 \\
\hline & $\begin{array}{l}\text { Stachys officinalis (L.) } \\
\text { Trevisan (BCN25011) }\end{array}$ & Brotònica & $\begin{array}{l}\text { High-grade alcoholic } \\
\text { beverage }\end{array}$ & Flower & C & 2 \\
\hline & $\begin{array}{l}\text { Teucrium } \\
\text { chamaedrys L. } \\
\text { (BCN29806) }\end{array}$ & Brotònica & $\begin{array}{l}\text { High-grade alcoholic } \\
\text { beverage }\end{array}$ & Aerial part & W & 1 \\
\hline & $\begin{array}{l}\text { Thymus xcitriodorus } \\
\text { (Pers.) Schreber } \\
\text { (BCN113803) }\end{array}$ & $\begin{array}{l}\text { Farigola de xocolata. Farigola } \\
\text { llimonera }\end{array}$ & $\begin{array}{l}\text { High-grade alcoholic } \\
\text { beverage }\end{array}$ & Aerial part & C & 1 \\
\hline & $\begin{array}{l}\text { Thymus serpyllum L. } \\
\text { (BCN113719) }\end{array}$ & $\begin{array}{l}\text { Farigola de pastor. Farigoleta. Salsa } \\
\text { de pastor }\end{array}$ & $\begin{array}{l}\text { Boiled in water. } \\
\text { Condiment. High-grade } \\
\text { alcoholic beverage }\end{array}$ & Aerial part & W & 11 \\
\hline & $\begin{array}{l}\text { Thymus vulgaris } \mathrm{L} \text {. } \\
\text { (BCN113590) }\end{array}$ & Farigola & $\begin{array}{l}\text { Condiment. High-grade } \\
\text { alcoholic beverage }\end{array}$ & Aerial part & W & 34 \\
\hline \multirow[t]{2}{*}{ Lauraceae } & $\begin{array}{l}\text { Cinnamomum } \\
\text { zeylanicum Nees } \\
\text { (BCN47283) }\end{array}$ & Canyella & $\begin{array}{l}\text { Condiment. High-grade } \\
\text { alcoholic beverage }\end{array}$ & Bark & $C$ & 10 \\
\hline & $\begin{array}{l}\text { Laurus nobilis L. } \\
\text { (BCN113717) }\end{array}$ & Llord. Llorer & $\begin{array}{l}\text { Condiment. High-grade } \\
\text { alcoholic beverage }\end{array}$ & Aerial part. Leaf & $C$ & 36 \\
\hline Liliaceae & $\begin{array}{l}\text { Lilium candidum } \mathrm{L} \text {. } \\
\text { (BCN46841) }\end{array}$ & $\begin{array}{l}\text { Lliri de Sant Antoni. Lliri de Sant } \\
\text { Josep }\end{array}$ & $\begin{array}{l}\text { High-grade alcoholic } \\
\text { beverage }\end{array}$ & Flower & C & 4 \\
\hline Lythraceae & $\begin{array}{l}\text { Punica granatum L. } \\
\text { (BCN29764) }\end{array}$ & $\begin{array}{l}\text { Magrana (fruit). Magraner. Magraner } \\
\text { agre. Magraner bord. Magraner dolç }\end{array}$ & $\begin{array}{l}\text { Cooked with sugar. High- } \\
\text { grade alcoholic beverage. } \\
\text { Raw }\end{array}$ & Flower. Fruit & C & 6 \\
\hline Magnoliaceae & $\begin{array}{l}\text { Magnolia grandiflora } \\
\text { L. (BCN64396) }\end{array}$ & Magnòlia & $\begin{array}{l}\text { High-grade alcoholic } \\
\text { beverage }\end{array}$ & Flower & C & 2 \\
\hline \multirow[t]{5}{*}{ Malvaceae } & $\begin{array}{l}\text { Althaea officinalis L. } \\
\text { (BCN113799) }\end{array}$ & Malví & $\begin{array}{l}\text { High-grade alcoholic } \\
\text { beverage }\end{array}$ & Root & W & 1 \\
\hline & $\begin{array}{l}\text { Malva sylvestris L. } \\
\text { (BCN29889) }\end{array}$ & Malva. Malva rosa & $\begin{array}{l}\text { High-grade alcoholic } \\
\text { beverage }\end{array}$ & Flower & W & 8 \\
\hline & $\begin{array}{l}\text { Theobroma cacao L. } \\
\text { (BCN30763) }\end{array}$ & Xocolata (elaborated product) & Condiment & Seed & $C$ & 1 \\
\hline & $\begin{array}{l}\text { Tilia cordata Mill. } \\
\text { (BCN26784) }\end{array}$ & Tillla & $\begin{array}{l}\text { High-grade alcoholic } \\
\text { beverage }\end{array}$ & $\begin{array}{l}\text { Bract with } \\
\text { inflorescence }\end{array}$ & W & 5 \\
\hline & $\begin{array}{l}\text { Tilia platyphyllos } \\
\text { Scop. (BCN113739) }\end{array}$ & Tei. Til.la. Til.ler de bosc & $\begin{array}{l}\text { High-grade alcoholic } \\
\text { beverage }\end{array}$ & $\begin{array}{l}\text { Bract with } \\
\text { inflorescence }\end{array}$ & W & 7 \\
\hline Moraceae & $\begin{array}{l}\text { Ficus carica L. } \\
\text { (BCN24887) }\end{array}$ & $\begin{array}{l}\text { Figa (infructescence). Figa d'Alacant } \\
\text { (infructescence). Figa de coll de } \\
\text { senyora (infructescence). Figa de coll } \\
\text { llarg blanca (infructescence). Figa de } \\
\text { coll llarg negra (infructescence). Figa } \\
\text { de pota de cavall (infructescence). } \\
\text { Figa de Sant Joan (infructescence). } \\
\text { Figa negra (infructescence). Figuera. } \\
\text { Figuera de coll de senyora }\end{array}$ & $\begin{array}{l}\text { Air dried. Cooked with } \\
\text { sugar. Raw }\end{array}$ & Infructescence & $C$ & 11 \\
\hline Myristicaceae & $\begin{array}{l}\text { Myristica fragrans } \\
\text { Houtt. (BCN50769) }\end{array}$ & Nou moscada & $\begin{array}{l}\text { High-grade alcoholic } \\
\text { beverage }\end{array}$ & Fruit & $C$ & 6 \\
\hline Myrtaceae & Eucalyptus globulus & Eucaliptu. Eucaliptus & High-grade alcoholic & Leaf & $C$ & 2 \\
\hline
\end{tabular}


Table 4 Food plants reported in the studied area (Continued)

\begin{tabular}{|c|c|c|c|c|c|c|}
\hline Family & Taxon (voucher) & Catalan vernacular names & Preparation & Used part & $\begin{array}{l}\text { Wild (W)/ } \\
\text { cultivated (C) }\end{array}$ & $\begin{array}{l}\text { Use } \\
\text { reports }\end{array}$ \\
\hline & Labill. (BCN29696) & & beverage & & & \\
\hline & $\begin{array}{l}\text { Syzygium } \\
\text { aromaticum (L.) } \\
\text { Merr. et Perry } \\
\text { (BCN47279) }\end{array}$ & $\begin{array}{l}\text { Clau d'espècia. Clau de pot. Clavell. } \\
\text { Clavell d'espècia }\end{array}$ & $\begin{array}{l}\text { High-grade alcoholic } \\
\text { beverage }\end{array}$ & Floral bud & C & 7 \\
\hline \multirow[t]{3}{*}{ Oleaceae } & $\begin{array}{l}\text { Ligustrum vulgare L. } \\
\text { (BCN24915) }\end{array}$ & Olivereta & $\begin{array}{l}\text { High-grade alcoholic } \\
\text { beverage }\end{array}$ & Flower & W & 2 \\
\hline & $\begin{array}{l}\text { Olea europaea L. } \\
\text { subsp. europaea } \\
\text { (BCN29898) }\end{array}$ & $\begin{array}{l}\text { Oli (elaborated product). Oli d'oliva } \\
\text { (elaborated product). Olivera. Oliva } \\
\text { (fruit) }\end{array}$ & $\begin{array}{l}\text { Condiment. Boiled in } \\
\text { water. High-grade alco- } \\
\text { holic beverage. Preserved } \\
\text { in salt and water. Raw }\end{array}$ & $\begin{array}{l}\text { Aerial part. Flower. } \\
\text { Fruit }\end{array}$ & $C$ & 22 \\
\hline & $\begin{array}{l}\text { Syringa vulgaris L. } \\
\text { (BCN29959) }\end{array}$ & Lilà & $\begin{array}{l}\text { High-grade alcoholic } \\
\text { beverage }\end{array}$ & Flower & C & 2 \\
\hline \multirow[t]{2}{*}{ Papaveraceae } & $\begin{array}{l}\text { Papaver rhoeas L. } \\
\text { (BCN29903) }\end{array}$ & Gallaret. Pipiripip. Quiquiriquí. Rosella & $\begin{array}{l}\text { Boiled in water. High- } \\
\text { grade alcoholic beverage. } \\
\text { Raw }\end{array}$ & Aerial part. Leaf & W & 4 \\
\hline & $\begin{array}{l}\text { Papaver somniferum } \\
\text { L. (BCN24941) }\end{array}$ & Cascall & $\begin{array}{l}\text { High-grade alcoholic } \\
\text { beverage }\end{array}$ & Aerial part & W & 2 \\
\hline \multirow[t]{3}{*}{ Pinaceae } & $\begin{array}{l}\text { Pinus halepensis Mill. } \\
\text { (BCN113592) }\end{array}$ & $\begin{array}{l}\text { Pi. Pi blanc. Pi bord. Pi de pinya } \\
\text { llarga. Pi petit. Pinya (fructification) }\end{array}$ & $\begin{array}{l}\text { High-grade alcoholic } \\
\text { beverage }\end{array}$ & $\begin{array}{l}\text { Flower. } \\
\text { Fructification. } \\
\text { Young shoot }\end{array}$ & W & 10 \\
\hline & $\begin{array}{l}\text { Pinus pinaster Ait. } \\
\text { (BCN36559) }\end{array}$ & Pi bord. Pi melis & $\begin{array}{l}\text { Air dried. High-grade al- } \\
\text { coholic beverage }\end{array}$ & Flower. Leaf & W & 2 \\
\hline & $\begin{array}{l}\text { Pinus pinea } \mathrm{L} \text {. } \\
\text { (BCN26751) }\end{array}$ & Pi. Pi de llei. Pi de pinya. Pi pinyer & $\begin{array}{l}\text { High-grade alcoholic } \\
\text { beverage }\end{array}$ & Fructification & W & 1 \\
\hline \multirow[t]{2}{*}{ Plantaginaceae } & $\begin{array}{l}\text { Plantago lanceolata } \\
\text { L. (BCN32138) }\end{array}$ & $\begin{array}{l}\text { Plantatge de fulla estreta. Plantatge } \\
\text { estret }\end{array}$ & $\begin{array}{l}\text { High-grade alcoholic } \\
\text { beverage }\end{array}$ & Flower. Leaf & W & 4 \\
\hline & $\begin{array}{l}\text { Plantago major L. } \\
\text { (BCN29910) }\end{array}$ & $\begin{array}{l}\text { Plantatge. Plantatge ample. Plantatge } \\
\text { de fulla ampla }\end{array}$ & $\begin{array}{l}\text { High-grade alcoholic } \\
\text { beverage }\end{array}$ & Flower. Leaf & W & 5 \\
\hline \multirow[t]{10}{*}{ Poaceae } & $\begin{array}{l}\text { Avena barbata Pott } \\
\text { ex Link in Schrad. } \\
\text { (BCN49867) }\end{array}$ & Avena. Cugula & $\begin{array}{l}\text { High-grade alcoholic } \\
\text { beverage }\end{array}$ & Fruit & W & 2 \\
\hline & $\begin{array}{l}\text { Avena sativa L. } \\
\text { (BCN29839) }\end{array}$ & Civada & $\begin{array}{l}\text { High-grade alcoholic } \\
\text { beverage }\end{array}$ & Fruit & C & 2 \\
\hline & $\begin{array}{l}\text { Briza media L. } \\
\text { (BCN113733) }\end{array}$ & Belluguets & $\begin{array}{l}\text { High-grade alcoholic } \\
\text { beverage }\end{array}$ & Flower & W & 2 \\
\hline & $\begin{array}{l}\text { Hordeum vulgare L. } \\
\text { (BCN46843) }\end{array}$ & Ordi & Cooked & Fruit & C & 1 \\
\hline & $\begin{array}{l}\text { Panicum miliaceum } \\
\text { L. (BCN12911) }\end{array}$ & Mill & Air dried & Fruit & C & 2 \\
\hline & $\begin{array}{l}\text { Saccharum } \\
\text { officinarum L. } \\
\text { (BCN50771) }\end{array}$ & $\begin{array}{l}\text { Rom (elaborated product). Sucre roig } \\
\text { (elaborated product) }\end{array}$ & $\begin{array}{l}\text { Alcoholic beverage made } \\
\text { with wine. High-grade al- } \\
\text { coholic beverage }\end{array}$ & Stem & C & 4 \\
\hline & $\begin{array}{l}\text { Secale cereale } \mathrm{L} . \\
\text { (BCN46828) }\end{array}$ & Sègol. Sègal & Boiled in water. Cooked & Fruit & C & 7 \\
\hline & $\begin{array}{l}\text { Sorghum bicolor (L.) } \\
\text { Moench } \\
\text { (BCN31310) }\end{array}$ & Melca. Sorgo & Cooked & Fruit & $C$ & 1 \\
\hline & $\begin{array}{l}\text { Triticum aestivum L. } \\
\text { (BCN29963) }\end{array}$ & $\begin{array}{l}\text { Blat. Farina (elaborated product). Pa } \\
\text { (elaborated product). Palla } \\
\text { (elaborated product). Segó (bran) }\end{array}$ & $\begin{array}{l}\text { Air dried. Boiled in water. } \\
\text { Cooked. High-grade alco- } \\
\text { holic beverage }\end{array}$ & Bran. Fruit. Spike & $C$ & 21 \\
\hline & $\begin{array}{l}\text { Zea mays L. } \\
\text { (BCN29830) }\end{array}$ & $\begin{array}{l}\text { Blat de morassa. Blat de moret. Blat } \\
\text { de moro. Farro (elaborated product) }\end{array}$ & $\begin{array}{l}\text { Air dried. Boiled in water. } \\
\text { Cooked. High-grade alco- } \\
\text { holic beverage }\end{array}$ & $\begin{array}{l}\text { Fruit. Styles and } \\
\text { stigmas }\end{array}$ & $C$ & 26 \\
\hline Polygonaceae & Fagopyrum & Fajol & Boiled in water. Cooked & Seed & C & 16 \\
\hline
\end{tabular}


Table 4 Food plants reported in the studied area (Continued)

\begin{tabular}{|c|c|c|c|c|c|c|}
\hline Family & Taxon (voucher) & Catalan vernacular names & Preparation & Used part & $\begin{array}{l}\text { Wild (W)/ } \\
\text { cultivated (C) }\end{array}$ & $\begin{array}{l}\text { Use } \\
\text { reports }\end{array}$ \\
\hline & $\begin{array}{l}\text { esculentum Moench } \\
\text { (BCN24886) }\end{array}$ & & & & & \\
\hline Portulacaceae & $\begin{array}{l}\text { Portulaca oleracea L. } \\
\text { (BCN46835) }\end{array}$ & Verdolaga & $\begin{array}{l}\text { Boiled in water. High- } \\
\text { grade alcoholic beverage. } \\
\text { Raw }\end{array}$ & Aerial part & W & 23 \\
\hline \multirow[t]{2}{*}{ Ranunculaceae } & $\begin{array}{l}\text { Anemone hepatica } \\
\text { L. (BCN29834) }\end{array}$ & Herba fetgera & $\begin{array}{l}\text { High-grade alcoholic } \\
\text { beverage }\end{array}$ & Aerial part. Leaf & W & 5 \\
\hline & $\begin{array}{l}\text { Clematis recta L. } \\
\text { (BCN113720) }\end{array}$ & Viadella & $\begin{array}{l}\text { High-grade alcoholic } \\
\text { beverage }\end{array}$ & Flower & W & 1 \\
\hline Rhamnaceae & $\begin{array}{l}\text { Ziziphus jujuba Mill. } \\
\text { (BCN113700) }\end{array}$ & Gínjol (fruit). Ginjoler & Raw & Fruit & C & 3 \\
\hline \multirow[t]{13}{*}{ Rosaceae } & $\begin{array}{l}\text { Agrimonia eupatoria } \\
\text { L. (BCN-E-193) }\end{array}$ & Herba cuquera & $\begin{array}{l}\text { High-grade alcoholic } \\
\text { beverage }\end{array}$ & Flower & W & 2 \\
\hline & $\begin{array}{l}\text { Crataegus } \\
\text { monogyna Jacq. } \\
\text { (BCN29858) }\end{array}$ & Arç. Arç blanc & $\begin{array}{l}\text { Cooked in oil. High-grade } \\
\text { alcoholic beverage }\end{array}$ & Flower & W & 4 \\
\hline & $\begin{array}{l}\text { Cydonia oblonga } \\
\text { Mill. (BCN46849) }\end{array}$ & $\begin{array}{l}\text { Codony (fruit). Codonyat (elaborated } \\
\text { product). Codonyer }\end{array}$ & $\begin{array}{l}\text { Cooked with sugar. High- } \\
\text { grade alcoholic beverage }\end{array}$ & Fruit & C & 24 \\
\hline & $\begin{array}{l}\text { Fragaria vesca L. } \\
\text { (BCN29697) }\end{array}$ & $\begin{array}{l}\text { Maduixa (infructescence). Maduixa } \\
\text { de bosc (infructescence). Maduixa } \\
\text { petita (infructescence). Maduixer. } \\
\text { Maduixer de bosc. Maduixeta } \\
\text { (infructescence) }\end{array}$ & Raw & Infructescence & W & 16 \\
\hline & $\begin{array}{l}\text { Fragaria viridis } \\
\text { Weston (BCN62767) }\end{array}$ & Marrans & Raw & Infructescence & C & 1 \\
\hline & $\begin{array}{l}\text { Mespilus germanica } \\
\text { L. (BCN50768) }\end{array}$ & $\begin{array}{l}\text { Nespler. Nespla de bosc. Nespra. } \\
\text { Nespro }\end{array}$ & Raw & Fruit & C & 9 \\
\hline & $\begin{array}{l}\text { Prunus avium (L.) L. } \\
\text { (BCN29827) }\end{array}$ & Cirera (fruit). Cirerer & $\begin{array}{l}\text { High-grade alcoholic } \\
\text { beverage. Raw }\end{array}$ & Flower. Fruit & C & 6 \\
\hline & $\begin{array}{l}\text { Prunus domestica L. } \\
\text { subsp. domestica } \\
\text { (BCN46834) }\end{array}$ & $\begin{array}{l}\text { Pruna (fruit). Pruner. Pruna clàudia } \\
\text { (fruit). Pruna de colló de frare (fruit) }\end{array}$ & $\begin{array}{l}\text { Cooked with sugar. High- } \\
\text { grade alcoholic beverage. } \\
\text { Raw }\end{array}$ & Flower. Fruit & C & 10 \\
\hline & $\begin{array}{l}\text { Prunus dulcis (Mill.) } \\
\text { Weeb. (BCN46833) }\end{array}$ & Ametller & $\begin{array}{l}\text { High-grade alcoholic } \\
\text { beverage }\end{array}$ & Flower & C & 1 \\
\hline & $\begin{array}{l}\text { Prunus persica (L.) } \\
\text { Batsch (BCN46832) }\end{array}$ & $\begin{array}{l}\text { Préssec (fruit). Préssec cardinal (fruit). } \\
\text { Préssec de coure (fruit). Préssec } \\
\text { duran (fruit). Préssec groc (fruit). } \\
\text { Préssec groc d'agost (fruit). Préssec } \\
\text { mollar (fruit). Préssec sang de llebre } \\
\text { (fruit). Préssec de Sant Joan (fruit). } \\
\text { Préssec de Sant Pere (fruit). } \\
\text { Presseguer }\end{array}$ & $\begin{array}{l}\text { Cooked with sugar. High- } \\
\text { grade alcoholic beverage. } \\
\text { Raw }\end{array}$ & Flower. Fruit & C & 9 \\
\hline & $\begin{array}{l}\text { Prunus spinosa L. } \\
\text { (BCN30005) }\end{array}$ & $\begin{array}{l}\text { Aranyó (fruit). Aranyoner. Arç. Arç } \\
\text { negre. Arça }\end{array}$ & $\begin{array}{l}\text { Cooked with sugar. High- } \\
\text { grade alcoholic beverage. } \\
\text { Raw }\end{array}$ & Fruit & W & 13 \\
\hline & $\begin{array}{l}\text { Pyrus communis L. } \\
\text { subsp. communis } \\
\text { (BCN46831) }\end{array}$ & $\begin{array}{l}\text { Pera (fruit). Pera conference (fruit). } \\
\text { Pera de Sant Joan (fruit). Pera rogija } \\
\text { (fruit). Perer. Perer mau }\end{array}$ & $\begin{array}{l}\text { Alcoholic beverage made } \\
\text { with wine. Cooked with } \\
\text { sugar. High-grade alco- } \\
\text { holic beverage. Raw }\end{array}$ & Flower. Fruit & C & 8 \\
\hline & $\begin{array}{l}\text { Pyrus malus L. } \\
\text { subsp. mitis (Wallr.) } \\
\text { O.Bolòs et J.Vigo } \\
\text { (BCN46830) }\end{array}$ & $\begin{array}{l}\text { Poma (fruit). Poma aspra (fruit). Poma } \\
\text { cambusina (fruit). Poma camosa } \\
\text { (fruit). Poma capçana (fruit). Poma } \\
\text { del ciri (fruit). Poma del ciri groga } \\
\text { (fruit). Poma del ciri vermella (fruit). } \\
\text { Poma golden (fruit). Poma rodona } \\
\text { (fruit). Poma royal (fruit). Pomer. } \\
\text { Pomer del ciri. Pomera. Pomera del } \\
\text { ciri }\end{array}$ & $\begin{array}{l}\text { Cooked with sugar. High- } \\
\text { grade alcoholic beverage. } \\
\text { Raw }\end{array}$ & Flower. Fruit & C & 24 \\
\hline
\end{tabular}


Table 4 Food plants reported in the studied area (Continued)

\begin{tabular}{|c|c|c|c|c|c|c|}
\hline Family & Taxon (voucher) & Catalan vernacular names & Preparation & Used part & $\begin{array}{l}\text { Wild (W)/ } \\
\text { cultivated (C) }\end{array}$ & $\begin{array}{l}\text { Use } \\
\text { reports }\end{array}$ \\
\hline & $\begin{array}{l}\text { Rosa canina L. } \\
\text { (BCN29772) }\end{array}$ & Rosa. Rosa de pastor. Roser & $\begin{array}{l}\text { High-grade alcoholic } \\
\text { beverage }\end{array}$ & Flower & W & 6 \\
\hline & Rosa sp. & Rosa. Rosa de jardí. Roser & $\begin{array}{l}\text { High-grade alcoholic } \\
\text { beverage }\end{array}$ & Flower. Leaf & C & 2 \\
\hline & $\begin{array}{l}\text { Rubus idaeus L. } \\
\text { (BCN29774) }\end{array}$ & Gerd (fruit) & Cooked with sugar. Raw & Fruit & W & 3 \\
\hline & $\begin{array}{l}\text { Rubus ulmifolius } \\
\text { Schott (BCN29938) }\end{array}$ & $\begin{array}{l}\text { Bardissa. Mora (fruit). Mora negra } \\
\text { (fruit). Romeguera }\end{array}$ & $\begin{array}{l}\text { Cooked with sugar. High- } \\
\text { grade alcoholic beverage. } \\
\text { Raw }\end{array}$ & $\begin{array}{l}\text { Flower. Fruit. } \\
\text { Young shoot }\end{array}$ & W & 34 \\
\hline & $\begin{array}{l}\text { Sorbus domestica L. } \\
\text { (BCN46827) }\end{array}$ & Server & Raw & Fruit & C & 1 \\
\hline & $\begin{array}{l}\text { Sorbus torminalis (L.) } \\
\text { Crantz (BCN43294) }\end{array}$ & - & Raw & Fruit & W & 1 \\
\hline \multirow[t]{2}{*}{ Rubiaceae } & $\begin{array}{l}\text { Asperula cynanchica } \\
\text { L. (BCN29634) }\end{array}$ & Herbaprima & $\begin{array}{l}\text { High-grade alcoholic } \\
\text { beverage }\end{array}$ & Aerial part & w & 1 \\
\hline & $\begin{array}{l}\text { Coffea arabica L. } \\
(\mathrm{BCN} 46852)^{\mathrm{a}}\end{array}$ & Cafè & $\begin{array}{l}\text { Beverage made with } \\
\text { water. High-grade alco- } \\
\text { holic beverage }\end{array}$ & Seed & C & 9 \\
\hline \multirow[t]{5}{*}{ Rutaceae } & $\begin{array}{l}\text { Citrus aurantium L. } \\
\text { (BCN46080) }\end{array}$ & $\begin{array}{l}\text { Taronger agre. Taronger amarg. } \\
\text { Taronger bord. Taronja agra (fruit) }\end{array}$ & $\begin{array}{l}\text { Condiment. Cooked with } \\
\text { sugar. High-grade alco- } \\
\text { holic beverage. }\end{array}$ & Leaf. Fruit & C & 4 \\
\hline & $\begin{array}{l}\text { Citrus japonica } \\
\text { Thunb. (BCN113966) }\end{array}$ & Llimona de Xipre & $\begin{array}{l}\text { High-grade alcoholic } \\
\text { beverage }\end{array}$ & Fruit & C & 1 \\
\hline & $\begin{array}{l}\text { Citrus limon }(L .) \\
\text { Burm. (BCN46853) }\end{array}$ & Llimona (fruit). Llimoner & $\begin{array}{l}\text { Condiment. High-grade } \\
\text { alcoholic beverage. Raw }\end{array}$ & Epicarp. Fruit. Leaf & C & 19 \\
\hline & $\begin{array}{l}\text { Citrus sinensis (L.) } \\
\text { Osbeck (BCN24752) }\end{array}$ & $\begin{array}{l}\text { Taronger. Taronger dolç. Taronja } \\
\text { (fruit) }\end{array}$ & $\begin{array}{l}\text { Condiment. Cooked with } \\
\text { sugar. High-grade alco- } \\
\text { holic beverage }\end{array}$ & $\begin{array}{l}\text { Epicarp. Flower. } \\
\text { Fruit }\end{array}$ & C & 15 \\
\hline & $\begin{array}{l}\text { Ruta chalepensis L. } \\
\text { (BCN29940) }\end{array}$ & Ruda & $\begin{array}{l}\text { Condiment. High-grade } \\
\text { alcoholic beverage. Raw }\end{array}$ & Aerial part. Leaf & W & 17 \\
\hline Schisandraceae & $\begin{array}{l}\text { Illicium verum } \\
\text { Hook.f. (BCN47282) }\end{array}$ & Anís estrellat & $\begin{array}{l}\text { High-grade alcoholic } \\
\text { beverage }\end{array}$ & Fruit & C & 4 \\
\hline Scrophulariaceae & Verbascum sp. & Cua de guilla & $\begin{array}{l}\text { High-grade alcoholic } \\
\text { beverage }\end{array}$ & Flower & W & 1 \\
\hline \multirow[t]{3}{*}{ Solanaceae } & $\begin{array}{l}\text { Capsicum annuum } \\
\text { L. (BCN42737) }\end{array}$ & Bitxo & $\begin{array}{l}\text { Cooked in oil. Preserved } \\
\text { in salt and water }\end{array}$ & Fruit & C & 12 \\
\hline & $\begin{array}{l}\text { Solanum } \\
\text { lycopersicum L. } \\
\text { (BCN29952) }\end{array}$ & $\begin{array}{l}\text { Tomata (fruit). Tomata de guardar } \\
\text { (fruit). Tomata de la meta (fruit). } \\
\text { Tomata de penjar (fruit). Tomata dels } \\
\text { tres cantos (fruit). Tomata plena } \\
\text { (fruit). Tomata poma (fruit) }\end{array}$ & $\begin{array}{l}\text { Cooked in oil. Preserved } \\
\text { in salt and water. Cooked } \\
\text { with sugar. Raw }\end{array}$ & Fruit & C & 12 \\
\hline & $\begin{array}{l}\text { Solanum tuberosum } \\
\text { L. (BCN29797) }\end{array}$ & Patata. Patatera. Trumfera & Boiled in water. Cooked & Tuber & C & 3 \\
\hline Ulmaceae & $\begin{array}{l}\text { Ulmus minor Mill. } \\
\text { (BCN113729) }\end{array}$ & Om & Boiled in water & Leaf & W & 1 \\
\hline \multirow[t]{2}{*}{ Urticaceae } & $\begin{array}{l}\text { Parietaria officinalis } \\
\text { L. subsp. judaica (L.) } \\
\text { Béguinot } \\
\text { (BCN113715) }\end{array}$ & Blet de paret. Mollerosa & $\begin{array}{l}\text { High-grade alcoholic } \\
\text { beverage }\end{array}$ & Aerial part & W & 5 \\
\hline & $\begin{array}{l}\text { Urtica dioica L. } \\
\text { (BCN29814) }\end{array}$ & Ortiga & Boiled in water & Aerial part & W & 4 \\
\hline Verbenaceae & $\begin{array}{l}\text { Lippia triphylla } \\
\text { (L'Hér.) O.Kuntze } \\
\text { (BCN29886) }\end{array}$ & Marialluïsa & $\begin{array}{l}\text { High-grade alcoholic } \\
\text { beverage }\end{array}$ & Aerial part. Leaf & C & 12 \\
\hline
\end{tabular}


Table 4 Food plants reported in the studied area (Continued)

\begin{tabular}{|c|c|c|c|c|c|c|}
\hline Family & Taxon (voucher) & Catalan vernacular names & Preparation & Used part & $\begin{array}{l}\text { Wild }(\mathrm{W}) / \\
\text { cultivated }(\mathrm{C})\end{array}$ & $\begin{array}{l}\text { Use } \\
\text { reports }\end{array}$ \\
\hline Violaceae & $\begin{array}{l}\text { Viola tricolor } \mathrm{L} . \\
\text { (BCN25041) }\end{array}$ & Pensaments & $\begin{array}{l}\text { High-grade alcoholic } \\
\text { beverage }\end{array}$ & Flower & W & 2 \\
\hline Vitaceae & $\begin{array}{l}\text { Vitis vinifera L. } \\
\text { (BCN29972) }\end{array}$ & $\begin{array}{l}\text { Raïm (fruit). Sarment. Vi (elaborated } \\
\text { product). Vinagre (elaborated } \\
\text { product). Vinya }\end{array}$ & $\begin{array}{l}\text { Condiment. Cooked with } \\
\text { sugar. Preserved in } \\
\text { vinegar. Raw }\end{array}$ & Fruit & C & 12 \\
\hline
\end{tabular}

${ }^{\mathrm{a}}$ In our country, most coffee industrial presentations are based on C. arabica, the other taxa, such as C. canephora Pierre ex A.Froehner and C. liberica Hiern being clearly minority

34], the so-called brunyols or bunyols. These are kind of pastry prepared coating the flowers with a pasta made with floor and water, frying them in very hot oil, and finally seasoning them with salt or sugar, depending on the use of the product with salty food or as a dessert. Additionally, and more originally, the flowers (optionally together with tender leaves) of this species are prepared and consumed in omelet.

For human food, the use of cultivated and wild plants is similar in percentages: $44.62 \%$ of the taxa used are cultivated and $55.38 \%$ are wild. Contrarily to what one could expect, with crops dominating the market, the relevance of wild food plants is high.

Concerning animal fodder (Table 5), the most reported families are Poaceae (153 UR), Fabaceae (105 UR), and Brassicaceae (70 UR) the five more cited species being Zea mays (56 UR), Brassica napus (46 UR), Quercus ilex (37 UR), Medicago sativa (34 UR), and Triticum aestivum (28 UR). In general, these species are consumed raw $(43.88 \%)$ or air-dried and preserved $(39.12 \%)$. The most used parts of plants are the aerial part that sometimes includes the whole plant (41.68\%), leaves (19.74\%), and seeds (14.1\%). These grains can be given directly to the animals or processed in order to obtain flour or fodder.

For animal feed, the percentage repartition is similar than for human food: $44.44 \%$ of taxa used are cultivated and $55.56 \%$ are wild, again accounting for the importance of food plants in the region considered.

\section{Other uses}

This category, arranged in Table 6, includes uses that are neither medicinal or food. This is a melting pot with numerous subcategories. Most probably, in societies currently much more dependent on natural resources at an ethnobotanical level, many subcategories could be treated independently, because they would receive a big number of use reports, but we have realized that in our cultural area, where many uses have only few reports based on ancient memories, it is practical to treat all of them together (Gras et al. 2016). We have collected 894 UR concerning 125 taxa, 8 of them only determined at the genus level. These taxa belong to 47 plant families,
Fagaceae (136 UR, 15.21\%), Poaceae (117 UR; 13.09\%), Ericaceae (109 UR; 12.19\%), Cannabaceae (69 UR; $7.72 \%$ ), and Fabaceae (48 UR; 5.37\%) being the most cited.

The five most reported species are Quercus ilex (66 UR; 7.38\%), Celtis australis (63 UR; 7.05\%), Arundo donax (48 UR; 5.37\%), Erica scoparia (44 UR; 4.92\%), and Laurus nobilis (39 UR; 4.36\%). This top list reflects the persistency of a rural bottom still alive in the studied territory, since these plants are importantly used for agricultural practices, e.g., Arundo to grow Lycopersicon esculentum or Phaseolus vulgaris plants, Celtis to elaborate forks and Erica (as its specific epithet claims, indicating an old use) to make brooms (Fig. 2).

Even if we treat all of them in a single category, thus comparable with the medicinal and food ones, the different uses (subcategories) are also addressed, and those regarding the present study can be observed in Fig. 4. We emphasize the importance of the artisanal uses (231 UR, 25.84\%) comprising the making of shoes, toys, and brooms among others, agrosilvopastoral management (170 UR, 19.02\%) and timber (127 UR, 14.21\%). Some of these categories correspond to professions that no longer exist but that have had a lot of relevance in the past and are, in some cases, transformed to sell their products as touristic objects.

Regarding the parts of plant used, which are closely related with their uses, the most reported are the stem and trunk (364 UR; $40.72 \%$ ), followed by the aerial part and the whole plant (253 UR; $28.3 \%$ ), and the fruit and infructescence (65 UR; 8.50\%).

\section{Vernacular names}

In this study, 581 vernacular names for 306 species, subspecies, and varieties have been collected, comprising 2892 reports. Ten taxa have been mentioned by the informants without any popular name. This is a very small percentage of the phytonyms recorded, and reflects that, in a few cases, the informants do not know (or, more often, do not remember) the name of a plant. The most reported taxa are those with a single or a few vernacular names as a general trend, as is the case of thyme (Thymus vulgaris), 
Table 5 Fodder plants reported in the studied area

\begin{tabular}{|c|c|c|c|c|c|c|}
\hline Family & Taxon (voucher) & Catalan vernacular names & Preparation & Used part & $\begin{array}{l}\text { Wild }(\text { W)/ } \\
\text { cultivated } \\
\text { (C) }\end{array}$ & $\begin{array}{l}\text { Use } \\
\text { reports }\end{array}$ \\
\hline \multirow[t]{2}{*}{ Amaranthaceae } & $\begin{array}{l}\text { Beta vulgaris L. subsp. } \\
\text { vulgaris var. crassa } \\
\text { (Alef.) Helm } \\
\text { (BCN50761) }\end{array}$ & $\begin{array}{l}\text { Bleda. Polpa (elaborated product). Remolatxa. Sucre } \\
\text { (elaborated product). Sucre candi (elaborated } \\
\text { product) }\end{array}$ & $\begin{array}{l}\text { Air dried }{ }^{\mathrm{a}} \text {. } \\
\text { Boiled in } \text { water }^{\mathrm{a},} \\
\text { e. Raw }{ }^{\mathrm{b}, \mathrm{e}}\end{array}$ & $\begin{array}{l}\text { Root. Whole } \\
\text { plant }\end{array}$ & C & 25 \\
\hline & $\begin{array}{l}\text { Beta vulgaris L. subsp. } \\
\text { vulgaris var. vulgaris } \\
\text { (BCN46075) }\end{array}$ & Bleda & Boiled in water ${ }^{\mathrm{e}}$ & Aerial part & C & 3 \\
\hline Apiaceae & $\begin{array}{l}\text { Foeniculum vulgare } \\
\text { Mill. (BCN26350) }\end{array}$ & Fonoll & $\operatorname{Raw}^{f}$ & Aerial part & W & 4 \\
\hline Aquifoliaceae & $\begin{array}{l}\text { Ilex aquifolium L. } \\
\text { (BCN29876) }\end{array}$ & Grèvol & $\operatorname{Raw}^{f}$ & Leaf & W & 1 \\
\hline Araceae & $\begin{array}{l}\text { Arum italicum Mill. } \\
\text { (BCN32358) }\end{array}$ & Xàrria. Xèrria & $\begin{array}{l}\text { Boiled in } \\
\text { water }{ }^{\mathrm{e}} \text {. Raw }\end{array}$ & $\begin{array}{l}\text { Root. Whole } \\
\text { plant }\end{array}$ & W & 4 \\
\hline Araliaceae & $\begin{array}{l}\text { Hedera helix L. } \\
\text { (BCN29869) }\end{array}$ & Heura. Heura d'alzina & $\operatorname{Raw}^{a, g}$ & Leaf & W & 3 \\
\hline \multirow[t]{2}{*}{ Asparagaceae } & $\begin{array}{l}\text { Agave americana L. } \\
\text { (BCN46860) }\end{array}$ & Figuerassa & $\mathrm{e}_{\mathrm{B}}^{\text {Boiled in water }} \mathrm{a}^{\mathrm{a},}$ & Leaf & W & 5 \\
\hline & $\begin{array}{l}\text { Aphyllanthes } \\
\text { monspeliensis L. } \\
\text { (BCN29627) }\end{array}$ & Llonsa. Pa de cucut & $\operatorname{Raw}^{a}$ & Aerial part & W & 3 \\
\hline \multirow[t]{10}{*}{ Asteraceae } & $\begin{array}{l}\text { Centaurea jacea L. } \\
\text { (BCN21907) }\end{array}$ & Caps de burro & $\operatorname{Raw}^{f}$ & Aerial part & W & 1 \\
\hline & $\begin{array}{l}\text { Chondrilla juncea L. } \\
\text { (BCN29852) }\end{array}$ & Màstec & $\operatorname{Raw}^{f}$ & Aerial part & W & 2 \\
\hline & $\begin{array}{l}\text { Cichorium endivia } \mathrm{L} \text {. } \\
\text { (BCN46854) }\end{array}$ & Escarola & Raw & Leaf & C & 1 \\
\hline & $\begin{array}{l}\text { Cichorium intybus } \mathrm{L} . \\
\text { (BCN29660) }\end{array}$ & Cama-roja. Xicoia. Xicoina. Xicòria & $\operatorname{Raw}^{f}$ & Aerial part & W & 2 \\
\hline & $\begin{array}{l}\text { Reichardia picroides (L.) } \\
\text { Roth (BCN113704) }\end{array}$ & Cosconilla & $\operatorname{Raw}^{f}$ & Leaf & W & 6 \\
\hline & $\begin{array}{l}\text { Silybum marianum (L.) } \\
\text { Gaertn. (BCN29958) }\end{array}$ & Card & $\operatorname{Raw}^{\mathrm{b}}$ & Aerial part & W & 1 \\
\hline & $\begin{array}{l}\text { Sonchus oleraceus L. } \\
\text { (BCN113723) }\end{array}$ & Lletissó. Llipsó. Llistó & $\underset{g}{\text { Air dried }}{ }^{f}$. Raw ${ }^{f,}$ & Aerial part & W & 5 \\
\hline & Sonchus sp. & Llepsó. Lletissó. Llistó & $\operatorname{Raw}^{a, c, f}$ & Aerial part & W & 5 \\
\hline & $\begin{array}{l}\text { Sonchus tenerrimus L. } \\
\text { (BCN29954) }\end{array}$ & Lletissó. Llitsó & $\operatorname{Raw}^{f}$ & Aerial part & W & 3 \\
\hline & $\begin{array}{l}\text { Taraxacum officinale } \\
\text { Weber in Wiggers } \\
\text { (BCN25948) }\end{array}$ & Dent de lleó. Xicoia & $\operatorname{Raw}^{f}$ & Aerial part & W & 2 \\
\hline \multirow[t]{4}{*}{ Brassicaceae } & $\begin{array}{l}\text { Brassica napus L. } \\
\text { (BCN46856) }\end{array}$ & Nap. Nap de bou. Nap del camp & $\begin{array}{l}\text { Boiled in water }{ }^{a,} \\
\text { e. Raw }{ }^{a,} \text { e }\end{array}$ & $\begin{array}{l}\text { Aerial part. } \\
\text { Root. Whole } \\
\text { plant }\end{array}$ & C & 46 \\
\hline & $\begin{array}{l}\text { Brassica oleracea L. } \\
\text { subsp. oleracea } \\
\text { (BCN32181) }\end{array}$ & Bròquil. Col. Col aloma & $\begin{array}{l}\text { Boiled in water }{ }^{a,} \\
\text { e. Raw }{ }^{c,} \text { e, f }\end{array}$ & Leaf & C & 22 \\
\hline & $\begin{array}{l}\text { Capsella bursa-pastoris } \\
\text { (L.) Medic. (BCN46079) }\end{array}$ & Bossa de pastor. Caps blancs & $\operatorname{Raw}^{f}$ & Aerial part & W & 1 \\
\hline & $\begin{array}{l}\text { Raphanus } \\
\text { raphanistrum L. subsp. } \\
\text { sativus (L.) Domin } \\
\text { (BCN49860) }\end{array}$ & Rave & $\operatorname{Raw}^{a}$ & Whole plant & C & 1 \\
\hline Cannabaceae & $\begin{array}{l}\text { Celtis australis L. } \\
\text { (BCN29845) }\end{array}$ & Lledó (fruit). Lledoner & $\begin{array}{l}\text { Boiled in } \\
\text { water }^{\mathrm{e}} \text {. Raw } \\
\text { Ra, e }\end{array}$ & Leaf & C & 10 \\
\hline
\end{tabular}


Table 5 Fodder plants reported in the studied area (Continued)

\begin{tabular}{|c|c|c|c|c|c|c|}
\hline Family & Taxon (voucher) & Catalan vernacular names & Preparation & Used part & $\begin{array}{l}\text { Wild }(W) / \\
\text { cultivated } \\
\text { (C) }\end{array}$ & $\begin{array}{l}\text { Use } \\
\text { reports }\end{array}$ \\
\hline Caprifoliaceae & $\begin{array}{l}\text { Scabiosa atropurpurea } \\
\text { L. (BCN29947) }\end{array}$ & Escabiosa & $\operatorname{Raw}^{f}$ & Aerial part & W & 1 \\
\hline Convolvulaceae & $\begin{array}{l}\text { Convolvulus arvensis L. } \\
\text { (BCN29663) }\end{array}$ & Corretjola & $\operatorname{Raw}^{f}$ & Aerial part & W & 6 \\
\hline \multirow[t]{4}{*}{ Cucurbitaceae } & $\begin{array}{l}\text { Cucumis melo L. } \\
\text { (BCN46851) }\end{array}$ & Meló (fruit) & Raw $^{e}$ & Epicarp. Fruit & $C$ & 6 \\
\hline & $\begin{array}{l}\text { Cucurbita maxima } \\
\text { Duch. in Lam. (BCN-S- } \\
\text { 1499) }\end{array}$ & Rabequet (fruit). Carabassa (fruit) & Boiled in water ${ }^{\mathrm{e}}$ & Fruit & C & 1 \\
\hline & $\begin{array}{l}\text { Cucurbita pepo L. var. } \\
\text { oblonga Link } \\
\text { (BCN29859) }\end{array}$ & Carabassó (fruit) & Raw $^{e}$ & Fruit & C & 1 \\
\hline & $\begin{array}{l}\text { Cucurbita pepo L. var. } \\
\text { pepo (BCN49858) }\end{array}$ & Carbassa (fruit). Carbassera. Rabequet (fruit) & $\begin{array}{l}\text { Boiled in } \\
\text { watere. }^{\text {e. Raw }}{ }^{\text {e }}\end{array}$ & Fruit & C & 12 \\
\hline \multirow[t]{2}{*}{ Equisetaceae } & $\begin{array}{l}\text { Equisetum arvense L. } \\
\text { (BCN24767) }\end{array}$ & Cua de cavall. Sangnua & $\operatorname{Raw}^{f}$ & Aerial part & W & 1 \\
\hline & Equisetum sp. & Cua de cavall. Sangnua & $\operatorname{Raw}^{a, d}$ & Aerial part & W & 3 \\
\hline Ericaceae & $\begin{array}{l}\text { Calluna vulgaris (L.) } \\
\text { Hull (BCN113722) }\end{array}$ & Bronsa. Bronsó & $\operatorname{Raw}^{a}$ & Aerial part & W & 1 \\
\hline \multirow[t]{12}{*}{ Fabaceae } & $\begin{array}{l}\text { Ceratonia siliqua L. } \\
\text { (BCN32177) }\end{array}$ & Garrofa (fruit) & Air-dried ${ }^{a, d}$ & Fruit & C & 8 \\
\hline & $\begin{array}{l}\text { Lupinus albus L. } \\
\text { (BCN64375) }\end{array}$ & Llobí & $\operatorname{Raw}^{a, e}$ & Seed & C & 3 \\
\hline & $\begin{array}{l}\text { Medicago sativa L. } \\
\text { (BCN29891) }\end{array}$ & Userda & $\begin{array}{l}\text { Air-dried }{ }^{a, b}, d, e, \\
\text { f. Boiled in } \\
\text { water }^{a} \text {. Raw }{ }^{b, f}\end{array}$ & Aerial part & C & 34 \\
\hline & $\begin{array}{l}\text { Onobrychis viciifolia } \\
\text { Scop. (BCN113732) }\end{array}$ & Esparcet. Trepadella & $\begin{array}{l}\text { Air-dried }{ }^{a, e, ~ f, ~ g . ~} \\
\text { Raw }^{f} \text { Not } \\
\text { reported }^{f}\end{array}$ & Aerial part & C & 20 \\
\hline & $\begin{array}{l}\text { Pisum sativum L. } \\
\text { (BCN32140) }\end{array}$ & Pèsol & $\operatorname{Raw}^{c}$ & Fruit & C & 1 \\
\hline & $\begin{array}{l}\text { Robinia pseudoacacia } \\
\text { L. (BCN31298) }\end{array}$ & Acàcia. Acàcia de jardí & $\operatorname{Raw}^{\mathrm{b}}$ & Leaf & C & 1 \\
\hline & $\begin{array}{l}\text { Spartium junceum L. } \\
\text { (BCN29956) }\end{array}$ & Ginesta & $\operatorname{Raw}^{\mathrm{b}}$ & Aerial part & W & 1 \\
\hline & $\begin{array}{l}\text { Trifolium incarnatum L. } \\
\text { (BCN25026) }\end{array}$ & Fenc & $\begin{array}{l}\text { Air-dried }{ }^{a,} \\
\text { d. Boiled in } \\
\text { water }^{a} \text {. Raw }\end{array}$ & Aerial part & C & 23 \\
\hline & $\begin{array}{l}\text { Trifolium pratense } \mathrm{L} . \\
\text { (BCN29811) }\end{array}$ & Trèfola. Trèfoga & Air dried ${ }^{a}$. Raw ${ }^{f}$ & Aerial part & W & 4 \\
\hline & $\begin{array}{l}\text { Trigonella foenum- } \\
\text { graecum L. (BCN32120) }\end{array}$ & Senigrec & Raw & Aerial part & W & 1 \\
\hline & $\begin{array}{l}\text { Vicia faba L. } \\
\text { (BCN46826) }\end{array}$ & Fava & $\begin{array}{l}\text { Air-dried, }{ }^{\text {d, e. }} \text {. } \\
\text { Raw }^{\text {a }}\end{array}$ & Seed & C & 6 \\
\hline & $\begin{array}{l}\text { Vicia sativa L. } \\
\text { (BCN47746) }\end{array}$ & Veça & $\operatorname{Raw}^{a, f}$ & Aerial part & C & 3 \\
\hline \multirow[t]{2}{*}{ Fagaceae } & $\begin{array}{l}\text { Castanea sativa Mill. } \\
\text { (BCN29844) }\end{array}$ & Castanya (fruit). Castanyer & $\operatorname{Raw}^{e}$ & Fruit & C & 1 \\
\hline & $\begin{array}{l}\text { Quercus ilex L. } \\
\text { (BCN113730) }\end{array}$ & Aglà (fruit). Alzina. Aulina. Gla (fruit) & $\begin{array}{l}\text { Air dried }{ }^{\mathrm{e}} \text {. } \\
\text { Boiled in } \\
\text { water }^{\mathrm{e}} \text {. } \\
\text { Cooked } \text {. Raw }^{\mathrm{a}} \\
\mathrm{e}, \mathrm{f}\end{array}$ & $\begin{array}{l}\text { Fruit. Leaf. } \\
\text { Young shoot }\end{array}$ & W & 37 \\
\hline
\end{tabular}


Table 5 Fodder plants reported in the studied area (Continued)

\begin{tabular}{|c|c|c|c|c|c|c|}
\hline Family & Taxon (voucher) & Catalan vernacular names & Preparation & Used part & $\begin{array}{l}\text { Wild }(W) / \\
\text { cultivated } \\
\text { (C) }\end{array}$ & $\begin{array}{l}\text { Use } \\
\text { reports }\end{array}$ \\
\hline & $\begin{array}{l}\text { Quercus pubescens } \\
\text { Willd. (BCN30007) }\end{array}$ & Roure & $\operatorname{Raw}^{a, e}$ & Aerial part & W & 3 \\
\hline & $\begin{array}{l}\text { Quercus suber L. } \\
\text { (BCN46829) }\end{array}$ & Suro. Alzina surera & $\operatorname{Raw}^{a}$ & Leaf & W & 2 \\
\hline Linaceae & $\begin{array}{l}\text { Linum usitatissimum L. } \\
\text { (BCN47281) }\end{array}$ & Farina de llinet (elaborated product). Llinet & Air dried ${ }^{e}$ & Seed & $C$ & 1 \\
\hline Malvaceae & $\begin{array}{l}\text { Malva sylvestris L. } \\
\text { (BCN29889) }\end{array}$ & Malva. Malva rosa & $\operatorname{Raw}^{\mathrm{f}}$ & Leaf & W & 1 \\
\hline Moraceae & $\begin{array}{l}\text { Ficus carica L. } \\
\text { (BCN24887) }\end{array}$ & $\begin{array}{l}\text { Figa (infructescence). Figa d'Alacant } \\
\text { (infructescence). Figa de coll de senyora } \\
\text { (infructescence). Figa de coll llarg blanca } \\
\text { (infructescence). Figa de coll llarg negra } \\
\text { (infructescence). Figa de pota de cavall } \\
\text { (infructescence). Figa de Sant Joan (infructescence). } \\
\text { Figa negra (infructescence). Figuera. Figuera de coll } \\
\text { de senyora }\end{array}$ & Raw $^{e}$ & Infructescence & C & 1 \\
\hline Oleaceae & $\begin{array}{l}\text { Fraxinus excelsior L. } \\
\text { (BCN46844) }\end{array}$ & Freixa & $\operatorname{Raw}^{b, g}$ & Leaf & W & 2 \\
\hline Passifloraceae & $\begin{array}{l}\text { Passiflora caerulea L. } \\
\text { (BCN29747) }\end{array}$ & Flor de crist & $\operatorname{Raw}^{g}$ & Aerial part & C & 1 \\
\hline \multirow[t]{3}{*}{ Plantaginaceae } & $\begin{array}{l}\text { Plantago lanceolata } \mathrm{L} \text {. } \\
\text { (BCN32138) }\end{array}$ & Plantatge de fulla estreta. Plantatge estret & $\operatorname{Raw}^{f}$ & Aerial part & W & 1 \\
\hline & $\begin{array}{l}\text { Plantago major L. } \\
\text { (BCN29910) }\end{array}$ & Plantatge. Plantatge ample. Plantatge de fulla ampla & $\operatorname{Raw}^{f}$ & $\begin{array}{l}\text { Aerial part. } \\
\text { Leaf. Whole } \\
\text { plant }\end{array}$ & W & 6 \\
\hline & Plantago sp. & Plantatge & $\operatorname{Raw}^{f}$ & Leaf & W & 2 \\
\hline \multirow[t]{12}{*}{ Poaceae } & $\begin{array}{l}\text { Arundo donax L. } \\
\text { (BCN29825) }\end{array}$ & Canya. Canya americana. Canyer & $\operatorname{Raw}^{a, b}$ & Leaf & W & 2 \\
\hline & $\begin{array}{l}\text { Avena sativa L. } \\
\text { (BCN29839) }\end{array}$ & Civada & $\begin{array}{l}\text { Air dried }{ }^{a, c, f} . \\
\text { Raw }^{b}\end{array}$ & $\begin{array}{l}\text { Aerial part. } \\
\text { Fruit }\end{array}$ & $C$ & 26 \\
\hline & $\begin{array}{l}\text { Cynodon dactylon (L.) } \\
\text { Pers (BCN29686) }\end{array}$ & Gram & $\operatorname{Raw}^{\mathrm{a}}$ & Aerial part & W & 1 \\
\hline & $\begin{array}{l}\text { Digitaria sanguinalis } \\
\text { (L.) Scop. (BCN113745) }\end{array}$ & Forcadella. Xereix & $\operatorname{Raw}^{f}$ & Aerial part & W & 2 \\
\hline & $\begin{array}{l}\text { Hordeum vulgare L. } \\
\text { (BCN46843) }\end{array}$ & Ordi & $\begin{array}{l}\text { Air dried }{ }^{c, g} \text {. } \\
\text { Boiled in water }\end{array}$ & $\begin{array}{l}\text { Aerial part. } \\
\text { Fruit }\end{array}$ & C & 10 \\
\hline & $\begin{array}{l}\text { Lolium perenne } \mathrm{L} . \\
\text { (BCN58204) }\end{array}$ & Margall. Raigràs & $\begin{array}{l}\text { Air dried } d^{a,} 9 \\
\text { Raw }^{b, f}\end{array}$ & Aerial part & C & 10 \\
\hline & $\begin{array}{l}\text { Panicum miliaceum L. } \\
\text { (BCN12911) }\end{array}$ & Mill & Air dried ${ }^{c}$ & Fruit & $C$ & 5 \\
\hline & $\begin{array}{l}\text { Phalaris arundinacea } \mathrm{L} \text {. } \\
\text { (BCN51675) }\end{array}$ & - & $\operatorname{Raw}^{\mathrm{b}}$ & Aerial part & $C$ & 1 \\
\hline & $\begin{array}{l}\text { Secale cereale } \mathrm{L} . \\
\text { (BCN46828) }\end{array}$ & Sègol. Sègal & $\begin{array}{l}\text { Air dried }{ }^{\mathrm{a}} . \\
\text { Boiled in water }{ }^{\mathrm{a}} \text {. } \\
\text { Raw }^{\mathrm{b}}\end{array}$ & $\begin{array}{l}\text { Aerial part. } \\
\text { Fruit }\end{array}$ & $C$ & 6 \\
\hline & $\begin{array}{l}\text { Sorghum bicolor (L.) } \\
\text { Moench (BCN31310) }\end{array}$ & Melca. Sorgo & $\begin{array}{l}\text { Air dried }{ }^{a, c}, d \\
\operatorname{Raw}^{a}\end{array}$ & Aerial part & C & 6 \\
\hline & $\begin{array}{l}\text { Triticum aestivum L. } \\
\text { (BCN29963) }\end{array}$ & $\begin{array}{l}\text { Blat. Farina (elaborated product). Pa (elaborated } \\
\text { product). Palla (elaborated product). Segó (bran) }\end{array}$ & $\begin{array}{l}\text { Air dried }{ }^{a, b}, c, d, \\
\text { e. Boiled in } \\
\text { water }^{c,} \text { e. } \\
\text { Cooked }^{f}\end{array}$ & $\begin{array}{l}\text { Aerial part. } \\
\text { Bran. Fruit }\end{array}$ & C & 28 \\
\hline & $\begin{array}{l}\text { Zea mays L. } \\
\text { (BCN29830) }\end{array}$ & $\begin{array}{l}\text { Blat de morassa. Blat de moret. Blat de moro. Farro } \\
\text { (elaborated product) }\end{array}$ & $\begin{array}{l}\text { Air dried }{ }^{a, c}, \text { d, e, } \\
\text { f, g . Boiled in } \\
\text { water }^{c,} \text { e. Raw } \\
\text { a }\end{array}$ & $\begin{array}{l}\text { Aerial part. } \\
\text { Bract. Fruit }\end{array}$ & C & 56 \\
\hline
\end{tabular}


Table 5 Fodder plants reported in the studied area (Continued)

\begin{tabular}{|c|c|c|c|c|c|c|}
\hline Family & Taxon (voucher) & Catalan vernacular names & Preparation & Used part & $\begin{array}{l}\text { Wild (W)/ } \\
\text { cultivated } \\
\text { (C) }\end{array}$ & $\begin{array}{l}\text { Use } \\
\text { reports }\end{array}$ \\
\hline Polygonaceae & $\begin{array}{l}\text { Fagopyrum esculentum } \\
\text { Moench (BCN24886) }\end{array}$ & Fajol & Boiled in water & Seed & C & 1 \\
\hline Portulacaceae & $\begin{array}{l}\text { Portulaca oleracea L. } \\
\text { (BCN46835) }\end{array}$ & Verdolaga & $\operatorname{Raw}^{a, e, f}$ & Aerial part & W & 11 \\
\hline Primulaceae & $\begin{array}{l}\text { Anagallis arvensis L. } \\
\text { (BCN29974) }\end{array}$ & Marruc & $\operatorname{Raw}^{c}$ & Aerial part & W & 2 \\
\hline \multirow[t]{4}{*}{ Ranunculaceae } & $\begin{array}{l}\text { Anemone hepatica L. } \\
\text { (BCN29834) }\end{array}$ & Herba fetgera & $\operatorname{Raw}^{\mathrm{c}}$ & Leaf & W & 2 \\
\hline & $\begin{array}{l}\text { Clematis flammula L. } \\
\text { (BCN29856) }\end{array}$ & Viadella. Virobella & Air dried ${ }^{a}$ & Leaf & W & 3 \\
\hline & $\begin{array}{l}\text { Clematis recta L. } \\
\text { (BCN113720) }\end{array}$ & Viadella & Air dried $^{a}$ & Aerial part & W & 1 \\
\hline & $\begin{array}{l}\text { Clematis vitalba L. } \\
\text { (BCN29857) }\end{array}$ & Ridorta & Air dried ${ }^{a} \cdot$ Raw $^{g}$ & Leaf & W & 2 \\
\hline Rosaceae & $\begin{array}{l}\text { Rubus ulmifolius Schott } \\
\text { (BCN29938) }\end{array}$ & Bardissa. Mora (fruit). Mora negra (fruit). Romeguera & $\operatorname{Raw}^{b}$ & Young shoot & W & 1 \\
\hline \multirow[t]{2}{*}{ Smilacaceae } & $\begin{array}{l}\text { Sanguisorba minor } \\
\text { Scop. (BCN113728) }\end{array}$ & Esparcet bord & $\operatorname{Raw}^{\mathrm{a}}$ & Aerial part & w & 1 \\
\hline & $\begin{array}{l}\text { Smilax aspera L. } \\
\text { (BCN29951) }\end{array}$ & Arítjol & $\operatorname{Raw}^{f}$ & Aerial part & W & 3 \\
\hline \multirow[t]{2}{*}{ Solanaceae } & $\begin{array}{l}\text { Solanum lycopersicum } \\
\text { L. (BCN29952) }\end{array}$ & $\begin{array}{l}\text { Tomata (fruit). Tomata de guardar (fruit). Tomata de } \\
\text { la meta (fruit). Tomata de penjar (fruit). Tomata dels } \\
\text { tres cantos (fruit). Tomata plena (fruit). Tomata } \\
\text { poma (fruit) }\end{array}$ & $\operatorname{Raw}^{c}$ & Fruit & C & 1 \\
\hline & $\begin{array}{l}\text { Solanum tuberosum L. } \\
\text { (BCN29797) }\end{array}$ & Patata. Patatera. Trumfera & $\begin{array}{l}\text { Boiled in water }{ }^{c_{1}} \\
\text { e. Raw }\end{array}$ & Tuber & C & 5 \\
\hline Ulmaceae & $\begin{array}{l}\text { Ulmus minor Mill. } \\
\text { (BCN113729) }\end{array}$ & Om & $\begin{array}{l}\text { Boiled in } \\
\text { water }^{e} \text {. Raw }{ }^{a, b} \text {, } \\
e, f\end{array}$ & Leaf & W & 10 \\
\hline
\end{tabular}

Fodder destination: ${ }^{\mathrm{a} C o w s},{ }^{\mathrm{b}}$ Goats, ${ }^{\mathrm{C}}$ Hens, ${ }^{\mathrm{d}}$ Horses and mares, ${ }^{\mathrm{e}}$ Pigs, ${ }^{\mathrm{f}}$ Rabbits, ${ }^{\mathrm{g}}$ Sheep; without superscripted letter: animal destination is not clear

which has been mentioned 52 times with a unique popular name (farigola in Catalan language). In contrast, the species designated with more vernacular names are the cultivated ones, due to the names of landraces of several taxa that have also been quoted by the informants.

The ethnophytonymy index [53] shows a high value (32.6\%), meaning that roughly one-third of the plants in the studied area have at least one folk name, comparable to the results from other Catalan territories, such as 35\% in the Pallars district [5], 31\% in Alt Empordà district [10], 29.8\% in the island of Mallorca [12], 28\% in the Montseny mountain massif [6], and $18 \%$ in Castelló province [1]. The allochthonous ethophytonymy index proposed by Carrió [12] calculates the rate between taxa having a vernacular name in non-Catalan languages (even for those taxa having also some Catalan names) and the total number of collected taxa. In the present study, the value is very small $(4.7 \%$, due to a few Spanish names) as compared with the one obtained in Mallorca (27.8, due to some names in Spanish and French languages; [12]), indicating a more culturally homogeneous informants' pool.

The linguistic diversity index, which expresses the linguistic richness of a territory independently of its flora, reaches a value of 1.90 (almost two names per plant, in mean), comparable to those obtained in l'Alt Empordà (1.94) [10], Navarra (1.87) [75], and Montseny (1.76) [6].

It is interesting to remark that a certain number of folk plant names are linked to their uses. For instance, Achillea ageratum is called in Catalan language herba del fàstic ("disgusting herb"), since it incites vomiting; herba cuquera ("worm's herb") and herba fetgera ("liver's herb") allude, respectively, to the antihelminthic use of Agrimonia eupatoria and the hepatoprotective use of Anemone hepatica (the latter bearing the same indication in its specific epithet); Centaurea aspera, an hypoglycemiant plant, is named flor del sucre ("sugar's flower"); nap de bou ("cow's rape") announces the use of Brassica napus to feed cattle; bruc d'escombres ("broom's heather") 
Table 6 Plant with other uses reported in the studied area

\begin{tabular}{|c|c|c|c|c|c|}
\hline Family & Taxon (voucher) & Catalan vernacular names & Use & Used part & $\begin{array}{l}\text { Use } \\
\text { reports }\end{array}$ \\
\hline \multirow[t]{2}{*}{ Adoxaceae } & $\begin{array}{l}\text { Sambucus nigra L. } \\
\text { (BCN113595) }\end{array}$ & Sabuquer. Saüc. Saüquer & Artisanal. Fuel obtaining & Stem & 14 \\
\hline & $\begin{array}{l}\text { Viburnum tinus L. } \\
\text { (BCN30012) }\end{array}$ & Marfull & Ornamental & Whole plant & 1 \\
\hline Apiaceae & $\begin{array}{l}\text { Pimpinella anisum L. } \\
\text { (BCN47278) }\end{array}$ & Anís verd. Matafaluga & Repellent & Whole plant & 1 \\
\hline Araceae & $\begin{array}{l}\text { Arum italicum Mill. } \\
\text { (BCN32358) }\end{array}$ & Xàrria. Xèrria & Agrosilvopastoral management & Flower & 1 \\
\hline Araliaceae & $\begin{array}{l}\text { Hedera helix L. } \\
\text { (BCN29869) }\end{array}$ & Heura. Heura d'alzina & Ornamental & Whole plant & 1 \\
\hline Arecaceae & $\begin{array}{l}\text { Phoenix dactylifera } \\
\text { L. (BCN52783) }\end{array}$ & Palma & Magic and religious beliefs and practices & Leaf & 5 \\
\hline \multirow[t]{4}{*}{ Asparagaceae } & $\begin{array}{l}\text { Agave americana L. } \\
\text { (BCN46860) }\end{array}$ & Figuerassa & Unclassified & Inflorescence & 1 \\
\hline & $\begin{array}{l}\text { Asparagus } \\
\text { acutifolius L. } \\
\text { (BCN29976) }\end{array}$ & Espàrgol. Esparreguera. Espàrrec & Folk oral literature. Ornamental & $\begin{array}{l}\text { Aerial part. Young } \\
\text { shoot }\end{array}$ & 2 \\
\hline & $\begin{array}{l}\text { Ruscus aculeatus L. } \\
\text { (BCN29939) }\end{array}$ & Galzeran. Galleranc & Ornamental & Aerial part & 3 \\
\hline & $\begin{array}{l}\text { Yucca aloifolia L. } \\
\text { (BCN286) }\end{array}$ & - & Ornamental. Unclassified & Whole plant & 2 \\
\hline \multirow[t]{6}{*}{ Asteraceae } & $\begin{array}{l}\text { Carlina acanthifolia } \\
\text { All. (BCN24738) }\end{array}$ & Cardina. Carlina. Carolina & Domestic. Ornamental & Whole plant & 8 \\
\hline & $\begin{array}{l}\text { Helichrysum } \\
\text { stoechas (L.) } \\
\text { Moench } \\
\text { (BCN29872) }\end{array}$ & Mançanilla. Sempreviva & Ornamental & Aerial part & 2 \\
\hline & $\begin{array}{l}\text { Mantisalca } \\
\text { salmantica (L.) Briq. } \\
\text { et Cavill. } \\
\text { (BCN24925) }\end{array}$ & Baleja & Artisanal & Aerial part & 1 \\
\hline & $\begin{array}{l}\text { Santolina } \\
\text { chamaecyparissus L. } \\
\text { (BCN113709) }\end{array}$ & Espernallac. Santolina & Ornamental & Whole plant & 1 \\
\hline & Sonchus sp. & Llepsó. Lletissó. Llistó & $\begin{array}{l}\text { Agrosilvopastoral management. } \\
\text { Unclassified }\end{array}$ & $\begin{array}{l}\text { Aerial part. Whole } \\
\text { plant }\end{array}$ & 2 \\
\hline & $\begin{array}{l}\text { Taraxacum officinale } \\
\text { Weber in Wiggers } \\
\text { (BCN25948) }\end{array}$ & Dent de lleó. Xicoia & Ludic & Infructescence & 2 \\
\hline Begoniaceae & Begonia sp. & Tamaia & Ornamental & Whole plant & 1 \\
\hline \multirow[t]{2}{*}{ Betulaceae } & $\begin{array}{l}\text { Alnus glutinosa (L.) } \\
\text { Gaertn. (BCN29620) }\end{array}$ & Vern & Artisanal. Timber & Stem & 4 \\
\hline & $\begin{array}{l}\text { Corylus avellana L. } \\
\text { (BCN29831) }\end{array}$ & $\begin{array}{l}\text { Avellana (fruit). Avellana del queixal (fruit). Avellaner. } \\
\text { Avellaner negret }\end{array}$ & $\begin{array}{l}\text { Agrosilvopastoral management. Artisanal. } \\
\text { Fuel obtaining. Timber }\end{array}$ & Stem & 15 \\
\hline Boraginaceae & $\begin{array}{l}\text { Lithospermum } \\
\text { officinale L. } \\
\text { (BCN113576) }\end{array}$ & Herba pedrera & Ornamental & Aerial part & 1 \\
\hline Buxaceae & $\begin{array}{l}\text { Buxus sempervirens } \\
\text { L. (BCN29843) }\end{array}$ & Boix & $\begin{array}{l}\text { Agrosilvopastoral management. Artisanal. } \\
\text { Timber }\end{array}$ & Aerial part. Stem & 14 \\
\hline \multirow[t]{2}{*}{ Cannabaceae } & $\begin{array}{l}\text { Cannabis sativa } \mathrm{L} \text {. } \\
\text { (BCN24735) }\end{array}$ & Cànem. Carm & Artisanal. Textile & Stem & 6 \\
\hline & $\begin{array}{l}\text { Celtis australis L. } \\
\text { (BCN29845) }\end{array}$ & Lledó (fruit). Lledoner & $\begin{array}{l}\text { Agrosilvopastoral management. Artisanal. } \\
\text { Timber }\end{array}$ & Fruit. Stem & 63 \\
\hline \multirow[t]{3}{*}{ Cistaceae } & $\begin{array}{l}\text { Cistus albidus L. } \\
\text { (BCN36672) }\end{array}$ & Estepa & Smoking plant & Leaf & 1 \\
\hline & $\begin{array}{l}\text { Cistus monspeliensis } \\
\text { L. (BCN36740) }\end{array}$ & Estepa. Mòdega & Artisanal & Aerial part & 2 \\
\hline & $\begin{array}{l}\text { Cistus salviifolius L. } \\
\text { (BCN36767) }\end{array}$ & Estepa. Mòdega & Artisanal. Domestic & Aerial part & 4 \\
\hline Coriariaceae & Coriaria myrtifolia L. & Roldor & Agrosilvopastoral management. Artisanal. & Aerial part. Stem & 7 \\
\hline
\end{tabular}


Table 6 Plant with other uses reported in the studied area (Continued)

\begin{tabular}{|c|c|c|c|c|c|}
\hline Family & Taxon (voucher) & Catalan vernacular names & Use & Used part & $\begin{array}{l}\text { Use } \\
\text { reports }\end{array}$ \\
\hline & (BCN113731) & & Magic and religious beliefs and practices & & \\
\hline Cucurbitaceae & $\begin{array}{l}\text { Cucurbita pepo L. } \\
\text { var. pepo } \\
\text { (BCN49858) }\end{array}$ & Carbassa (fruit). Carbassera. Rabequet (fruit) & Artisanal & Fruit & 1 \\
\hline \multirow[t]{3}{*}{ Cupressaceae } & $\begin{array}{l}\text { Cupressus } \\
\text { sempervirens L. } \\
\text { (BCN35770) }\end{array}$ & Xiprer & Folk oral literature & Whole plant & 2 \\
\hline & $\begin{array}{l}\text { Juniperus communis } \\
\text { L. (BCN113589) }\end{array}$ & Ginebre. Ginebró & Timber & Stem & 1 \\
\hline & $\begin{array}{l}\text { Juniperus oxycedrus } \\
\text { L. (BCN29879) }\end{array}$ & Càdec & Artisanal. Domestic. Timber & Fruit. Stem & 4 \\
\hline Dennstaedtiaceae & $\begin{array}{l}\text { Pteridium aquilinum } \\
\text { (L.) Kuhn } \\
\text { (BCN113735) }\end{array}$ & Falguera & Agrosilvopastoral management & Frond & 5 \\
\hline Dryopteridaceae & $\begin{array}{l}\text { Dryopteris filix-mas } \\
\text { (L.) Schott } \\
\text { (BCN29629) }\end{array}$ & Falguera & Agrosilvopastoral management & Frond & 8 \\
\hline \multirow[t]{2}{*}{ Equisetaceae } & $\begin{array}{l}\text { Equisetum arvense L. } \\
\text { (BCN24767) }\end{array}$ & Cua de cavall. Sangnua & Agrosilvopastoral management & Aerial part & 1 \\
\hline & Equisetum sp. & Cua de cavall. Sangnua & Agrosilvopastoral management & Aerial part & 1 \\
\hline \multirow[t]{6}{*}{ Ericaceae } & $\begin{array}{l}\text { Arbutus unedo L. } \\
\text { (BCN29836) }\end{array}$ & $\begin{array}{l}\text { Arboç. Bola d'arboç (fruit). Cirera d'arboç (fruit). } \\
\text { Cirerer d'arboç }\end{array}$ & $\begin{array}{l}\text { Agrosilvopastoral management. Artisanal. } \\
\text { Fuel obtaining. Not reported }\end{array}$ & Stem & 11 \\
\hline & $\begin{array}{l}\text { Calluna vulgaris (L.) } \\
\text { Hull (BCN113722) }\end{array}$ & Bronsa. Bronsó & $\begin{array}{l}\text { Agrosilvopastoral management. Fuel } \\
\text { obtaining }\end{array}$ & Aerial part & 4 \\
\hline & $\begin{array}{l}\text { Erica arborea L. } \\
\text { (BCN113593) }\end{array}$ & $\begin{array}{l}\text { Bruc. Bruc boal. Bruc bord. Bruc d'ull de bou. Bruc de } \\
\text { bou. Bruc de llei }\end{array}$ & $\begin{array}{l}\text { Agrosilvopastoral management. Artisanal. } \\
\text { Fuel obtaining. Timber. Unclassified }\end{array}$ & $\begin{array}{l}\text { Aerial part. Root. } \\
\text { Stem }\end{array}$ & 38 \\
\hline & $\begin{array}{l}\text { Erica multiflora L. } \\
\text { (BCN29864) }\end{array}$ & Bruc & $\begin{array}{l}\text { Agrosilvopastoral management. Artisanal. } \\
\text { Fuel obtaining. Unclassified }\end{array}$ & Aerial part & 4 \\
\hline & $\begin{array}{l}\text { Erica scoparia L. } \\
\text { (BCN113724) }\end{array}$ & Bruc. Bruc bord. Bruc d'escombres. Bruc de llei & $\begin{array}{l}\text { Agrosilvopastoral management. Artisanal. } \\
\text { Domestic. Fuel obtaining. Unclassified }\end{array}$ & $\begin{array}{l}\text { Aerial part. Stem. } \\
\text { Whole plant }\end{array}$ & 44 \\
\hline & Erica sp. & Bruc & $\begin{array}{l}\text { Agrosilvopastoral management. Artisanal. } \\
\text { Fuel obtaining. }\end{array}$ & Aerial part. Stem & 8 \\
\hline \multirow[t]{11}{*}{ Fabaceae } & $\begin{array}{l}\text { Genista scorpius (L.) } \\
\text { DC. in Lam. et DC. } \\
\text { (BCN27292) }\end{array}$ & Argelaga. Espines & $\begin{array}{l}\text { Agrosilvopastoral management. Fuel } \\
\text { obtaining. }\end{array}$ & Aerial part & 13 \\
\hline & $\begin{array}{l}\text { Medicago sativa L. } \\
\text { (BCN29891) }\end{array}$ & Userda & Agrosilvopastoral management & Aerial part & 1 \\
\hline & $\begin{array}{l}\text { Onobrychis viciifolia } \\
\text { Scop. (BCN113732) }\end{array}$ & Esparcet. Trepadella & Agrosilvopastoral management & Flower & 1 \\
\hline & $\begin{array}{l}\text { Phaseolus vulgaris } L \text {. } \\
\text { (BCN46837) }\end{array}$ & $\begin{array}{l}\text { Fesol de l'ull marrón. Fesol de l'ull negre. Fesol de } \\
\text { Santa Pau. Fesol del bitxet gros. Fesol de bitxet petit. } \\
\text { Fesol menut. Fesola. Mongeta }\end{array}$ & Agrosilvopastoral management & Whole plant & 1 \\
\hline & $\begin{array}{l}\text { Robinia } \\
\text { pseudoacacia L. } \\
\text { (BCN31298) }\end{array}$ & Acàcia. Acàcia de jardí & $\begin{array}{l}\text { Agrosilvopastoral management. } \\
\text { Ornamental. Timber }\end{array}$ & Stem. Whole plant & 13 \\
\hline & $\begin{array}{l}\text { Spartium junceum L. } \\
\text { (BCN29956) }\end{array}$ & Ginesta & $\begin{array}{l}\text { Agrosilvopastoral management. Fuel } \\
\text { obtaining. Magic and religious beliefs } \\
\text { and practices. Timber }\end{array}$ & $\begin{array}{l}\text { Aerial part. Flower. } \\
\text { Stem }\end{array}$ & 12 \\
\hline & $\begin{array}{l}\text { Trifolium } \\
\text { incarnatum L. } \\
\text { (BCN25026) }\end{array}$ & Fenc & Agrosilvopastoral management & Aerial part. Flower & 2 \\
\hline & $\begin{array}{l}\text { Trigonella foenum- } \\
\text { graecum L. } \\
\text { (BCN32120) }\end{array}$ & Senigrec & Repellent & Whole plant & 1 \\
\hline & $\begin{array}{l}\text { Ulex parviflorus } \\
\text { Pourr. (BCN30011) }\end{array}$ & Gatosa & $\begin{array}{l}\text { Agrosilvopastoral management. Fuel } \\
\text { obtaining }\end{array}$ & Aerial part & 3 \\
\hline & $\begin{array}{l}\text { Wisteria sinensis } \\
\text { (Sims) Sweet } \\
\text { (BCN30014) }\end{array}$ & Lilà & Ornamental & Whole plant & 1 \\
\hline & Castanea sativa Mill. & Castanya (fruit). Castanyer & Agrosilvopastoral management. Artisanal. & Stem & 28 \\
\hline
\end{tabular}


Table 6 Plant with other uses reported in the studied area (Continued)

\begin{tabular}{|c|c|c|c|c|c|}
\hline Family & Taxon (voucher) & Catalan vernacular names & Use & Used part & $\begin{array}{l}\text { Use } \\
\text { reports }\end{array}$ \\
\hline & (BCN29844) & & Timber & & \\
\hline \multirow[t]{5}{*}{ Fagaceae } & $\begin{array}{l}\text { Fagus sylvatica L. } \\
\text { (BCN46845) }\end{array}$ & Faig & Fuel obtaining & Stem & 1 \\
\hline & $\begin{array}{l}\text { Quercus coccifera L. } \\
\text { (BCN29765) }\end{array}$ & Garrigues & Domestic & Aerial part & 1 \\
\hline & $\begin{array}{l}\text { Quercus ilex L. } \\
\text { (BCN113730) }\end{array}$ & Aglà (fruit). Alzina. Aulina. Gla (fruit) & $\begin{array}{l}\text { Agrosilvopastoral management. Artisanal. } \\
\text { Dyer. Fuel obtaining. Ludic. Magic and } \\
\text { religious beliefs and practices. Not } \\
\text { reported. Tannery. Timber. Unclassified }\end{array}$ & $\begin{array}{l}\text { Aerial part. Bark. } \\
\text { Flower. Fruit. Leaf. } \\
\text { Stem }\end{array}$ & 66 \\
\hline & $\begin{array}{l}\text { Quercus pubescens } \\
\text { Willd. (BCN30007) }\end{array}$ & Roure & $\begin{array}{l}\text { Agrosilvopastoral management. Folk oral } \\
\text { literature. Fuel obtaining. Timber }\end{array}$ & Fruit. Leaf. Stem & 25 \\
\hline & $\begin{array}{l}\text { Quercus suber L. } \\
\text { (BCN46829) }\end{array}$ & Suro. Alzina surera & $\begin{array}{l}\text { Agrosilvopastoral management. } \\
\text { Domestic. Fuel obtaining. Ludic. Textile. } \\
\text { Timber. Unclassified. }\end{array}$ & $\begin{array}{l}\text { Bark. Stem. Whole } \\
\text { plant }\end{array}$ & 15 \\
\hline Geraniaceae & Pelargonium sp. & Gerani & Ornamental & Whole plant & 1 \\
\hline Juglandaceae & $\begin{array}{l}\text { Juglans regia L. } \\
\text { (BCN29877) }\end{array}$ & Noguer. Nou (fruit). Nou verda (fruit) & $\begin{array}{l}\text { Dyer. Folk oral literature. Magic and } \\
\text { religious beliefs and practices }\end{array}$ & Fruit. Whole plant & 5 \\
\hline Juncaceae & $\begin{array}{l}\text { Juncus effusus L. } \\
\text { (BCN39991) }\end{array}$ & Jonc & Agrosilvopastoral management & Stem & 1 \\
\hline \multirow[t]{8}{*}{ Lamiaceae } & $\begin{array}{l}\text { Lavandula latifolia } \\
\text { Medic. (BCN113740) }\end{array}$ & Barballó. Espígol. Lavanda & Cosmetic. Domestic. Ornamental & $\begin{array}{l}\text { Aerial part. Whole } \\
\text { plant }\end{array}$ & 5 \\
\hline & $\begin{array}{l}\text { Ocimum basilicum } \\
\text { L. (BCN29897) }\end{array}$ & Alfàbrega & Agrosilvopastoral management. Repellent & Whole plant & 4 \\
\hline & $\begin{array}{l}\text { Origanum vulgare L. } \\
\text { (BCN113705) }\end{array}$ & Orenga & Folk oral literature & Whole plant & 2 \\
\hline & $\begin{array}{l}\text { Rosmarinus } \\
\text { officinalis L. } \\
\text { (BCN113599) }\end{array}$ & Romaní & $\begin{array}{l}\text { Domestic. Folk oral literature. Magic and } \\
\text { religious beliefs and practices }\end{array}$ & Aerial part & 14 \\
\hline & $\begin{array}{l}\text { Salvia farinacea } \\
\text { Benth. (BCN113718) }\end{array}$ & Sàlvia de jardí & Ornamental & Whole plant & 1 \\
\hline & $\begin{array}{l}\text { Salvia officinalis L. } \\
\text { subsp. officinalis } \\
\text { (BCN113583) }\end{array}$ & Sàlvia. Sàlvia de fulla ampla & Ornamental & Whole plant & 1 \\
\hline & $\begin{array}{l}\text { Thymus xcitriodorus } \\
\text { (Pers.) Schreber } \\
\text { (BCN113803) }\end{array}$ & Farigola de xocolata. Farigola llimonera & Ornamental & Whole plant & 1 \\
\hline & $\begin{array}{l}\text { Thymus vulgaris } \mathrm{L} \text {. } \\
\text { (BCN113590) }\end{array}$ & Farigola & $\begin{array}{l}\text { Folk oral literature. Magic and religious } \\
\text { beliefs and practices }\end{array}$ & $\begin{array}{l}\text { Aerial part. Whole } \\
\text { plant }\end{array}$ & 4 \\
\hline Lauraceae & $\begin{array}{l}\text { Laurus nobilis L. } \\
\text { (BCN113717) }\end{array}$ & Llord. Llorer & Magic and religious beliefs and practices & Aerial part & 39 \\
\hline Lythraceae & $\begin{array}{l}\text { Punica granatum L. } \\
\text { (BCN29764) }\end{array}$ & $\begin{array}{l}\text { Magrana (fruit). Magraner. Magraner agre. Magraner } \\
\text { bord. Magraner dolç }\end{array}$ & $\begin{array}{l}\text { Artisanal. Magic and religious beliefs and } \\
\text { practices }\end{array}$ & Aerial part & 21 \\
\hline \multirow[t]{3}{*}{ Moraceae } & $\begin{array}{l}\text { Ficus carica L. } \\
\text { (BCN24887) }\end{array}$ & $\begin{array}{l}\text { Figa (infructescence). Figa d'Alacant (infructescence). } \\
\text { Figa de coll de senyora (infructescence). Figa de coll } \\
\text { llarg blanca (infructescence). Figa de coll llarg negra } \\
\text { (infructescence). Figa de pota de cavall } \\
\text { (infructescence). Figa de Sant Joan (infructescence). } \\
\text { Figa negra (infructescence). Figuera. Figuera de coll } \\
\text { de senyora }\end{array}$ & $\begin{array}{l}\text { Magic and religious beliefs and practices. } \\
\text { Folk oral literature }\end{array}$ & $\begin{array}{l}\text { Infructescence. } \\
\text { Whole plant }\end{array}$ & 7 \\
\hline & $\begin{array}{l}\text { Morus alba L. } \\
\text { (BCN52588) }\end{array}$ & Morera & Agrosilvopastoral management & Stem & 2 \\
\hline & $\begin{array}{l}\text { Morus nigra L. } \\
\text { (BCN31289) }\end{array}$ & Arça. Morera & $\begin{array}{l}\text { Agrosilvopastoral management. Fuel } \\
\text { obtaining }\end{array}$ & Stem & 3 \\
\hline Myrtaceae & $\begin{array}{l}\text { Eucalyptus globulus } \\
\text { Labill. (BCN29696) }\end{array}$ & Eucaliptu. Eucaliptus & Ornamental & Aerial part & 1 \\
\hline \multirow[t]{2}{*}{ Oleaceae } & $\begin{array}{l}\text { Fraxinus excelsior } \mathrm{L} \text {. } \\
\text { (BCN46844) }\end{array}$ & Freixa & Timber & Stem & 3 \\
\hline & $\begin{array}{l}\text { Olea europaea L. } \\
\text { subsp. europaea } \\
\text { (BCN29898) }\end{array}$ & $\begin{array}{l}\text { Oli (elaborated product). Oli d'oliva (elaborated } \\
\text { product). Olivera. Oliva (fruit) }\end{array}$ & $\begin{array}{l}\text { Artisanal. Domestic. Folk oral literature. } \\
\text { Fuel obtaining. Magic and religious } \\
\text { beliefs and practices. Timber }\end{array}$ & $\begin{array}{l}\text { Aerial part. Fruit. } \\
\text { Stem }\end{array}$ & 10 \\
\hline
\end{tabular}


Table 6 Plant with other uses reported in the studied area (Continued)

\begin{tabular}{|c|c|c|c|c|c|}
\hline Family & Taxon (voucher) & Catalan vernacular names & Use & Used part & $\begin{array}{l}\text { Use } \\
\text { reports }\end{array}$ \\
\hline Papaveraceae & $\begin{array}{l}\text { Papaver rhoeas L. } \\
\text { (BCN29903) }\end{array}$ & Gallaret. Pipiripip. Quiquiriquí. Rosella & $\begin{array}{l}\text { Ludic. Magic and religious beliefs and } \\
\text { practices }\end{array}$ & Flower & 13 \\
\hline \multirow[t]{4}{*}{ Pinaceae } & $\begin{array}{l}\text { Pinus halepensis } \\
\text { Mill. (BCN113592) }\end{array}$ & $\begin{array}{l}\text { Pi. Pi blanc. Pi bord. Pi de pinya llarga. Pi petit. Pinya } \\
\text { (fructification) }\end{array}$ & $\begin{array}{l}\text { Agrosilvopastoral management. Artisanal. } \\
\text { Folk oral literature. Fuel obtaining. Timber }\end{array}$ & $\begin{array}{l}\text { Aerial part. Bark. } \\
\text { Fructification. Stem. } \\
\text { Whole plant }\end{array}$ & 20 \\
\hline & $\begin{array}{l}\text { Pinus pinaster Ait. } \\
\text { (BCN36559) }\end{array}$ & Pi bord. Pi melis & $\begin{array}{l}\text { Agrosilvopastoral management. Fuel } \\
\text { obtaining }\end{array}$ & Stem & 4 \\
\hline & $\begin{array}{l}\text { Pinus pinea L. } \\
\text { (BCN26751) }\end{array}$ & Pi. Pi de llei. Pi de pinya. Pi pinyer & Fuel obtaining & Fructification & 1 \\
\hline & Pinus sp. & Pi. Trementina (elaborated product) & $\begin{array}{l}\text { Agrosilvopastoral management. Artisanal. } \\
\text { Domestic. Fuel obtaining. Timber }\end{array}$ & $\begin{array}{l}\text { Aerial part. Cortical } \\
\text { parenchyma. Leaf. } \\
\text { Stem }\end{array}$ & 9 \\
\hline \multirow[t]{11}{*}{ Poaceae } & $\begin{array}{l}\text { Arundo donax L. } \\
\text { (BCN29825) }\end{array}$ & Canya. Canya americana. Canyer & Agrosilvopastoral management. Artisanal & $\begin{array}{l}\text { Leaf. Stem. Whole } \\
\text { plant }\end{array}$ & 48 \\
\hline & $\begin{array}{l}\text { Avena barbata Pott } \\
\text { ex Link in Schrad. } \\
\text { (BCN49867) }\end{array}$ & Avena. Cugula & Ludic & Fruit & 16 \\
\hline & $\begin{array}{l}\text { Avena sativa L. } \\
\text { (BCN29839) }\end{array}$ & Civada & Agrosilvopastoral management. Ludic & Aerial part. Fruit & 5 \\
\hline & $\begin{array}{l}\text { Briza media L. } \\
\text { (BCN113733) }\end{array}$ & Belluguets & Ornamental & Flower & 2 \\
\hline & $\begin{array}{l}\text { Panicum miliaceum } \\
\text { L. (BCN12911) }\end{array}$ & Mill & Magic and religious beliefs and practices & Fruit & 1 \\
\hline & $\begin{array}{l}\text { Phragmites australis } \\
\text { (Cav.) Steudel } \\
\text { (BCN27104) }\end{array}$ & - & Artisanal & Stem & 1 \\
\hline & $\begin{array}{l}\text { Secale cereale L. } \\
\text { (BCN46828) }\end{array}$ & Sègol. Sègal & Agrosilvopastoral management & Aerial part & 2 \\
\hline & $\begin{array}{l}\text { Sorghum bicolor (L.) } \\
\text { Moench } \\
\text { (BCN31310) }\end{array}$ & Melca. Sorgo & Artisanal & Aerial part & 2 \\
\hline & $\begin{array}{l}\text { Stipa tenacissima } \mathrm{L} . \\
\text { (BCN46091) }\end{array}$ & Espart & Textile & Aerial part. Stem & 3 \\
\hline & $\begin{array}{l}\text { Triticum aestivum L. } \\
\text { (BCN29963) }\end{array}$ & $\begin{array}{l}\text { Blat. Farina (elaborated product). Pa (elaborated } \\
\text { product). Palla (elaborated product). Segó (bran) }\end{array}$ & Agrosilvopastoral management & Fruit & 6 \\
\hline & $\begin{array}{l}\text { Zea mays L. } \\
\text { (BCN29830) }\end{array}$ & $\begin{array}{l}\text { Blat de morassa. Blat de moret. Blat de moro. Farro } \\
\text { (elaborated product) }\end{array}$ & $\begin{array}{l}\text { Agrosilvopastoral management. Artisanal. } \\
\text { Domestic. Folk oral literature. Fuel } \\
\text { obtaining. Ludic. Ornamental. Textile }\end{array}$ & $\begin{array}{l}\text { Bract. Fruit. } \\
\text { Inflorescence.Stem. } \\
\text { Styles and stigmas }\end{array}$ & 31 \\
\hline \multirow[t]{2}{*}{ Ranunculaceae } & $\begin{array}{l}\text { Clematis flammula } \\
\text { L. (BCN29856) }\end{array}$ & Viadella. Virobella & Agrosilvopastoral management & Aerial part & 2 \\
\hline & $\begin{array}{l}\text { Clematis vitalba L. } \\
\text { (BCN29857) }\end{array}$ & Ridorta & $\begin{array}{l}\text { Agrosilvopastoral management. } \\
\text { Domestic. Textile }\end{array}$ & Aerial part. Stem & 6 \\
\hline \multirow[t]{7}{*}{ Rosaceae } & $\begin{array}{l}\text { Crataegus } \\
\text { monogyna Jacq. } \\
\text { (BCN29858) }\end{array}$ & Arç. Arç blanc & $\begin{array}{l}\text { Agrosilvopastoral management. Fuel } \\
\text { obtaining }\end{array}$ & Stem. Whole plant & 5 \\
\hline & $\begin{array}{l}\text { Cydonia oblonga } \\
\text { Mill. (BCN46849) }\end{array}$ & $\begin{array}{l}\text { Codony (fruit). Codonyat (elaborated product). } \\
\text { Codonyer }\end{array}$ & Agrosilvopastoral management & Whole plant & 3 \\
\hline & $\begin{array}{l}\text { Mespilus germanica } \\
\text { L. (BCN50768) }\end{array}$ & Nespler. Nespla de bosc. Nespra. Nespro & Agrosilvopastoral management & Whole plant & 1 \\
\hline & $\begin{array}{l}\text { Prunus armeniaca L. } \\
\text { (BCN48712) }\end{array}$ & Abricoc (fruit). Albercoc (fruit). Albercoquer & Agrosilvopastoral management. Artisanal & $\begin{array}{l}\text { Endocarp. Whole } \\
\text { plant }\end{array}$ & 3 \\
\hline & $\begin{array}{l}\text { Prunus avium (L.) L. } \\
\text { (BCN29827) }\end{array}$ & Cirera (fruit). Cirerer & Agrosilvopastoral management & Stem. Whole plant & 2 \\
\hline & $\begin{array}{l}\text { Prunus dulcis (Mill.) } \\
\text { Weeb. (BCN46833) }\end{array}$ & Ametller & Agrosilvopastoral management & Whole plant & 1 \\
\hline & $\begin{array}{l}\text { Prunus persica }(\mathrm{L} .) \\
\text { Batsch (BCN46832) }\end{array}$ & $\begin{array}{l}\text { Préssec (fruit). Préssec cardinal (fruit). Préssec de } \\
\text { coure (fruit). Préssec duran (fruit). Préssec groc (fruit). } \\
\text { Préssec groc d'agost (fruit). Préssec mollar (fruit). } \\
\text { Préssec sang de llebre (fruit). Préssec de Sant Joan } \\
\text { (fruit). Préssec de Sant Pere (fruit). Presseguer }\end{array}$ & Agrosilvopastoral management & Whole plant & 3 \\
\hline
\end{tabular}


Table 6 Plant with other uses reported in the studied area (Continued)

\begin{tabular}{|c|c|c|c|c|c|}
\hline Family & Taxon (voucher) & Catalan vernacular names & Use & Used part & $\begin{array}{l}\text { Use } \\
\text { reports }\end{array}$ \\
\hline & $\begin{array}{l}\text { Prunus spinosa L. } \\
\text { (BCN30005) }\end{array}$ & Aranyó (fruit). Aranyoner. Arç. Arç negre. Arça & Domestic & Whole plant & 1 \\
\hline & $\begin{array}{l}\text { Pyrus communis L. } \\
\text { subsp. communis } \\
\text { (BCN46831) }\end{array}$ & $\begin{array}{l}\text { Pera (fruit). Pera conference (fruit). Pera de Sant Joan } \\
\text { (fruit). Pera rogija (fruit). Perer. Perer mau }\end{array}$ & Agrosilvopastoral management & Whole plant & 2 \\
\hline & $\begin{array}{l}\text { Pyrus malus L. } \\
\text { subsp. mitis (Wallr.) } \\
\text { O.Bolòs et J.Vigo } \\
\text { (BCN46830) }\end{array}$ & $\begin{array}{l}\text { Poma (fruit). Poma aspra (fruit). Poma cambusina } \\
\text { (fruit). Poma camosa (fruit). Poma capçana (fruit). } \\
\text { Poma del ciri (fruit). Poma del ciri groga (fruit). Poma } \\
\text { del ciri vermella (fruit). Poma golden (fruit). Poma } \\
\text { rodona (fruit). Poma royal (fruit). Pomer. Pomer del } \\
\text { ciri. Pomera. Pomera del ciri }\end{array}$ & Agrosilvopastoral management. Domestic & Fruit. Whole plant & 11 \\
\hline & Rosa sp. & Rosa. Rosa de jardí. Roser & Ornamental & Whole plant & 1 \\
\hline & $\begin{array}{l}\text { Rubus ulmifolius } \\
\text { Schott (BCN29938) }\end{array}$ & Bardissa. Mora (fruit). Mora negra (fruit). Romeguera & Folk oral literature. Fuel obtaining & $\begin{array}{l}\text { Aerial part. Young } \\
\text { shoot }\end{array}$ & 2 \\
\hline \multirow[t]{5}{*}{ Rutaceae } & $\begin{array}{l}\text { Citrus aurantium L. } \\
\text { (BCN46080) }\end{array}$ & $\begin{array}{l}\text { Taronger agre. Taronger amarg. Taronger bord. } \\
\text { Taronja agra (fruit) }\end{array}$ & Agrosilvopastoral management & Fruit. Whole plant & 3 \\
\hline & $\begin{array}{l}\text { Citrus japonica } \\
\text { Thunb. } \\
\text { (BCN113966) }\end{array}$ & Llimona de Xipre & Ornamental & Whole plant & 1 \\
\hline & $\begin{array}{l}\text { Citrus limon (L.) } \\
\text { Burm. (BCN46853) }\end{array}$ & Llimona (fruit). Llimoner & Agrosilvopastoral management & Fruit & 1 \\
\hline & $\begin{array}{l}\text { Citrus sinensis (L.) } \\
\text { Osbeck (BCN24752) }\end{array}$ & Taronger. Taronger dolç. Taronja (fruit) & Agrosilvopastoral management & Whole plant & 1 \\
\hline & $\begin{array}{l}\text { Ruta chalepensis L. } \\
\text { (BCN29940) }\end{array}$ & Ruda & $\begin{array}{l}\text { Domestic. Folk oral literature. Magic and } \\
\text { religious beliefs and practices }\end{array}$ & $\begin{array}{l}\text { Aerial part. Whole } \\
\text { plant }\end{array}$ & 3 \\
\hline \multirow[t]{4}{*}{ Salicaceae } & $\begin{array}{l}\text { Populus } \\
\text { xcanadensis } \\
\text { Moench } \\
\text { (BCN113967) }\end{array}$ & Arbre. Pollancre & Timber & Stem & 1 \\
\hline & $\begin{array}{l}\text { Populus nigra L. } \\
\text { (BCN113746) }\end{array}$ & Arbre. Arbre bord. Pollancre & $\begin{array}{l}\text { Agrosilvopastoral management. Artisanal. } \\
\text { Timber }\end{array}$ & Stem & 3 \\
\hline & $\begin{array}{l}\text { Salix alba L. } \\
\text { (BCN29777) }\end{array}$ & Sàlix. Saule & Artisanal & Stem & 7 \\
\hline & $\begin{array}{l}\text { Salix fragilis L. } \\
\text { (BCN31305) }\end{array}$ & Vimbera. Vímec. Vimequera. Vim & Artisanal & Stem & 17 \\
\hline Sapindaceae & $\begin{array}{l}\text { Aesculus } \\
\text { hippocastanum L. } \\
\text { (BCN29618) }\end{array}$ & Castanyer bord & Timber & Stem & 1 \\
\hline Saxifragaceae & Bergenia sp. & Hortènsia d'hivern & Ornamental & Whole plant & 1 \\
\hline Smilacaceae & $\begin{array}{l}\text { Smilax aspera L. } \\
\text { (BCN29951) }\end{array}$ & Arítjol & Fuel obtaining & Aerial part & 1 \\
\hline \multirow[t]{2}{*}{ Solanaceae } & $\begin{array}{l}\text { Nicotiana tabacum } \\
\text { L. (BCN48711) }\end{array}$ & Tabac & $\begin{array}{l}\text { Agrosilvopastoral management. } \\
\text { Repellent. Smoking plant }\end{array}$ & Leaf & 3 \\
\hline & $\begin{array}{l}\text { Solanum tuberosum } \\
\text { L. (BCN29797) }\end{array}$ & Patata. Patatera. Trumfera & $\begin{array}{l}\text { Agrosilvopastoral management. } \\
\text { Domestic. Smoking plant }\end{array}$ & $\begin{array}{l}\text { Leaf. Tuber. Whole } \\
\text { plant }\end{array}$ & 4 \\
\hline Typhaceae & $\begin{array}{l}\text { Typha latifolia L. } \\
\text { (BCN31314) }\end{array}$ & Balca & Agrosilvopastoral management. Artisanal & Stem & 10 \\
\hline Ulmaceae & $\begin{array}{l}\text { Ulmus minor Mill. } \\
\text { (BCN113729) }\end{array}$ & Om & $\begin{array}{l}\text { Agrosilvopastoral management. Artisanal. } \\
\text { Timber }\end{array}$ & Stem & 13 \\
\hline Urticaceae & $\begin{array}{l}\text { Urtica dioica L. } \\
\text { (BCN29814) }\end{array}$ & Ortiga & $\begin{array}{l}\text { Agrosilvopastoral management. Folk oral } \\
\text { literature. Not reported }\end{array}$ & $\begin{array}{l}\text { Aerial part. Whole } \\
\text { plant }\end{array}$ & 9 \\
\hline Violaceae & $\begin{array}{l}\text { Viola alba Besser } \\
\text { (BCN27286) }\end{array}$ & Viola. Violeta & Ornamental & Aerial part & 1 \\
\hline Vitaceae & $\begin{array}{l}\text { Vitis vinifera L. } \\
\text { (BCN29972) }\end{array}$ & $\begin{array}{l}\text { Raïm (fruit). Sarment. Vi (elaborated product). Vinagre } \\
\text { (elaborated product). Vinya }\end{array}$ & Not reported & Fruit & 1 \\
\hline
\end{tabular}

confirms the specific epithet of Erica scoparia, which is used, as other Erica species, for broom elaboration. All kind of plant uses are reflected in some vernacular names. We believe that an in-depth research on folk phytonyms (in different areas of a language and in different languages) and of scientific plant names that reflect plant 

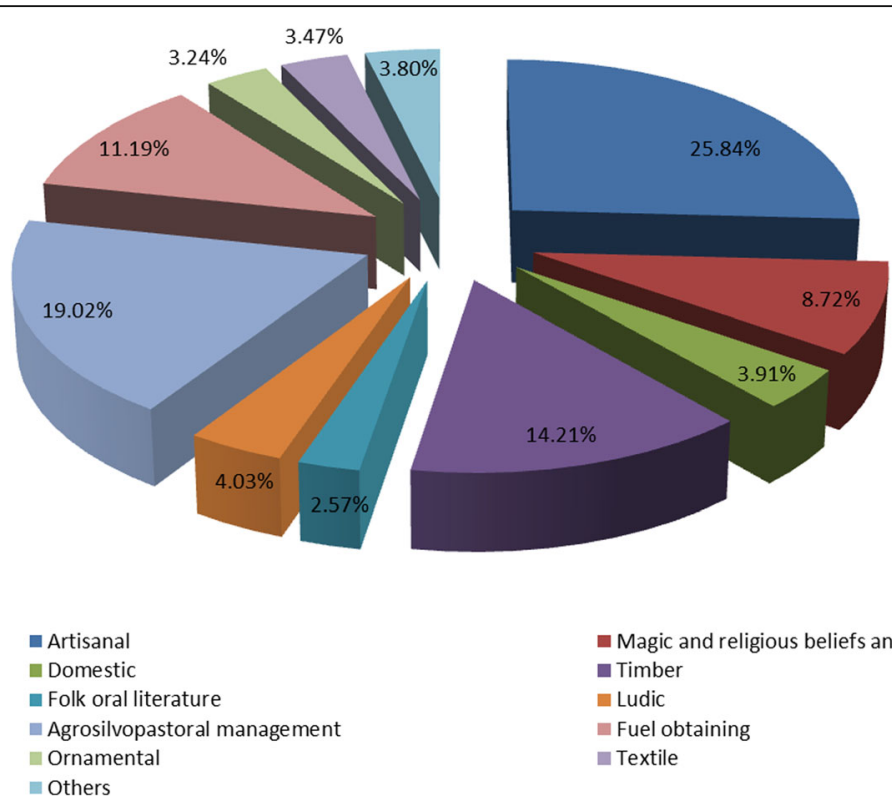

- Others

- Magic and religious beliefs and practices

Fig. 4 Other uses, as a percentage, quoted in Gironès

uses is an interesting field of research, still scarcely or not at all addressed in ethnobotany.

\section{Concluding remarks}

This study has revealed that traditional knowledge is persisting in the studied area if we take into account the numbers of taxa quoted and of use reports, as well as the values of the calculated indexes and despite the proximity to the highly urbanized areas. We have detected a significant number of allochthonous useful plants, and we believe that this subject should be particularly addressed in ethnobotanical studies in other areas throughout the world. The food plant use dataset is particularly important. Conversely, although the knowledge remains in the memory of our informants, the medicinal use of plants is substantially smaller than it used to be (informants often speak in the past of these uses) in their daily life, proving the erosive process in plant traditional knowledge and use that our industrialized societies are experiencing. In this sense, our research helps to alleviate this deterioration and to inventory this heritage, making ready for dissemination and reintroduction to younger generations of the society, who have suffered acculturation, and also for further studies in drug or other useful products development. In any case, even though the current ethnopharmacological pool is eroded and less employed as opposed to some decades ago, as we have shown, the number of medicinal plants and uses recorded are clearly higher than in less industrialized areas, where uses are more persistent. This applies, even more, for the food ethnobotanical corpus and, again to a lesser extent, to the ethnobotany of non-food and non-medicinal plant uses, finally showing the general solidity of ethnobotanical tradition in the area studied, which is now recorded and, thus, protected.

\section{Acknowledgements}

The authors want to thank the informants for sharing with us their knowledge and their time. Samuel Pyke (Botanical Garden of Barcelona) is also acknowledged for his revision of the English language, Josep M. Suñé (Universitat de Barcelona) for his advices regarding pharmaceutical preparations and forms, and Joan Veny (Institut d'Estudis Catalans) for his continuous help in phytonymic questions. This research was supported by projects 2014SGR00514 and 2017SGR1116 from Generalitat de Catalunya (Catalan government) and PRO2017-S02-VALLES from the Institut d'Estudis Catalans. AG benefited from a predoctoral grant of the Universitat de BarceIona (APIF 2015-2018) and a predoctoral contract of project CGL2017-84297$\mathrm{R}$ of the Spanish government. Finally, we acknowledge support of the publication fee by the CSIC Open Access Publication Support Initiative through its Unit of Information Resources for Research (URICI).

\section{Funding}

See acknowledgements

\section{Availability of data and materials Not applicable.}

\section{Authors' contributions}

TG and JV coordinated the study. All authors (AG, GS, JV, and TG) participated in the design of the research, data collection, and results discussion. All authors wrote the manuscript and they read and approved the final manuscript.

\section{Ethics approval and consent to participate}

All the authors agree with the manuscript and consent to participate in it. Concerning the informants, they gave the informed consent (see "Material and Methods" section).

Consent for publication

The authors give their consent for publication of this manuscript. 


\section{Competing interests}

The authors declare that they have no competing interests.

\section{Publisher's Note}

Springer Nature remains neutral with regard to jurisdictional claims in published maps and institutional affiliations.

\section{Author details}

'Laboratori de Botànica (UB) - Unitat associada al CSIC, Facultat de Farmàcia i Ciències de I'Alimentació, Universitat de Barcelona, Avinguda Joan XXIII 27-31, 08028 Barcelona, Catalonia, Spain. 'Institut Botànic de Barcelona (IBB, CSIC-ICUB), Passeig del Migdia s/n, Parc de Montjuïc, 08038 Barcelona, Catalonia, Spain. ${ }^{3}$ Institut de Recerca de la Biodiversitat (IRBio), Universitat de Barcelona, Avinguda Diagonal 643, 08028 Barcelona, Catalonia, Spain. ${ }^{4}$ Secció de Ciències Biològiques, Institut d'Estudis Catalans, Carrer del Carme 47, 08001 Barcelona, Catalonia, Spain.

\section{Received: 31 August 2018 Accepted: 26 February 2019 Published online: 02 April 2019}

\section{References}

1. Mulet L. Aportaciones al conocimiento etnobotánico de la provincia de Castellón: Universitat de València; 1990. PhD thesis

2. Muntané J. Aportació al coneixement de l'etnobotànica de Cerdanya: Universitat de Barcelona; 1991. PhD thesis

3. Bonet MÀ. Estudis etnobotànics a la vall del Tenes (Vallès Oriental): Universitat de Barcelona; 1991. Master thesis

4. Selga A. Estudis etnobotànics a les Guilleries: Universitat de Barcelona; 1998. Master thesis

5. Agelet A. Estudis d'etnobotànica farmacèutica al Pallars: Universitat de Barcelona; 1999. PhD thesis

6. Bonet MÀ. Estudi etnobotànic del Montseny: Universitat de Barcelona; 2001. PhD thesis

7. Muntané J. Etnobotànica, etnofarmàcia i tradicions populars de la Catalunya septentrional (Capcir, Cerdanya i Conflent): Universitat de Barcelona; 2005. PhD thesis

8. Pellicer J. Recerques etnobotàniques al territori diànic o comarques centrals valencianes: Universitat de València; 2005. PhD thesis

9. Rigat M. Estudi etnobotànic de la Vall de Camprodon (Alta Vall del Ter, Pirineus) Universitat de Barcelona; 2005. Master thesis.

10. Parada M. Estudi etnobotànic de l'Alt Empordà: Universitat de Barcelona; 2007. PhD thesis

11. Mayans M. Estudi etnobotànic de Formentera: Universitat de Barcelona; 2013. Master thesis

12. Carrió E. Contribució a l'etnobotànica de Mallorca. La biodiversitat vegetal i la seva gestió en una illa mediterrània: Universitat de Barcelona; 2013. PhD thesis

13. Serrasolses $\mathrm{G}$. Estudis etnobotànics del Gironès occidental: Universitat de Barcelona; 2014. Master thesis

14. Talavera M. La recuperació dels coneixements tradicionals relatius a la biodiversitat com a eina de desenvolupament de nous cultius amb espècies silvestres, i acceptació per part dels consumidors dels productes elaborats amb espècies silvestres i varietats tradicionals. Estudi etnobotànic de la comarca de l'Anoia, desenvolupament de nous cultius amb espècies silvestres, i acceptació per part dels consumidors dels productes elaborats amb espècies silvestres i varietats tradicionals: Universitat de Barcelona; 2018. PhD thesis

15. Ember CR, Ember M. Antropología cultural. 8th ed. Madrid: Prentice Hall; 1997.

16. Tapia GF. Etnobotánica de los amuzgos. Parte 1: Los árboles. Cuadernos de la Casa Chata, 14. México: CISINAH; 1978.

17. Alcorn J. Huastec Mayan ethnobotany. Austin: University of Texas Press; 1984

18. Davis EW. The ethnobotany of chamairo: Mussatia hyacinthina. J Ethnopharmacol. 1983:9:225-36.

19. Schultes RE. Amazonian ethnobotany and the search for new drugs. In: Chadwick DJ, Marsh J, editors. Ethnobotany and the Search for New Drugs (Ciba Foundation Symposium 1185). Chichester: Wiley; 1994.

20. Maffi L, editor. On biocultural diversity: linking language, knowledge and the environment. Washington, D.C.: Smithsonian Institution Press; 2001.
21. Maffi L. Linguistic, cultural, and biological diversity. Ann Rev Ant. 2005;34: 599-617.

22. Sutherland W. Parallel extinction risk and global distribution of languages and species. Nature. 2003:423:276-9.

23. Arnold-Apostolides N. Ethnobotanique et ethnopharmacologie de la flore de Chypre et de l'est méditerranéen (six volumes). Centre Régional de Phytosociologie: Bailleul; 1991.

24. Rivera D, Obón C, Inocencio C, Heinrich M, Verde A, Fajardo J, Llorach R. The ethnobotanical study of local Mediterranean food plants as medicinal resources in southern Spain. J Physiol Pharmacol. 2005;56:97-114.

25. Leonti M, Nebel S, Rivera D. Wild gathered food plants in the European Mediterranean: a comparative analysis. Econ Bot. 2006;60:130-42.

26. Pieroni A. Local plant resources in the ethnobotany of Theth, a village in the northern Albanian Alps. Genet Resour Crop Evol. 2008;55:1197.

27. Pardo-de-Santayana M, Pieroni A, Puri RK, editors. Ethnobotany in the new Europe. People, health and wild plant resources. Environmental anthropology and ethnobiology (vol. 14). New York-Oxford: Berghahn Books; 2013.

28. Harshberger JW. Purposes of ethnobotany. Bot Gazet. 1986;21:146-54.

29. Neves JM, Matos C, Mountinho C, Queiroz G, Rebelo L. Ethnopharmacological notes about ancient uses of medicinal plants in TrásOs-Montes (northern of Portugal). J Ethnopharmacol. 2009;124:270-83.

30. Benítez G, González-Tejero MR, Molero-Mesa J. Pharmaceutical ethnobotany in the western part of Granada province (southern Spain): Ethnopharmacological synthesis. J Ethnopharmacol. 2010;129(1):87-105

31. Yesilada E. Ethnopharmacology in the Eastern Mediterranean and the Middle East: 'The Sun Rises from the East, but Shines on the Eastern Mediterranean'. In: Heinrich M, Jäger AK, editors. Ethnopharmacology. Postgraduate pharmacy series. Chichester: Wiley; 2015.

32. Tardío J, Pardo-de-Santayana M, Morales R. Ethnobotanical review of wild edible plants in Spain. Bot J Linn Soc. 2006;152:27-71.

33. Pardo-de-Santayana M, Tardío J, Blanco E, Carvalho AM, Lastra JJ, San Miguel E, Morales R. Traditional knowledge of wild edible plants used in the northwest of the Iberian Peninsula (Spain and Portugal): a comparative study. J Ethnobiol Ethnomed. 2007;3(1):27.

34. Parada M, Carrió E, Vallès J. Ethnobotany of food plants in the alt Emporda region (Catalonia, Iberian Peninsula). J App Bot Food Qual. 2011:84:11-25.

35. Łuczaj L. Ethnobotanical review of wild edible plants of Slovakia. Acta Soc Bot Pol. 2012:81:245-55.

36. Joshi N, Siwakoti M, Kehlenbeck K. Wild vegetable species in Makawanpur District, Central Nepal: developing a priority setting approach for domestication to improve food security. Econ Bot. 2015;69(2):161-70.

37. Gras A, Garnatje T, Bonet MÀ, Carrió E, Mayans M, Parada M, Rigat M, Vallès J. Beyond food and medicine, but necessary for life, too: other folk plant uses in several territories of Catalonia and the Balearic Islands. J Ethnobiol Ethnomed. 2016;12:23.

38. IDESCAT. Institut d'Estadística de Catalunya. http://www.idescat.cat. Accessed 15 Aug 2018.

39. Servei Meteorològic de Catalunya, Generalitat de Catalunya. www.meteo.cat. Accessed 15 Aug 2018.

40. Girbal J. Flora i vegetació del Gironès. Universitat Autònoma de Barcelona; 1984. PhD thesis.

41. Pujadas JJ, Comas D, Roca J. Etnografia. Barcelona: Universitat Oberta de Catalunya; 2004

42. Cook FEM. Economic botany data collection standard. Kew: Royal Botanic Gardens; 1995.

43. de Bolòs O, Vigo J. Flora dels Països Catalans (four volumes). Barcelona: Editorial Pòrtic; 1984-2001.

44. de Bolòs O, Vigo J, Masalles R, Ninot J. Flora manual dels Països Catalans. 3rd ed. Editorial Pòrtic: Barcelona; 2005

45. Angiosperm Phylogeny Group. An update of the Angiosperm Phylogeny Group classification for the orders and families of flowering plants: APG IV. Bot J Linn Soc. 2016;181(1):1-20.

46. International Society of Ethnobiology. International Society of Ethnobiology Code of Ethics (with 2008 additions). http://ethnobiology.net/code-of-ethics. Accessed 15 Aug 2018.

47. Rosenthal JP. Politics, culture, and governance in the development of prior informed consent in indigenous communities. Curr Anthropol. 2006;47(1):119-42.

48. Vandebroek I, Thomas E, Sanca S, Van Damme P, Van Puyelde L, De Kimpe N. Comparison of health conditions treated with traditional and biomedical health care in a Quechua community in rural Bolivia. J Ethnobiol Ethnomed. 2008;4:1. 
49. Portères R. Cours d'Ethno-botanique et Ethno-zoologie (1969-1970). Volume I, Ethno-botanique générale. Paris: Muséum National d'Histoire Naturelle (Laboratoire d'Ethno-botanique et Ethno-zoologie), Faculté des Lettres (Institut d'Ethnologie); 1970.

50. Trotter RT, Logan MH. Informant consensus: a new approach for identifying potentially effective medicinal plants. In: Etkin NL, editor. Plants in indigenous medicine and diet, Behavioural approaches. Bredford Hills: Redgrave Publishing Company; 1986. p. 91-112.

51. Bonet MA, Vallès J. Plantes, remeis i cultura popular del Montseny. Etnobotànica d'una Reserva de la Biosfera. Figueres-Granollers: Brau Edicions i Museu de Granollers Ciéncies Naturals; 2006

52. Gras A, Parada M, Rigat M, Vallès J, Garnatje T. Folk medicinal plant mixtures: establishing a protocol for further studies. J Ethnopharmacol. 2018;214:244-73.

53. Bonet MÀ, Parada M, Selga A, Vallès J. Studies on pharmaceutical ethnobotany in the regions of l'Alt Empordà and les Guilleries (Catalonia, Iberian Peninsula). J Ethnopharmacol. 1999;68:145-68.

54. Johns T, Kokwaro JO, Kimanani EK. Herbal remedies of the Luo of Siaya district, Kenya: establishing quantitative criteria for consensus. Econ Bot. 1990;44:369-81.

55. Bonet MÀ, Vallès J. Pharmaceutical ethnobotany in the Montseny biosphere reserve (Catalonia, Iberian Peninsula). General results and new or rarely reported medicinal plants. J Pharm Pharmacol. 2003:55:259-70.

56. Parada M, Bonet MÀ, Carrió E, Vallès J. Ethnobotany of the alt Empordà region (Catalonia, Iberian Peninsula). Plants used in human traditional medicine. J Ethnopharmacol. 2009;124:609-18.

57. Carrió E, Vallès J. Ethnobotany of medicinal plants used in eastern Mallorca (Balearic Islands, Mediterranean Sea). J Ethnopharmacol. 2012;141:1021-104.

58. Rigat M, Gras A, Vallès J, Garnatje T. Estudis etnobotànics a la comarca del Ripollès (Pirineu, Catalunya, pensínsula Ibèrica). Collect Bot. 2017;36:e003.

59. Rigat M, Bonet MÀ, Garcia S, Garnatje T, Valles J. Ethnobotanical studies in the high river Ter valley (Pyrenees, Catalonia, Iberian Peninsula). Non-crop food vascular plants and crop food plants with medicinal properties. Ecol Food Nutr. 2009;48:303-26

60. Serrasolses G, Calvet-Mir L, Carrió E, D’Ambrosio U, Garnatje T, Parada M, Vallès J, Reyes-Garcia V. A matter of taste: local explanations for the consumption of wild food plants in the Catalan Pyrenees and the Balearic Islands. Econ Bot. 2016;70:176-89.

61. Heinrich M, Ankli A, Frei B, Weimann C, Sticher O. Medicinal plants in Mexico: healers' consensus and cultural importance. Soc Sci Med. 1998;47:1857-9.

62. Leonti M, Vibrans H, Sticher O, Heinrich M. Ethnopharmacology of the Popoluca, Mexico: an evaluation. J Pharm Pharmacol. 2001;53:1653-9.

63. Esakkimuthu S, Sylvester S, Mutheeswaran S, Paulraj M, Pandikumar $P$, Ignacimuthu S, Al-Dhabi NA. A study on food-medicine contínuum among the non-institutionally trained siddha practitioners of Tiruvallur district, Tamil Nadu, India. J Ethnobiol Ethnomed. 2018;14:45.

64. Axiotis E, Halabalaki M, Skaltsounis LA. An ethnobotanical study of medicinal plants in the Greek islands of North Aegean region. Front Pharmacol. 2018;9:409.

65. Akgul A, Akgul A, Senol SG, Yildirim H, Secmen O, Dogan Y. An ethnobotanical study in Midyat (Turkey), a city on the silk road where cultures meet. J Ethnobiol Ethnomed. 2018;14:12.

66. Ong HG, Ling SM, Win TTM, Kang DH, Kim YD. Ethnomedicinal plants and traditional knowledge among three Chin indigenous groups in Natma Taung National Park (Myanmar). J Ethnopharmacol. 2018;225:136-58.

67. Preston CD, Pearman DA, Hall AR. Archaeophytes in Britain. Bot J Linn Soc. 2004;145:257-94

68. Agelet A, Vallès J. Studies on pharmaceutical ethnobotany in the region of Pallars (Pyrenees, Catalonia, Iberian Peninsula). Part I. General results and new or very rare medicinal plants. J Ethnopharmacol. 2001;77:57-70.

69. González JA, García-Barriuso M, Amich F. Ethnobotanical study of medicinal plants traditionally used in the Arribes del Duero, western Spain. J Ethnopharmacol. 2010;131:343-55.

70. Chadwick DJ, Marsh J, editors. Ethnobotany and the Search for New Drugs (Ciba Foundation Symposium 1185). Chichester: Wiley; 1994.

71. Tu Y. Artemisinin — a gift from traditional Chinese medicine to the world (Nobel lecture). Angew Chem Int Ed. 2016;55:10210-26.

72. Ali-Stayeh MS, Yaniv Z, Mahajna J. Ethnobotanical survey in the Palestinian area: a classification of the healing potential of medicinal plants. J Ethnopharmacol. 2000;73:221-32.

73. Pieroni A. Medicinal plants and food medicines in the folk traditions of the upper Lucca Province, Italy. J Ethnopharmacol. 2000;70:235-73.
74. Maggini R, Benvenuti S, Leoni F, Pardossi A. Terracrepolo (Reichardia picroides (L.) Roth.): wild food or new horticultural crop? Sci Hortic. 2018;204:224-31.

75. Akerreta S. Etnobotánica farmacéutica en Navarra: del uso tradicional de las plantas medicinales a su evidencia científica: Universidad de Navarra; 2009. $\mathrm{PhD}$ thesis

76. Raja D. Estudis etnobotànics a la comarca de la Segarra. MSc thesis: Universitat de Barcelona; 1995.

\section{Ready to submit your research? Choose BMC and benefit from:}

- fast, convenient online submission

- thorough peer review by experienced researchers in your field

- rapid publication on acceptance

- support for research data, including large and complex data types

- gold Open Access which fosters wider collaboration and increased citations

- maximum visibility for your research: over $100 \mathrm{M}$ website views per year

At BMC, research is always in progress.

Learn more biomedcentral.com/submissions 